\title{
Equivariant Quantum Cohomology of the Odd Symplectic Grassmannian
}

\author{
Ryan M. Shifler \\ Dissertation submitted to the Faculty of the \\ Virginia Polytechnic Institute and State University \\ in partial fulfillment of the requirements for the degree of
}

Doctor of Philosophy

in

Mathematics
C. Leonardo Mihalcea, Chair
Mark M. Shimozono
Daniel D. Orr
Peter E. Haskell

March 21, 2017

Blacksburg, Virginia

Keywords: odd symplectic, quantum cohomology, Chevalley formula Copyright 2017, Ryan M. Shifler 


\section{Equivariant Quantum Cohomology of the Odd Symplectic Grassmannian}

Ryan M. Shifler

\section{ABSTRACT}

The odd symplectic Grassmannian IG $:=\operatorname{IG}(k, 2 n+1)$ parametrizes $k$ dimensional subspaces of $\mathbb{C}^{2 n+1}$ which are isotropic with respect to a general (necessarily degenerate) symplectic form. The odd symplectic group acts on IG with two orbits, and IG is itself a smooth Schubert variety in the submaximal isotropic Grassmannian $\mathrm{IG}(k, 2 n+2)$. We use the technique of curve neighborhoods to prove a Chevalley formula in the equivariant quantum cohomology of IG, i.e. a formula to multiply a Schubert class by the Schubert divisor class. This generalizes a formula of Pech in the case $k=2$, and it gives an algorithm to calculate any quantum multiplication in the equivariant quantum cohomology ring. 


\section{Equivariant Quantum Cohomology of the Odd Symplectic Grassmannian}

Ryan M. Shifler

\section{GENERAL AUDIENCE ABSTRACT}

The thesis studies a problem in the general area of Combinatorial Algebraic Geometry. The goal of Algebraic Geometry is to study solutions to systems to polynomial equations. Such systems are ubiquitous in scientific research. We study a problem in enumerative geometry on a space called the odd symplectic Grassmannian. The problem seeks to find the number of curves which are incident to certain subspaces of the given Grassmannian. Due to subtle geometric considerations, the count is sometimes virtual, meaning that some curves need to be counted negatively. The rigorous context of such questions is that of Gromov-Witten theory, a subject with roots in physics. Our space affords a large number of symmetries, and the given counting problems translate into significant amount of combinatorial manipulations. The main result in the dissertation is a combinatorial algorithm to perform the virtual curve counting in the odd-symplectic Grassmannian. 


\section{Dedication}

To my parents 


\section{Acknowledgments}

First and foremost I thank my advisor, Leonardo Mihalcea, for investing his time and effort into my graduate school career. He introduced me to a project that is both intriguing and challenging. I also thank Bud Brown, Anders Buch, Dan Orr, and Mark Shimozono for many useful conversations. I also had many useful conversations with Kelli Karcher, Greg Marx, Tanner Slagel, Andy Wills, and Camron Withrow. Then there is Samantha Erwin who was a positive constant during my time at Virginia Tech. We have been on this journey from day one through Professor Ball's Real Analysis class, through prelims and research, to our defense dates that are one day apart.

I'd thank Mike Bardzell, Christopher Lewis, Kurt Ludwick, Amy Matonak, Kathleen Shannon, Don Spickler, and Katie Walsh for keeping me on a mathematical trajectory. I thank Robin Papa for putting me on this path back in high school.

I thank the many running friends have made over the years that have supported my endeavors. In particular I'd like to name Jaime Dick, Brian Ferrari, and Jaime Mason as particularly influential.

Finally a special thanks to my parents and family who supported me during my time in graduate school. 


\section{Contents}

1 Introduction $\quad 1$

1.1 Motivation . . . . . . . . . . . . . . . . . . 1

1.2 Statement of results . . . . . . . . . . . . . . . . . . 3

1.2.1 Odd symplectic Grassmannian . . . . . . . . . . . . . . 3

2 Preliminaries $\quad 12$

2.1 The odd symplectic group . . . . . . . . . . . . . . . . . . . . 12

2.2 The odd symplectic flag varieties . . . . . . . . . . . . . . . 13

2.3 The Weyl group and odd-symplectic minimal representatives . . . . . 14

2.3.1 Type A and Type C Bruhat order compatibility . . . . . . . . 16

2.3.2 Hecke Product . . . . . . . . . . . . . . . . . . . . 17

2.4 Schubert Varieties in even and odd flag manifolds . . . . . . . . . 18

3 (Equivariant) cohomology 20

4 (Equivariant) Quantum cohomology 22

4.1 Equivariant Gromov-Witten invariants . . . . . . . . . . . . . . . . . 22

4.2 The (equivariant) quantum cohomology ring . . . . . . . . . . . 24

5 The moment graphs of even and odd symplectic flag manifolds 26

5.1 The Grassmannian case . . . . . . . . . . . . . . . 27

6 Curve neighborhoods $\quad 29$

6.1 Curve neighborhoods of Schubert varieties in $\mathrm{IG}^{\text {even }} \ldots . . . . . . .29$

6.2 Curve neighborhoods for IG. . . . . . . . . . . . . . . . . . 31

7 Vanishing of Chevalley Gromov-Witten invariants 34

8 Lines in IG 35

8.1 Lines intersecting the open orbit $X^{\circ} \ldots \ldots \ldots$

8.2 Lines in the closed orbit . . . . . . . . . . . . . . . . 39

8.3 Lines with two marked points . . . . . . . . . . . . . . 41

9 Line neighborhoods $\quad 43$ 
11 Equivariant quantum Chevalley rule with $(n-k)$-strict partitions 49

11.1 A dictionary permutations - partitions . . . . . . . . . . . . 49

11.2 The equivariant quantum Chevalley formula . . . . . . . . . . . . . 52

11.2.1 The EQ Chevalley formula for $\operatorname{IG}(3,7) \ldots \ldots 55$

12 Calculating $\mathrm{QH}_{T}^{*}(\mathrm{IG}) \quad 56$

12.1 Notation . . . . . . . . . . . . . . . . . . . . . 57

12.2 Two formulae . . . . . . . . . . . . . . . . . . . . . . . . . . . . . . . . . . . 58

12.3 An algorithm to compute the EQ coefficients . . . . . . . . . . . . 60

12.4 Multiplication table for $\mathrm{QH}_{T}^{*}(\mathrm{IG}(2,5)) \ldots \ldots \ldots$. . . . . . . 62

A The first Chern class of the tangent bundle $c_{1}\left(T_{\mathrm{IG}}\right) . \quad 64$

$\begin{array}{ll}\text { B Lines } & 71\end{array}$ 


\section{Chapter 1}

\section{Introduction}

\subsection{Motivation}

The goal of Schubert Calculus and Enumerative Geometry is to count solutions to geometric questions. Consider a finite number of "general" points in a plane (rigorously this example is in the complex projective plane $\left.\mathbb{P}^{2}\right)$. The word "general" roughly means we avoid special cases (e.g. 3 points are not general in the plane if a line can be drawn through all of them). How many lines contain $n$ general points in the plane? The answer is either zero, finite, or infinite. So what does $n$ need to be so the answer is nonzero and finite? It is easy to see $n=2$ and the number lines through 2 general points is 1 . Less specifically, how many rational curves of degree $d$ contain $n$ general points in the plane? The answer is nonzero and finite when $n=3 d-1$. The number of rational curves of degree $d$ containing $3 d-1$ general points in the plane is $N_{d}$ (see [21] for details) where $N_{1}=1$ and

$$
N_{d}=\sum_{d_{1}+d_{2}=d_{,}, d_{1}, d_{2}>0} N_{d_{1}} N_{d_{2}}\left(d_{1}^{2} d_{2}^{2}\left(\begin{array}{c}
3 d-4 \\
3 d_{1}-2
\end{array}\right)-d_{1}^{3} d_{2}\left(\begin{array}{c}
3 d-4 \\
3 d_{1}-1
\end{array}\right)\right)
$$

The answer for $d$ equal to 1, 2, and 3 has been known for a significant period of time. The answer for $d=4$ was found by Zeuthen in 1873. The question for $d \geq 5$ remained open until 1993 when Kontsevich solved the problem by studying the (big) quantum cohomology of $\mathbb{P}^{2}$. The reference [1, page 15] contains further details.

In 1874 Hermann Schubert published the book Kalkül der Abzählenden Geometrie (Calculus of Enumerative Geometry [60]) which was about counting the number of geometric objects such as points, lines, and planes that satisfy certain conditions. Schubert's methods of enumerative geometry did not rest on a solid rigorous foundation. The 15th Hilbert problem is to remedy this issue. It states: "The problem consists in this: To establish rigorously and with an exact determination of the limits of their validity those geometrical numbers which Schubert especially has determined on the basis of the so-called principle of special position, or conservation of number, by means of the enumerative calculus developed by him." The problem was solved 
with Intersection Theory (see [24]). The goal of counting geometric objects is applied to more general geometric spaces such as the Grassmannian and flag varieties.

Let $\operatorname{Gr}(k, n)$ denote the Grassmannian which is the space of $k$ dimensional vector spaces in $\mathbb{C}^{n}$. Let $\mathrm{Fl}(n)$ denote the flag variety which is the space of sequences of vectors spaces $\left(V_{1} \subset V_{2} \subset \cdots \subset V_{n-1} \subset \mathbb{C}^{n}\right)$ with $\operatorname{dim} V_{i}=i$. The spaces $\operatorname{Gr}(k, n)$ and $\operatorname{Fl}(n)$ are special cases of homogeneous spaces $G / P$ where $G$ is a semisimple complex Lie group and $P$ is a parabolic subgroup. Special subspaces are Schubert varieties which parameterize flags intersecting a given flag in prescribed dimensions. See Brion's lecture notes [7] for details. The classes, $\sigma(\lambda)$ where $\lambda$ varies in an appropriate indexing set, of the Schubert varieties of $\operatorname{Gr}(k, n)$ and $\operatorname{Fl}(n)$ form a basis for the cohomology rings $\mathrm{H}^{*}(\mathrm{Gr}(k, n))$ and $\mathrm{H}^{*}(\mathrm{Fl}(n))$, respectively. The goal of Schubert calculus is to calculate the structure constants that are the coefficients in the multiplication

$$
\sigma(\lambda) \cdot \sigma(\mu)=\sum c_{\lambda, \mu}^{\nu} \sigma(\nu)
$$

where $\sigma(\lambda)$ is the class of the Schubert variety indexed by $\lambda$. The coefficient $c_{\lambda, \mu}^{\nu}$ counts the number of intersection points of general translates of the corresponding Schubert varieties. A Littlewood-Richardson (LR) rule, which is a positive combinatorial formula, is known for calculating the coefficients in $\mathrm{H}^{*}(\operatorname{Gr}(k, n))[23,37]$. However, a LR rule for the coefficients in $\mathrm{H}^{*}(\mathrm{Fl}(n))$ is still unknown.

The ring $\mathrm{QH}^{*}(X)$ is the quantum cohomology ring of a sufficiently nice space $X$. We are interested in calculating the structure constants that are the coefficients in the multiplication

$$
\sigma(\lambda) \star \sigma(\mu)=\sum c_{\lambda, \mu}^{\nu, d} q^{d} \sigma(\nu)
$$

where $\sigma(\lambda)$ is the class of the Schubert variety indexed by $\lambda$ and $q$ is the quantum parameter. The structure constants of $\mathrm{QH}^{*}(X)$ are three point Gromov-Witten invariants and count the number of degree $d$ rational curves that intersect the general translates of three Schubert varieties when $X$ is homogeneous. A LR that uses puzzles is proved by Buch, Kresch, Purbhoo, and Tamvakis in [12] for $X=\operatorname{Gr}(k, n)$. The Gromov-Witten invariants are said to be enumerative since they are counting rational curves. For a general space $X$ the Gromov-Witten invariants may not be enumerative and calculating three point Gromov-Witten invariants may be difficult. The quantum cohomology rings $\mathrm{QH}^{*}(X)$ are mostly unknown. The quantum cohomology of the Grassmannian, flag, and partial flags were studied in $[3,10,11,17,49-51]$. The equivariant quantum cohomology ring $\mathrm{QH}_{T}^{*}(X)$, where $T$ is torus, is often better to consider since there is additional structure. The equivariant version of quantum cohomology was introduced by Givental and Kim as an additional tool to understand quantum cohomology $[27,28,35]$.

For this project we study the quantum and equivariant quantum cohomology rings of a particular non-homogeneous space. The space is the "odd symplectic Grassmannian" denoted by $\operatorname{IG}(k, 2 n+1)$ which has the action of the odd symplectic group $\mathrm{Sp}_{2 n+1}$. The space is the parameterization of $k$ dimensional vector spaces in $\mathbb{C}^{2 n+1}$ that preserves a general antisymmetric (necessarily degenerate) bilinear form. The 
space is a smooth Schubert variety in the usual symplectic Grassmannian IG $(k, 2 n+2)$ where the form is non-degenerate. It has two orbits. One open and the other closed. Since the space has a finite number of orbits we can expect it to have properties that are similar to those that occur in the homogeneous case. Since the odd symplectic Grassmannian lives just outside of the homogeneous case the calculation of the equivariant quantum cohomology ring $\mathrm{QH}_{T}^{*}(\mathrm{IG}(k, 2 n+1))$ where $T$ is a maximal torus of $\mathrm{Sp}_{2 n+1}$ is a natural next step. This calculation is the main focus of this dissertation.

The odd symplectic Grassmannian is related to the orthogonal Grassmannian, the Lagrangian Grassmannian, and the isotropic Grassmannian which are homogeneous spaces. The classical cohomology and quantum cohomology of these spaces were study by Buch, Kresch, and Tamvakis in [14,41,42]; by Tamvakis in [62]; by Bergeron and Sottile in [4]; by Bertram in [5]; and by Sottile in [61].

The equivariant quantum Chevalley formula is the multiplication of a Schubert class by the divisor class written as a sum of Schubert classes of $\mathrm{QH}_{T}^{*}(\mathrm{IG}(k, 2 n+1))$. Historically, the Chevalley formula has been used to calculate the equivariant quantum cohomology rings. Okounkov presented some of the ideas in [54] along with Molev and Sagan in [52]. These calculations have been used by Knutson and Tao in the equivariant case in [37]; Mihalcea in the equivariant quantum case in [50,51]; and by Buch, Chaput, Mihalcea, and Perrin in the equivariant quantum $K$-theory of cominuscule varieties in [8]. Pech is able to use a Kleiman-Bertini type lemma proved by Graber in [30] to show that some coefficients contain enumerative data and proves a (nonequivariant) Pieri formula for $\operatorname{IG}(2,2 n+1)$. In joint work with Mihalcea we use the technique of curve neighborhoods to prove an equivariant quantum Chevalley formula for $\operatorname{IG}(k, 2 n+1)$.

\subsection{Statement of results}

\subsubsection{Odd symplectic Grassmannian}

Let $E$ be a complex vector space of dimension $2 n+1$. Let $\omega: E \otimes E \rightarrow \mathbb{C}$ be a general antisymmetric bilinear form. This is equivalent to $\operatorname{dim} \operatorname{ker} \omega=1$. The form $\omega$ restricts to a $2 n$ dimensional space $F$ where $E=\operatorname{ker} \omega \oplus F$.

The odd symplectic group is the subgroup of $\mathrm{GL}(E)$ which preserves this symplectic form:

$$
\operatorname{Sp}_{2 n+1}(E)=\{g \in \mathrm{GL}(E): \omega(g . u, g . v)=\omega(u, v), \forall u, v \in E\} .
$$

The odd symplectic group was introduced by Proctor in $[57,58]$. The elements of the odd symplectic group $\operatorname{Sp}_{2 n+1}(E)$ are matrices of the form

$$
\operatorname{Sp}_{2 n+1}(E)=\left\{\left(\begin{array}{cc}
\lambda & a \\
0 & S
\end{array}\right): \lambda \in \mathbb{C}^{*}, a \in \mathbb{C}^{2 n}, S \in \operatorname{Sp}_{2 n}(F)\right\}
$$

where $\operatorname{Sp}_{2 n}(F)$ is the usual symplectic group. The set $B \subset \operatorname{Sp}_{2 n+1}(E)$ of upper triangular matrices is a Borel subgroup and the set $T \subset \operatorname{Sp}_{2 n+1}(E)$ of diagonal matrices 
is a maximal torus subgroup. The odd symplectic Grassmannian is

$$
\operatorname{IG}(k, 2 n+1)=\{V \subset E: \operatorname{dim} V=k, \omega(u, v)=0 \forall u, v \in V\} .
$$

The space $\operatorname{IG}(k, 2 n+1)$ is a smooth subvariety of codimension $\frac{k(k-1)}{2}$ in $\operatorname{Gr}(k, 2 n+1)=$ $\left\{V \subset \mathbb{C}^{2 n+1}: \operatorname{dim} V=k\right\}$. If $k=n+1$ then $\operatorname{IG}(n+1,2 n+1)$ is isomorphic to the Lagrangian Grassmannian $\operatorname{IG}(n, 2 n)$ and if $k=1$ then $\operatorname{IG}(1,2 n+1)$ is isomorphic to $\mathbb{P}^{2 n}$.

Proposition 1.2.1. For $1<k<n+1$ the odd symplectic group $\operatorname{Sp}_{2 n+1}(E)$ acts on the odd symplectic Grassmannian $\mathrm{IG}(k, 2 n+1)$ with two orbits:

$$
\begin{aligned}
& X_{c}=\{V \in \operatorname{IG}(k, 2 n+1): \operatorname{ker} \omega \in V\} \text { the closed orbit, } \\
& X^{\circ}=\{V \in \operatorname{IG}(k, 2 n+1): \operatorname{ker} \omega \notin V\} \text { the open orbit. }
\end{aligned}
$$

The closed orbit $X_{c}$ is isomorphic to $\mathrm{IG}(k-1,2 n)$.

The space IG $(k, 2 n+1)$ can be viewed as an intermediate symplectic Grassmannian since the closed orbit is isomorphic to $\operatorname{IG}(k-1,2 n)$. That is we have the natural embeddings

$$
\mathrm{IG}(k-1,2 n) \hookrightarrow \mathrm{IG}(k, 2 n+1) \hookrightarrow \mathrm{IG}(k, 2 n+2) .
$$

The space $\operatorname{IG}(k, 2 n+1)$ has an associated combinatorial structure that is derived from the combinatorics of $\operatorname{IG}(k, 2 n+2)$. Fix an ordering

$$
1<2<3<\cdots<n+1<\overline{n+1}<\bar{n}<\cdots<\overline{2}<\overline{1} .
$$

The elements of the hyperoctahedral group will be used to define Schubert varieties. The hyperoctahedral group is $W=\left\{w \in S_{2 n+2}: w(\bar{i})=\overline{w(i)}\right\}$ where $S_{2 n+2}$ is the symmetric group acting on the $2 n+2$ elements in the set $\{1,2,3, \cdots, n+$ $1, \overline{n+1}, \bar{n}, \cdots, \overline{2}, \overline{1}\}$. The set of minimal length representatives $W^{P_{k}} \subset W$ that correspond to $\mathrm{IG}(k, 2 n+2)$ are

$$
(w(1)<w(2)<\cdots<w(k))=w(1) w(2) \cdots w(k) \mid w(k+1) \cdots w(n+1)
$$

where $w(k+1)<\cdots<w(n+1)$ and each $w(i)$ is bar free (i.e. $w(n+1) \leq n+1)$ for $k+1 \leq i \leq n+1$. The odd symplectic permutations for $\operatorname{IG}(k, 2 n+1)$ are the elements of the set

$$
W^{2 n+1}=\left\{w \in W^{P_{k}}: w(k)<\overline{1}\right\} .
$$

Example 1.2.2. Consider $n=7$ and $k=5$. Then

$$
(3<5<8<\overline{4}<\overline{2})=358 \overline{4} \overline{2} \mid 167 \in W^{2 n+1}
$$

and

$$
(3<5<8<\overline{4}<\overline{1})=358 \overline{4} \overline{1} \mid 267 \notin W^{2 n+1} .
$$


A partition $\left(\lambda_{1} \geq \lambda_{2} \geq \cdots \geq \lambda_{k}\right)$ is $(n-k)$-strict if $\lambda_{j}>n-k$ implies $\lambda_{j}>\lambda_{j+1}$. Let the set $\Lambda$ consist of $(n-k)$-strict partitions $\left(2 n+1-k \geq \lambda_{1} \geq \cdots \geq \lambda_{k} \geq-1\right)$ such that if $\lambda_{k}=-1$ then $\lambda_{1}=2 n+1-k$. This is Pech's slight modification of notation used by Buch, Kresch, and Tamvakis in [15]. The elements of $\Lambda$ are in bijection with elements of $W^{2 n+1}$.

Let $B_{2 n+2}$ be the Borel subgroup consisting of upper triangular matrices in the usual symplectic group $\mathrm{Sp}_{2 n+2}$. Let $T_{2 n+2}$ be the maximal torus subgroup consisting of diagonal matrices in the usual symplectic group $\mathrm{Sp}_{2 n+2}$. The set of $T$-fixed points of $\operatorname{IG}(k, 2 n+1)$ equals the set of $T_{2 n+2}$-fixed points of $\operatorname{IG}(k, 2 n+1)$. The $B$ orbits and $B_{2 n+2}$ orbits are equal so the Schubert cells of $\operatorname{IG}(k, 2 n+1)$ are

$$
C_{w}=B w P=B_{2 n+2} w P_{2 n+2}
$$

where $w$ is a $T$-fixed point. Thus, the natural embedding $\iota: \operatorname{IG}(k, 2 n+1) \hookrightarrow$ $\mathrm{IG}(k, 2 n+2)$ identifies $\mathrm{IG}(k, 2 n+1)$ with a smooth Schubert variety of $\mathrm{IG}(k, 2 n+2)$ and the Schubert varieties of $\mathrm{IG}(k, 2 n+1)$ are Schubert varieties of $\operatorname{IG}(k, 2 n+2)$ contained in $\operatorname{IG}(k, 2 n+1)$. The Schubert variety corresponding to $w$ will be denoted by $X(w)$ for all $w \in W^{2 n+1}$ (or Schubert variety corresponding to $\lambda$ will be denoted by $X(\lambda)$ for all $\lambda \in \Lambda)$.

Let $X=\mathrm{IG}(k, 2 n+1)$. Let $\overline{\mathcal{M}}_{0, r}(\mathrm{IG}(k, 2 n+1), d)$ be the Kontsevich moduli space of stable maps of degree $d$ to $\operatorname{IG}(k, 2 n+1)$ with $r$ marked points $(r \geq 0)$ equipped with evaluations maps $\mathrm{ev}_{i}: \overline{\mathcal{M}}_{0, r}(\mathrm{IG}(k, 2 n+1), d) \rightarrow \operatorname{IG}(k, 2 n+1)$; see e.g. [21]. This is a projective algebraic variety of expected dimension

$\operatorname{expdim} \overline{\mathcal{M}}_{0, r}(\mathrm{IG}(k, 2 n+1), d)=\operatorname{dim} \mathrm{IG}(k, 2 n+1)+d \cdot(2 n+2-k)+r-3$.

Let $\gamma_{1}, \ldots, \gamma_{r} \in \mathrm{H}^{*}(\mathrm{IG}(k, 2 n+1))$ and $d \in H_{2}(\mathrm{IG}(k, 2 n+1))$ be an effective degree. The $r$-point, genus $0, \mathrm{GW}$ invariant is defined by

$$
\left\langle\gamma_{1}, \ldots, \gamma_{r}\right\rangle_{d}:=\int_{\left[\overline{\mathcal{M}}_{0, r}(\mathrm{IG}(k, 2 n+1), d)\right]^{v i r}} \operatorname{ev}_{1}^{*}\left(\gamma_{1}\right) \cup \operatorname{ev}_{2}^{*}\left(\gamma_{2}\right) \cup \ldots \cup \operatorname{ev}_{r}^{*}\left(\gamma_{r}\right),
$$

where $\left[\overline{\mathcal{M}}_{0, r}(\operatorname{IG}(k, 2 n+1), d)\right]^{\text {vir }}$ is the virtual fundamental class. This is a cycle

$$
\left[\overline{\mathcal{M}}_{0, r}(\mathrm{IG}(k, 2 n+1), d)\right]^{\text {vir }} \in H_{2 \operatorname{expdim}} \overline{\mathcal{M}}_{0, r}(\mathrm{IG}(k, 2 n+1), d)\left(\overline{\mathcal{M}}_{0, r}(\mathrm{IG}(k, 2 n+1), d)\right) .
$$

The integral means to take the degree 0 part after capping $\operatorname{ev}_{1}^{*}\left(\gamma_{1}\right) \cup \operatorname{ev}_{2}^{*}\left(\gamma_{2}\right) \cup \ldots \cup$ $\operatorname{ev}_{r}^{*}\left(\gamma_{r}\right)$ with $\left[\overline{\mathcal{M}}_{0, r}(\mathrm{IG}(k, 2 n+1), d)\right]^{\text {vir }}$.

The $T$-equivariant quantum cohomology ring $\mathrm{QH}_{T}^{*}(\mathrm{IG}(k, 2 n+1))$ is a graded algebra over the polynomial ring $\mathrm{H}_{T}^{*}(p t)[q]$. The degree of $q$ is $2 n+2-k$ and the classes of Schubert varieties $\{[X(u)]\}_{u \in W^{2 n+1}}$ form an additive basis. The multiplicative structure of $\mathrm{QH}_{T}^{*}(\mathrm{IG}(k, 2 n+1))$ is given by

$$
[X(u)] \star[X(v)]=\sum_{w, d} c_{u, v}^{w, d} q^{d}[X(w)]
$$


where $c_{u, v}^{w, d}=\left\langle[X(u)],[X(v)],[X(w)]^{\vee}\right\rangle_{d}$.

If $X$ were a homogeneous space then $c_{u, v}^{w, d}$ would count rational curves through translates of $X(u), X(v)$, and the dual of $X(w)$. However, Pech's calculations for $\mathrm{QH}^{*}(\mathrm{IG}(2,5))$ include

$$
[X(3,-1)] \star[X(3,-1)]=[X(3,1)]-q
$$

and

$$
[X(2,1)] \star[X(3,-1)]=-[X(3,2)]+q[X(1)]
$$

where negative coefficients appear. Thus, the coefficients are not enumerative. Moreover, since the coefficients are not enumerative we can conclude that $\operatorname{IG}(k, 2 n+1)$ is not a homogeneous space for $1<k<n+1$. The non-enumerativity of the coefficients is a consequence of not being able to use transversality. Pech is able to prove a Chevalley formula (she actually proved a Pieri rule) for $\operatorname{IG}(2,2 n+1)$ using a Kleiman-Bertini type lemma proved by Graber in [30] to show that some coefficients contain enumerative data. Difficulties arise when Pech's technique is attempted for cases $k>2$. In particular, Pech presents a conjecture in [55] that implies the structure constants in the quantum Chevalley formula that correspond curves of degree larger or equal to 2 vanish. We use the technique of curve neighborhoods to circumvent problems that nontransversality causes to prove that those structure constants vanish.

In joint work with Mihalcea we prove the following Chevalley formula.

Theorem 1.2.3. (quantum Chevalley formula [48]) For all $\lambda \in \Lambda$ we have

$$
[X(1)] \star[X(\lambda)]=\text { Classical Part }+q\left[X\left(\lambda^{*}\right)\right]+q\left[X\left(\lambda^{* *}\right)\right]
$$

The terms for $\lambda^{*}$ or $\lambda^{* *}$ are omitted if they do not exist..

This result recovers Pech's results in $[55,56]$ for $\operatorname{IG}(2,2 n+1)$ and we verify her conjecture for $\operatorname{IG}(3,2 n+1)$ in [55]. In addition, we recover the results for $\operatorname{IG}(n+$ $1,2 n+1) \cong \operatorname{IG}(n, 2 n)$ and $\mathrm{IG}(1,2 n+1) \cong \mathbb{P}^{2 n}$. The classical part is known by work done by Pech in [55] and Buch, Kresch, and Tamvakis in [15] using the theory of $(n-k)$-strict partitions (see Definition 11.2.1). Next, we define $\lambda^{*}$ and $\lambda^{* *}$.

If $\lambda_{1}=2 n+1-k$ and $\lambda_{k} \geq 0$ then let

$$
\lambda^{*}=\left(\lambda_{2} \geq \lambda_{3} \geq \cdots \geq \lambda_{k} \geq 0\right) .
$$

If $\lambda_{1}<2 n+1-k$ or $\lambda_{k}=-1$ then $\lambda^{*}$ does not exist. If $\lambda_{1}=2 n+1-k$ and $\lambda_{2}=2 n-k$ then let

$$
\lambda^{* *}=\left(\lambda_{1} \geq \lambda_{3} \geq \cdots \geq \lambda_{k} \geq-1\right) .
$$

If $\lambda_{2}<2 n-k$ then $\lambda^{* *}$ does not exist.

Example 1.2.4. Consider $n=4$ and $k=3$. For $\lambda=(6,5,1)$ our quantum components are indexed by $(6,1,-1)$ and $(5,1,0)$ which is exhibited in the first row of the following table. 


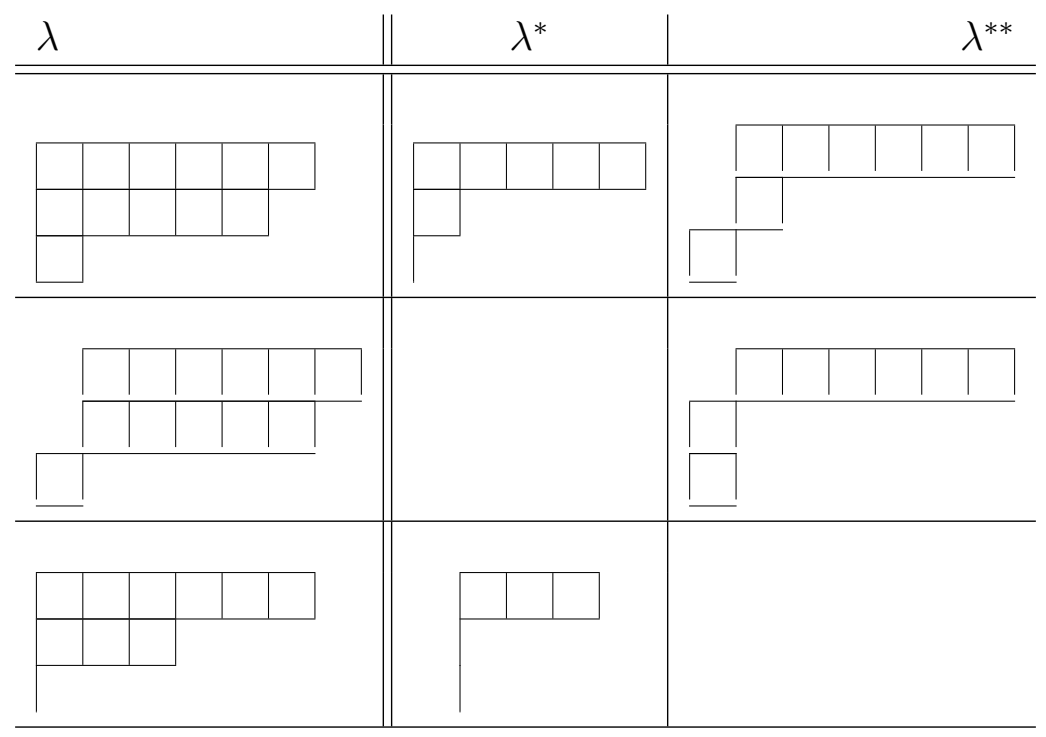

Even though the divisor class $[X(1)]$ does not generate the ring $\mathrm{QH}_{T}^{*}(\mathrm{IG}(k, 2 n+$ $1)$ ), the Chevalley rule is sufficient to produce a recursive formula to complete a multiplication table. The Chevalley formula has been used to calculate the equivariant quantum cohomology rings. Okounkov presented some of the ideas in [54] along with Molev and Sagan in [52]. These calculations have been used by Knutson and Tao in the equivariant case in [37]; Mihalcea in the equivariant quantum case in [50,51]; and by Buch, Chaput, Mihalcea, and Perrin in the equivariant quantum $K$-theory of cominuscule varieties in [8]. The recursive formula we obtain is

$$
\begin{aligned}
\left(c_{(1), \mu}^{\mu, 0}-c_{(1), \lambda}^{\lambda, 0}\right) c_{\lambda, \zeta}^{\mu, d} & =\sum_{\substack{\left(\lambda+1^{k}\right) \rightarrow\left(\delta+1^{k}\right) \\
|\delta|=|\lambda|+1}} 2^{N\left(\lambda+1^{k}, \delta+1^{k}\right)} c_{\delta, \zeta}^{\mu, d}-\sum_{\substack{\left(\gamma+1^{k}\right) \rightarrow\left(\mu+1^{k}\right) \\
|\mu|=|\gamma|+1}} 2^{N\left(\gamma+1^{k}, \mu+1^{k}\right)} c_{\lambda, \zeta}^{\gamma, d} \\
& +c_{\lambda^{*}, \zeta}^{\mu, d-1}+c_{\lambda^{* *}, \zeta}^{\mu, d-1}-c_{\lambda, \zeta}^{\mu^{+}, d-1}-c_{\lambda, \zeta}^{\mu^{++}, d-1}
\end{aligned}
$$

The key ingredient in our proof of the Chevalley formula is the technique of curve neighborhoods which circumvents problems that arise from non-transversality. We define briefly the notion of curve neighborhoods where $X(w) \subset \operatorname{IG}(k, 2 n+1)$ is a Schubert variety. Then the degree $d$ curve neighborhood of $X(w)$ is $\Gamma_{d}(X(w))=$ $\mathrm{ev}_{2}\left(\mathrm{ev}_{1}^{-1}(X(w))\right)$ where, recall, $\mathrm{ev}_{i}$ are evaluation maps. As a set, it is the closure of the locus of points of degree $d$ rational curves that intersect the Schubert variety $X(w)$. More about curve neighborhoods can be found in $[9,16]$. The combinatorial tool we use to calculate the curve neighborhoods is the weighted moment graph where the vertices are the torus fixed points and the edges are the torus stable curves whose label is the curve's degree. The weighted moment graph of $\operatorname{IG}(k, 2 n+1)$ is a subgraph of the weighted moment graph of $\operatorname{IG}(k, 2 n+2)$. 
Figure 1.1: The figure is the moment graph of $\operatorname{IG}(2,6)$ without degree labels. The blue vertices and edges are not included in the Schubert variety $\operatorname{IG}(2,5)$. Also, the red vertices and edges are in the closed orbit which is isomorphic to $\operatorname{IG}(1,4)$. The vertices are labeled by the elements of $W^{2 n+1}$ for $k=n=2$.

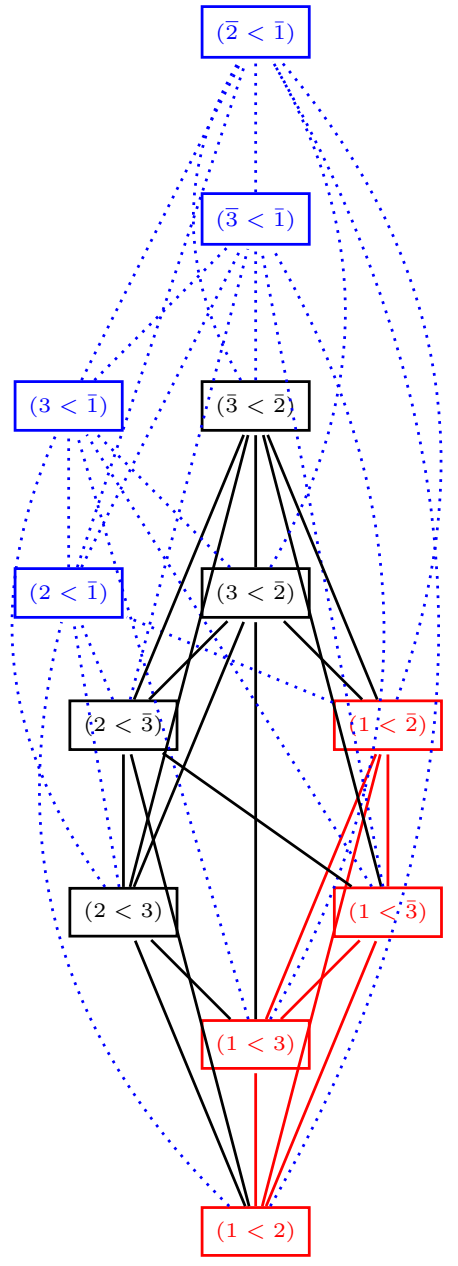

We make use of the following combinatorial statement which translates geometry into a combinatorial statement. Consider the non-oriented weighted moment graph of $\operatorname{IG}(k, 2 n+1)$. Let $\left\{v^{1}, \cdots, v^{s}\right\}$ be the maximal vertices with respect to the Bruhat order in the weighted moment graph which can be reached from any $u \leq w$ using a path of degree $d$ or less. Then $\Gamma_{d}(X(w))=X\left(v^{1}\right) \cup \cdots \cup X\left(v^{s}\right)$. 
Figure 1.2: The figure is the weighted moment graph of $\operatorname{IG}(2,5)$. The thick edges have degree 2 . Observe $\Gamma_{1}(X(1<2))=X(2<\overline{3}) \cup X(1<\overline{2})$.

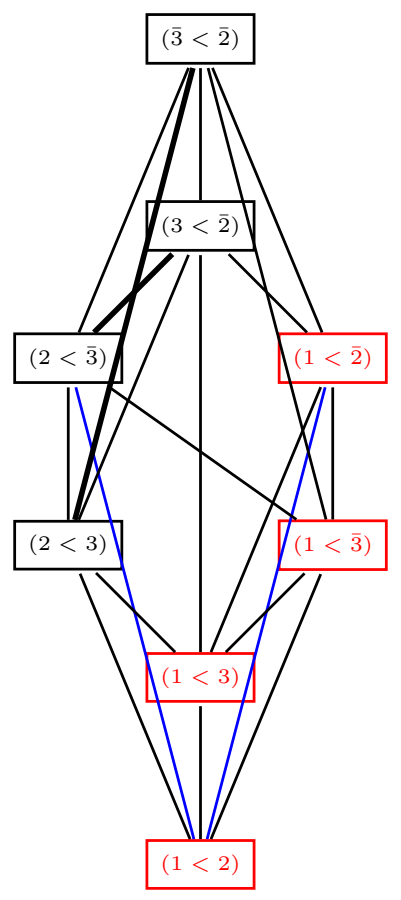

A curve neighborhood of any Schubert variety in a homogeneous space $G / P$ is irreducible. In contrast, observe $\Gamma_{1}(X(1<2))=X(2<\overline{3}) \cup X(1<\overline{2})$ in $\operatorname{IG}(2,5)$ is reducible by using Figure 1.2.1 and the combinatorial statement about curve neighborhoods. Reducible curve neighborhoods are a new phenomenon and a common occurrence in $\mathrm{IG}(k, 2 n+1)$.

We need explicit calculations of the line neighborhoods $\Gamma_{1}(X(w))$ where $X(w)$ is a Schubert variety in closed the orbit of $\operatorname{IG}(k, 2 n+1)$. The Hecke product is used for this calculation. For a simple reflection $s_{i}$ the Hecke product is

$$
w \cdot s_{i}=\left\{\begin{array}{lr}
w s_{i} & \text { if } \ell\left(w s_{i}\right)>\ell(w) \\
w & \text { otherwise }
\end{array} .\right.
$$

The product $w \cdot v$ is defined to be reduced if $\ell(w v)=\ell(w)+\ell(v)$. Consider the following special elements of $W^{2 n+1}$

$$
O_{1}=(2<3<\cdots<k<\overline{k+1})
$$

and

$$
O_{2}=(1<3<\cdots<k<\overline{2})
$$

We have the following theorem. 
Theorem 1.2.5. For any $w \in W^{2 n+1}$ where $X(w) \subset X_{c}$ we have that

$$
\Gamma_{1}(X(w))=X\left(w \cdot O_{1}\right) \cup X\left(w \cdot O_{2}\right) .
$$

We use that $\Gamma_{1}(p t)=X\left(O_{1}\right) \cup X\left(O_{2}\right)$ and $\ell\left(O_{1}\right)=\ell\left(O_{2}\right)=\operatorname{deg} q-1$ in the proof. The following Theorem guarantees vanishing in the Chevalley formula when certain conditions on the dimension of the curve neighborhoods are satisfied. Let $[X(w)]_{T}$ denote the equivariant fundamental class.

Theorem 1.2.6. Let $d \geq 1$ be a degree in $H_{2}(\operatorname{IG}(k, 2 n+1))$.

1. For all $w \in W^{2 n+1}$ we have $\operatorname{dim} \Gamma_{d}(X(w))-\operatorname{dim} X(w) \leq d \operatorname{deg} q-1$.

2. Let $X(v), X(w) \subset \mathrm{IG}(k, 2 n+1)$ be two Schubert varieties and $X($ Div $)$ the Schubert divisor. If $\operatorname{dim} \Gamma_{d}(X(v))<\ell(v)+\operatorname{deg} q^{d}-1$ then the equivariant Gromov-Witten invariant

$$
\left\langle[X(D i v)]_{T},[X(v)]_{T},[X(w)]_{T}^{\vee}\right\rangle_{d}=0
$$

where $[X(w)]_{T}^{\vee}$ is the Poincare dual of $[X(w)]_{T}$. In particular, the equivariant Gromov-Witten invariant above vanishes if either $d \geq 2$, or $d=1$ and $X(v)$ is not included in the closed orbit $X_{c} \subset \mathrm{IG}(k, 2 n+1)$.

The remaining invariants, $c_{D i v, v}^{w, 1}$ where $v \in X_{c}$, require a separate calculation. We make use of the next theorem

Theorem 1.2.7. Let $z \in\left\{O_{1}, O_{2}\right\}$ and $w \in W^{2 n+1}$ such that $w \in X_{c}$. Then

$$
\operatorname{dim} X(w \cdot z)-\operatorname{dim} X(w)=\operatorname{deg} q-1
$$

if and only if the product $w \cdot z$ is reduced and $w \cdot z$ is a minimal length representative in $W^{2 n+1}$.

In order to prove the coefficients of the quantum terms in the Chevalley formula are one we need to analyze some geometric properties of the moduli spaces of stable maps $\overline{\mathcal{M}}_{0, r}(\mathrm{IG}(k, 2 n+1), 1) \rightarrow \operatorname{IG}(k, 2 n+1)$ where $r=1,2$ and the Gromov-Witten varieties

$$
G W_{1}(w):=\operatorname{ev}_{1}^{-1}(X(w)) \subset \overline{\mathcal{M}}_{0,2}(\mathrm{IG}, 1) .
$$

Theorem 1.2.8. Consider the Schubert variety $X(w) \subset X_{c}$ (closed orbit). Then the Gromov-Witten variety $G W_{1}(w)$ has two irreducible components

$$
G W_{1}(w)=G W_{1}^{(1)}(w) \cup G W_{1}^{(2)}(w),
$$

where $G W_{1}^{(1)}(w)$ is the closure of the subvariety corresponding to lines $L$ such that $L \cap X^{\circ} \neq \emptyset$, and $G W_{1}^{(2)}(w)$ is the closed subscheme corresponding to lines $L$ included in the closed orbit $X_{c}$. Further, each irreducible component is generically smooth and it has dimension $\operatorname{dim} \overline{\mathcal{M}}_{0,2}(\mathrm{IG}(k, 2 n+1), 1)-\operatorname{codim}_{\mathrm{IG}(k, 2 n+1)} X(w)$. 
We prove that if $w \cdot O_{1}$ is reduced and a minimal length representative then

$$
\mathrm{ev}_{2}: G W_{1}^{(1)}(w) \rightarrow X\left(w \cdot O_{1}\right)
$$

is a birational morphism. This implies, by definition of Gromov-Witten invariants, that $c_{D i v, w}^{w \cdot O_{1}, 1}=1$. It follows from a result in [9] that if $w \cdot O_{2}$ is reduced and a minimal length representative then

$$
\mathrm{ev}_{2}: G W_{1}^{(2)}(w) \rightarrow X\left(w \cdot O_{2}\right)
$$

is a birational morphism. Therefore, $c_{D i v, w}^{w \cdot O_{2}, 1}=1$. 


\section{Chapter 2}

\section{Preliminaries}

\subsection{The odd symplectic group}

We recall next the definition and basic properties of odd symplectic flag manifolds, following Mihai's paper [46]; see also [47,56]. Let $E$ be a complex vector space of dimension $\operatorname{dim}_{\mathbb{C}} E=2 n+1$, and let $\omega$ be a general symplectic form on $E$, i.e. a bilinear and skew-symmetric. Such a form is necessarily degenerate, with kernel of dimenson 1. The odd symplectic group is the subgroup of $\mathrm{GL}(E)$ which preserves this symplectic form:

$$
\operatorname{Sp}_{2 n+1}(E):=\{g \in \mathrm{GL}(E): \omega(g \cdot u, g \cdot v)=\omega(u, v), \forall u, v \in E\} .
$$

It will be convenient to extend the form $\omega$ to a non-degenerate symplectic form $\widetilde{\omega}$ on an even dimensional space $\widetilde{E} \supset E$, and to identify $E \subset \widetilde{E}$ with a coordinate hyperplane $\mathbb{C}^{2 n+1} \subset \mathbb{C}^{2 n+2}$. For that, let $\left\{\mathbf{e}_{1}, \mathbf{e}_{2}, \cdots, \mathbf{e}_{2 n+2}\right\}$ be the standard basis of $\widetilde{E}:=\mathbb{C}^{2 n+2}$. Let $\bar{i}=2 n+3-i$ and $|i|=\min \{i, \bar{i}\}$ and consider $\widetilde{\omega}$ to be the non-degenerate symplectic form on $\widetilde{E}$ defined by

$$
\widetilde{\omega}\left(\mathbf{e}_{i}, \mathbf{e}_{j}\right)=\delta_{i, \bar{j}} \text { for all } 1 \leq i \leq j \leq \overline{1} .
$$

The form $\widetilde{\omega}$ restricts to the degenerate symplectic form $\omega$ on $E:=\mathbb{C}^{2 n+1}=\left\langle\mathbf{e}_{1}, \mathbf{e}_{2}, \cdots, \mathbf{e}_{2 n+1}\right\rangle$ such that the kernel ker $\omega$ is generated by $\mathbf{e}_{1}$. Then

$$
\omega\left(\mathbf{e}_{i}, \mathbf{e}_{j}\right)=\delta_{i, \bar{j}} \text { for all } 1 \leq i \leq j \leq \overline{2} .
$$

Let $F \subset E$ denote the $2 n$ dimensional vector space with basis $\left\{\mathbf{e}_{2}, \mathbf{e}_{3}, \cdots, \mathbf{e}_{2 n+1}\right\}$. Since $F \cap \operatorname{ker} \omega=(0)$ it follows that $\omega$ restricts to a non-degenerate form on $F$. Let $\mathrm{Sp}_{2 n}(F)$ and $\mathrm{Sp}_{2 n+2}(\widetilde{E})$ denote the symplectic groups which preserve respectively the symplectic form $\omega_{\mid F}$ and $\widetilde{\omega}$. Then with respect to the decomposition $E=F \oplus \operatorname{ker} \omega$ the elements of the odd-symplectic group $\operatorname{Sp}_{2 n+1}(E)$ are matrices of the form

$$
\operatorname{Sp}_{2 n+1}(E)=\left\{\left(\begin{array}{cc}
\lambda & a \\
0 & S
\end{array}\right): \lambda \in \mathbb{C}^{*}, a \in \mathbb{C}^{2 n}, S \in \operatorname{Sp}_{2 n}(F)\right\} .
$$


The symplectic group $\operatorname{Sp}_{2 n}(F)$ embeds naturally into $\operatorname{Sp}_{2 n+1}(E)$ by $\lambda=1$ and $a=0$, but $\operatorname{Sp}_{2 n+1}(E)$ is not a subgroup of $\operatorname{Sp}_{2 n+2}(\widetilde{E}) .{ }^{1}$ Mihai showed in [46, Prop. 3.3] that there is a surjection $P \rightarrow \operatorname{Sp}_{2 n+1}(E)$ where $P \subset \operatorname{Sp}_{2 n+2}(\widetilde{E})$ is the parabolic subgroup which preserves ker $\omega$, and the map is given by restricting $g \mapsto g_{\mid E}$. Then the Borel subgroup of $B_{2 n+2} \subset \operatorname{Sp}_{2 n+2}(\widetilde{E})$ of upper triangular matrices restricts to the (Borel) subgroup $B \subset \operatorname{Sp}_{2 n+1}(E)$. Similarly, the maximal torus $T_{2 n+2}:=$ $\left\{\operatorname{diag}\left(t_{1}, \cdots, t_{n+1}, t_{n+1}^{-1}, \cdots, t_{1}^{-1}\right): t_{1}, \cdots, t_{n+1} \in \mathbb{C}^{*}\right\} \subset B_{2 n+2}$ restricts to the maximal torus

$$
T=\left\{\operatorname{diag}\left(t_{1}, \cdots, t_{n+1}, t_{n+1}^{-1}, \cdots, t_{2}^{-1}\right): t_{1}, \cdots, t_{n+1} \in \mathbb{C}^{*}\right\} \subset B
$$

\subsection{The odd symplectic flag varieties}

Let $1 \leq i_{1}<\ldots<i_{r} \leq n+1$. The odd symplectic flag variety $\operatorname{IF}\left(i_{1}, \ldots, i_{r} ; E\right)$ consists of flags of linear subspaces $F_{i_{1}} \subset \ldots \subset F_{i_{k}} \subset E$ such that $\operatorname{dim} F_{i_{j}}=i_{j}$ and $F_{i_{j}}$ is isotropic with respect to the symplectic form $\omega$. The inclusion $E \subset \widetilde{E}$ makes it a closed subvariety of the (even) symplectic flag variety $\operatorname{IF}\left(i_{1}, \ldots, i_{r} ; \widetilde{E}\right)$ which consists of similar flags of subspaces, isotropic with respect to the symplectic form $\widetilde{\omega}$. The latter is a homogeneous space for $\operatorname{Sp}_{2 n+2}(\widetilde{E})$. In fact, the inclusions $F \subset E \subset \widetilde{E}$ realize the odd-symplectic flag variety as an "intermediate" variety between two consecutive symplectic flag varieties:

$$
\operatorname{IF}\left(i_{1}-1, \ldots, i_{r}-1 ; F\right) \subset \operatorname{IF}\left(i_{1}, \ldots, i_{r} ; E\right) \subset \operatorname{IF}\left(i_{1}, \ldots, i_{r} ; \widetilde{E}\right),
$$

where the flags in $\operatorname{IF}\left(i_{1}-1, \ldots, i_{r}-1 ; F\right)$ are isotropic with respect to $\omega_{\mid F}$. In this paper we will focus mostly on the Grassmannian case, i.e. when $r=1$. The corresponding symplectic Grassmannians are

$$
\mathrm{IG}(k-1, F) \subset \mathrm{IG}(k, E) \subset \operatorname{IG}(k, \widetilde{E}) .
$$

There is a natural embedding of the odd symplectic flag variety as a closed subvariety of the type A partial flag variety $\mathrm{Fl}\left(i_{1}, \ldots, i_{r} ; E\right)$ which parametrizes flags of given dimensions in $E$. In this situation $\operatorname{IF}\left(i_{1}, \ldots, i_{r} ; E\right)$ is the zero locus of a certain global section of a vector bundle on $\operatorname{Fl}\left(i_{1}, \ldots, i_{r} ; E\right)$ induced by the symplectic form. Mihai used this to prove that that $\operatorname{IF}\left(i_{1}, \ldots, i_{r} ; E\right)$ is a smooth subvariety of $\operatorname{Fl}\left(i_{1}, \ldots, i_{r} ; E\right)$ of codimension $\frac{i_{r}\left(i_{r}-1\right)}{2}$; see [46, Prop. 4.1] for details.

The odd-symplectic group acts on $\operatorname{IF}\left(i_{1}, \ldots, i_{r} ; E\right)$, but the action is no longer transitive. In Proposiitons 4.5 and 4.6 from [46], Mihai described the orbits of this action in the case of the full flag variety, i.e. when $r=n+1$, and noted that the results extend in the obvious way to partial flag varieties. We recall this description next.

\footnotetext{
${ }^{1}$ However, Gelfand and Zelevinsky [26] defined another group ${\widetilde{\mathrm{Sp}_{2 n+1}}}_{2 l o s e l y}$ related to $\mathrm{Sp}_{2 n+1}$ such that $\mathrm{Sp}_{2 n} \subset \widetilde{\mathrm{Sp}}_{2 n+1} \subset \mathrm{Sp}_{2 n+2}$.
} 
Proposition 2.2.1. The odd symplectic group $\operatorname{Sp}_{2 n+1}(E)$ acts on $\operatorname{IF}\left(i_{1}, \cdots, i_{r} ; E\right)$ with $r+1$ orbits if $i_{r}<n+1$ and $r$ orbits if $i_{r}=n+1$. The orbits are:

$$
\begin{aligned}
\mathcal{O}_{j} & =\left\{V_{i_{1}} \subset \cdots \subset V_{i_{r}} \subset E: e_{1} \in V_{i_{j}}, e_{1} \notin V_{i_{j-1}}\right\} \text { for all } 1 \leq j \leq r \\
\text { and } \mathcal{O}_{r+1} & =\left\{V_{i_{1}} \subset \cdots \subset V_{i_{r}} \subset E: e_{1} \notin V_{i_{r}}\right\} \text { if } i_{r}<n+1,
\end{aligned}
$$

where by convention $V_{i_{0}}=(0)$. The only closed orbit is $\mathcal{O}_{1}$, and it may be naturally identified to $\operatorname{IG}\left(i_{1}-1, \ldots, i_{r}-1 ; F\right)$.

In particular, for $1 \leq k \leq n$ the odd symplectic group $\operatorname{Sp}_{2 n+1}(E)$ acts on the odd symplectic Grassmannian $\mathrm{IG}(k, E)$ with two orbits

$$
\begin{aligned}
& X_{c}=\left\{V \in \operatorname{IG}(k, E): e_{1} \in V\right\} \text { the closed orbit } \\
& X^{\circ}=\left\{V \in \operatorname{IG}(k, E): e_{1} \notin V\right\} \text { the open orbit. }
\end{aligned}
$$

The closed orbit $X_{c}$ is isomorphic to $\mathrm{IG}(k-1, F)$. If $k=n+1$ then $\operatorname{IG}(n+1, E)=X_{c}$ may be identified to the Lagrangian Grassmannian $\mathrm{IG}(n, F)$.

Mihai identifies the closures $\overline{\mathcal{O}}_{i}$ of the orbits and proves they are smooth.

Proof. By [46, Proposition 4.5] the odd symplectic group $\mathrm{Sp}_{2 n+1}$ acts on the full odd symplectic flag $\operatorname{IF}(2 n+1)$ with $n+1$ orbits

$$
X_{i}=\left\{\left(V_{1} \subset \cdots \subset V_{n+1}\right) \in \operatorname{IF}(2 n+1): e_{1} \in V_{i}, e_{1} \notin V_{i-1}\right\}, 1 \leq i \leq n+1 .
$$

Each $K_{j}$ is clearly $\mathrm{Sp}_{2 n+1}$-stable. Let $V_{\bullet}^{1}, V_{\bullet}^{2} \in K_{j}$. Then there exists a $g \in$ $\mathrm{Sp}_{2 n+1}$ where $g V_{i_{j}}^{1}=V_{i_{j}}^{2}$. Thus $\mathrm{Sp}_{2 n+1}$ acts transitively on $K_{j}$. It is clear that $\operatorname{IF}\left(i_{1}, \cdots, i_{r} ; 2 n+1\right)=\bigcup K_{j}$. The result [46, Proposition 4.3] proves $X_{c}$ is isomorphic to $\operatorname{IG}(k-1,2 n)$. The orbits are distinct. Indeed, let $X_{i}$ and $X_{j}$ be two obits where $i<j$ and $V=\left(V_{1} \subset \cdots \subset V_{n+1}\right) \in X_{i} \cap X_{j}$. This implies that $e_{1} \in V_{i}$ since $V \in X_{i}$ but $e_{i} \notin V_{i}$ since $V \in X_{j}$. The result follows.

From now on we will identify $F \subset E \subset \widetilde{E}$ to $\mathbb{C}^{2 n} \subset \mathbb{C}^{2 n+1} \subset \mathbb{C}^{2 n+2}$ with bases $\left\langle\mathbf{e}_{2}, \ldots, \mathbf{e}_{2 n+1}\right\rangle \subset\left\langle\mathbf{e}_{1}, \ldots, \mathbf{e}_{2 n+1}\right\rangle \subset\left\langle\mathbf{e}_{1}, \ldots, \mathbf{e}_{2 n+2}\right\rangle$. The corresponding symplectic flag manifolds will be denoted by $\operatorname{IF}\left(i_{1}-1, \ldots, i_{r}-1 ; 2 n\right) \subset \operatorname{IF}\left(i_{1}, \ldots, i_{r} ; 2 n+1\right) \subset$ $\operatorname{IF}\left(i_{1}, \ldots, i_{r} ; 2 n+2\right)$. Similarly $\operatorname{Sp}_{2 n+1}(E)$ will be denoted by $\operatorname{Sp}_{2 n+1}$ etc.

\subsection{The Weyl group and odd-symplectic minimal representatives}

We recall next the sets which we will use in the next section to define the Schubert varieties (cf. [25,32,33]).

Consider the root system of type $C_{n+1}$ with positive roots $R^{+}=\left\{t_{i} \pm t_{j}: 1 \leq\right.$ $i<j \leq n+1\} \cup\left\{2 t_{i}: 1 \leq i \leq n+1\right\}$ and the subset of simple roots $\Delta=\left\{\alpha_{i}:=\right.$ $\left.t_{i}-t_{i+1}: 1 \leq i \leq n\right\} \cup\left\{\alpha_{n+1}:=2 t_{n+1}\right\}$. The associated Weyl group $W$ is the 
hyperoctahedral group consisting of signed permutations, i.e. permutations $w$ of the elements $\{1, \cdots, n+1, \overline{n+1}, \cdots, \overline{1}\}$ satisfying $w(\bar{i})=\overline{w(i)}$ for all $w \in W$. For $1 \leq i \leq n$ denote by $s_{i}$ the simple reflection corresponding to the root $t_{i}-t_{i+1}$ and $s_{n+1}$ the simple reflection of $2 t_{n+1}$. Each subset $I:=\left\{i_{1}<\ldots<i_{r}\right\} \subset\{1, \ldots, n+1\}$ determines a parabolic subgroup $P:=P_{I} \leq \operatorname{Sp}_{2 n+2}(\widetilde{E})$ with Weyl group $W_{P}=$ $\left\langle s_{i}: i \neq i_{j}\right\rangle$ generated by reflections with indices not in $I$. Let $\Delta_{P}:=\left\{\alpha_{i_{s}}: i_{s} \notin\right.$ $\left.\left\{i_{1}, \ldots, i_{r}\right\}\right\}$ and $R_{P}^{+}:=\operatorname{Span}_{\mathbb{Z}} \Delta_{P} \cap R^{+}$; these are the positive roots of $P$. Let $\ell: W \rightarrow \mathbb{N}$ be the length function and denote by $W^{P}$ the set of minimal length representatives of the cosets in $W / W_{P}$. We have a natural ordering

$$
1<2<\ldots<n+1<\overline{n+1}<\ldots<\overline{1}
$$

which is consistent with our earlier notation $\bar{k}:=2 n+3-k$. It is easy to see that the minimal length representatives are as follows.

Lemma 2.3.1. The set of minimal length representatives $W^{P}$ of the cosets of $W / W_{P}$ consists of those $w \in W$ such that if $i_{r}<n+1$ then

$$
\begin{aligned}
w(1)< & \cdots<w\left(i_{1}\right), \\
w\left(i_{1}+1\right)< & \cdots<w\left(i_{2}\right), \\
& \vdots \\
w\left(i_{r}+1\right)< & \cdots<w(n+1) \leq n+1 .
\end{aligned}
$$

If $i_{r}=n+1$ then

$$
\begin{aligned}
& w(1)<\cdots<w\left(i_{1}\right), \\
& w\left(i_{1}+1\right)<\cdots<w\left(i_{2}\right), \\
& w\left(i_{r-1}+1\right)<\cdots<w\left(i_{r}\right)=w(n+1) .
\end{aligned}
$$

Since $w$ is a signed permutation, the conditions above determine the last row uniquely, and the minimal length representatives will be denoted by

$$
\left(w(1)<\cdots<w\left(i_{1}\right)\left|w\left(i_{1}+1\right)<\cdots<w\left(i_{2}\right)\right| \cdots \mid w\left(i_{r-1}+1\right)<\cdots<w\left(i_{r}\right)\right) .
$$

In particular, if $P=P_{k}$ is the maximal parabolic obtained by excluding the reflection $s_{k}$, then the minimal length representatives $W^{P_{k}}$ have the form $(w(1)<w(2)<$ $\cdots<w(k))$. The Weyl group $W$ admits a partial ordering $\leq$ given by the Bruhat order. Its covering relations are given by $w<w s_{\alpha}$ where $\alpha \in R^{+}$is a root and $\ell(w)<\ell\left(w s_{\alpha}\right)$. Let $w_{0} \in W$ be the signed permutation $w_{0}=(\overline{2}, \overline{3}, \ldots, \overline{n+1}, 1)$. We will see in the next section that $w_{0}$ is the index of the Schubert variety corresponding to the odd-symplectic (partial) flag manifold inside the even symplectic flag manifold. In order to index the Schubert varieties in the odd-symplectic flag manifold, we consider the set $W^{2 n+1}:=\left\{w \in W: w \leq w_{0}\right\}$. In terms of signed permutations, $W^{2 n+1}$ 
contains those elements $w=(w(1)<\cdots<w(k))$ such that $1 \leq w(i) \leq \overline{2}$ for all $1 \leq i \leq k$. In the maximal parabolic case, the minimal length representative for the coset of $w_{0}$ in $W^{P_{k}}$ corresponds to the $(n+1-k)$-strict partition $1^{k}:=(1,1, \ldots, 1)$ if $k<n+1$ and to $(k, 0, \ldots, 0)$ if $k=n+1$.

Example 2.3.2. The reflection $s_{t_{1}+t_{2}}$ is the signed permutation $s_{t_{1}+t_{2}}(1)=\overline{2}, s_{t_{1}+t_{2}}(2)=$ $\overline{1}$, and $s_{t_{1}+t_{2}}(i)=i$ for all $3 \leq i \leq n+1$. The minimal length representative of $s_{t_{1}+t_{2}} W_{P_{k}}$ is $(3<4<\cdots<k<\overline{2}<\overline{1})$.

\subsubsection{Type A and Type C Bruhat order compatibility}

In this section we prove that the Bruhat order on elements in $W$ for the Type $\mathrm{C}$ case is compatible with the Bruhat order on $S_{2 n+2}$ for Type A case. For this subsection only, let $\leq_{C}$ denote the Bruhat order on $W$ and $\leq_{A}$ denote the Bruhat order on $S_{2 n+2}$. The main result is:

Proposition 2.3.3. Let $v, w \in W \subset S_{2 n+2}$. Then $v \leq_{C} w$ if and only if $v \leq_{A} w$. In particular, we can use the following characterization known for type A: for any $v, w \in W^{P}$,

$$
v=\left(v_{1}<v_{2}<\cdots<v_{k}\right) \leq w=\left(w_{1}<w_{2}<\cdots<w_{k}\right)
$$

if and only if $v_{i} \leq w_{i}$ for all $1 \leq i \leq k$.

Before we prove that proposition we need set up some notation and prove a few Lemmas. Let $R_{A}^{+}=\left\{e_{i} \pm e_{j}: 1 \leq i<j \leq 2 n+2\right\}$ be the positive roots for Type A. Let $s_{1}^{A}, s_{2}^{A}, \cdots, s_{2 n+1}^{A}$ represent the simple reflections for $S_{2 n+2}$ where $s_{i}^{A}=s_{e_{i}-e_{i+1}}^{A}=$ $(i, i+1)$ for $1 \leq i \leq 2 n+1$. Recall the length of $w \in S_{2 n+2}$ is $\ell_{A}(w)=\#\left\{\alpha \in R_{A}^{+}\right.$: $w(\alpha)<0\}$ and the length of $w \in W$ is $\ell(w)=\#\left\{\alpha \in R^{+}: w(\alpha)<0\right\}$.

For all $w \in W$ we have the following equivalences

$$
\begin{aligned}
w\left(t_{i}-t_{j}\right)<0 & \Leftrightarrow w\left(e_{i}-e_{j}\right)<0 \text { and } w\left(e_{\bar{j}}+e_{\bar{i}}\right)<0 \text { for } 1 \leq i<j \leq n+1, \\
w\left(t_{i}+t_{j}\right)<0 & \Leftrightarrow w\left(e_{i}-e_{\bar{j}}\right)<0 \text { and } w\left(e_{j}-e_{\bar{i}}\right)<0 \text { for } 1 \leq i<j \leq n+1, \\
w\left(2 t_{i}\right)<0 & \Leftrightarrow w\left(e_{i}-e_{\bar{i}}\right)<0 \text { for } 1 \leq i \leq n+1 .
\end{aligned}
$$

We also have the following equalities

$$
\begin{aligned}
s_{1} & =s_{1}^{A} s_{\overline{1}-1}^{A}, \\
& \vdots \\
s_{n} & =s_{n}^{A} s_{\bar{n}-1}^{A}, \\
s_{n+1} & =s_{n+1}^{A} .
\end{aligned}
$$

Given a reduced word $s_{i_{1}} \ldots s_{i_{t}} \in W$, we can obtain a word in $S_{2 n+2}$ with the $s_{i}^{A}$ 's using the equalities above. We need to show that this new word is also reduced. We will use from Humphreys' book [33, Proposition on page 10] that states $s_{i}\left(R^{+}-\left\{t_{i}-t_{i+1}\right\}\right)=$ 
$R^{+}-\left\{t_{i}-t_{i+1}\right\}, s_{n+1}\left(R^{+}-\left\{2 t_{n+1}\right\}\right)=R^{+}-\left\{2 t_{n+1}\right\}$, and $s_{i}^{A}\left(R_{A}^{+}-\left\{e_{i}-e_{i+1}\right\}\right)=$ $R_{A}^{+}-\left\{e_{i}-e_{i+1}\right\}$. Also, we use the type independent result from [33] that states that $v s_{i}>v$ if and only if $v\left(\alpha_{i}\right)>0$ where $\alpha_{i}$ is the $i$ th simple root.

Lemma 2.3.4. Let $v \in W$.

1. If $1 \leq i \leq n$ and $\ell\left(v s_{i}\right)=\ell(v)+1$ then $\ell_{A}\left(v s_{i}^{A} s_{\overline{1}-1}^{A}\right)=\ell_{A}(v)+2$.

2. If $\ell\left(v s_{n+1}\right)=\ell(v)+1$ then $\ell_{A}\left(v s_{n+1}^{A}\right)=\ell_{A}(v)+1$.

Proof. For case (1) let $1 \leq i \leq n$ and $\ell\left(v s_{i}\right)=\ell(v)+1$. We must have $v\left(t_{i}-t_{i+1}\right)>0$. Then $v\left(t_{i}-t_{i+1}\right)>0$ implies $v\left(e_{i}-e_{i+1}\right)>0$. That is, we have $\ell_{A}\left(v s_{i}^{A}\right)=\ell_{A}(v)+1$. Thus, $v\left(t_{i}-t_{i+1}\right)>0$ implies $\left(v s_{i}^{A}\right)\left(e_{\bar{i}-1}-e_{\bar{i}}\right)>0$. That is, we have $\ell_{A}\left(v s_{i}^{A} s_{\bar{i}-1}^{A}\right)=$ $\ell_{A}(v)+2$.

For case (2) let $\ell\left(v s_{n+1}\right)=\ell(v)+1$. We must have $v\left(2 t_{n+1}\right)>0$. Then $v\left(2 t_{n+1}\right)>0$ implies $v\left(e_{n+1}-e_{n+2}\right)>0$. That is, we have $\ell_{A}\left(v s_{i}^{A}\right)=\ell_{A}(v)+1$. The result follows.

Lemma 2.3.5. Let $s_{i_{1}} \ldots s_{i_{t}} \in W$ be a reduced word. The word that is produced by replacing $s_{i_{j}}$ with $s_{i_{j}}^{A} s_{i_{j}-1}^{A}$, if $1 \leq i_{j} \leq n$, and replacing $s_{n+1}$ with $s_{n+1}^{A}$ is reduced.

Proof. The proof is by induction. For the base case it is clear that $\ell\left(s_{1} s_{1}\right)=$ $\ell_{A}\left(s_{1}^{A} s_{1}^{A}\right)=0$. Suppose $s_{i_{1}} \ldots s_{i_{j_{1}}}=s_{i_{1}}^{A} \ldots s_{i_{j_{2}}}^{A}$ and $\ell\left(s_{i_{1}}^{A} \ldots s_{i_{j_{2}}}^{A}\right)=j_{2}$. Then we have the two cases as follows:

1. If $1 \leq i_{j_{1}+1} \leq n$ then $\ell\left(s_{i_{1}}^{A} \ldots s_{i_{j_{2}}}^{A} s_{i_{j_{1}+1}}^{A} s \frac{A}{i_{j_{1}+1}-1}\right)=j_{2}+2$,

2. if $i_{j_{1}+1}=n+1$ then $\ell\left(s_{i_{1}}^{A} \ldots s_{i_{j_{2}}}^{A} s_{i_{j_{1}+1}}^{A}\right)=j_{2}+1$.

The result follows.

Proof of Proposition 2.3.3. Given a reduced word in $W$ and a sub-word, we can produce corresponding reduced words in $S_{2 n+2}$ such that one word is a sub-word of the other.

\subsubsection{Hecke Product}

We will use the Hecke product on the Weyl group $W$. For a simple reflection $s_{i}$ the product is defined by

$$
w \cdot s_{i}=\left\{\begin{array}{lr}
w s_{i} & \text { if } \ell\left(w s_{i}\right)>\ell(w) \\
w & \text { otherwise }
\end{array}\right.
$$

The Hecke product gives $W$ a structure of an associative monoid. Given $u, v \in W$, the product $u v$ is called reduced if $\ell(u v)=\ell(u)+\ell(v)$. For any parabolic group $P:=P_{I}$, the Hecke product determines a left action $W \times W / W_{P} \longrightarrow W / W_{P}$ defined by

$$
u \cdot\left(w W_{P}\right)=(u \cdot w) W_{P} .
$$

We recall the following properties of the Hecke product (cf. e.g. [16]). 
Lemma 2.3.6. For any $u, v \in W$ there is an inequality $\ell\left(u \cdot v W_{P}\right) \leq \ell(u)+\ell\left(v W_{P}\right)$. If the equality holds then $u \cdot v W_{P}=u v W_{P}$. If furthermore $v \in W^{P}$ is a minimal length representative, then the following are equivalent:

(i) $\ell\left(u \cdot v W_{P}\right)=\ell(u)+\ell\left(v W_{P}\right)$;

(ii) $u \cdot v=u v, \ell(u \cdot w)=\ell(u)+\ell(v)$, and uv is a minimal length representative in $W^{P}$.

Proof. The first part of this lemma is explicitly stated in $[16, \S 3]$. For the equivalence, observe first that (ii) implies (i). For the converse, since $v \in W^{P}$,

$$
\ell(u \cdot v) \geq \ell\left(u \cdot v W_{P}\right)=\ell(u)+\ell\left(v W_{P}\right)=\ell(u)+\ell(v) \geq \ell(u \cdot v) .
$$

Thus $u \cdot v \in W^{P}$ and $\ell(u \cdot v)=\ell(u)+\ell(v)$. The last equality is equivalent to $u \cdot v=u v$ and this finishes the proof.

When the parabolic group will be clear from the context, we will often write $u \cdot v$ to mean $u \cdot v W_{P}$.

\subsection{Schubert Varieties in even and odd flag mani- folds}

Let $I:=\left\{i_{1}<\ldots<i_{r}\right\} \subset\{1, \ldots, n+1\}$ and the associated parabolic subgroup $P:=P_{I}$. The even symplectic flag manifold $\operatorname{IF}\left(i_{1}, \ldots, i_{r} ; 2 n+2\right)$ is a homogeneous space $\mathrm{Sp}_{2 n+2} / P$. For each $w \in W^{P}$ let $Y(w)^{\circ}:=B_{2 n+2} w B_{2 n+2} / P$ be the Schubert cell. This is isomorphic to the space $\mathbb{C}^{\ell(w)}$. Its closure $Y(w):=\overline{Y(w)^{\circ}}$ is the Schubert variety. Recall that the Bruhat ordering can be equivalently desctibed by $v \leq w$ if and only if $Y(v) \subset Y(w)$. The following results were proved by Mihai [46, §4]. Let $B:=B_{2 n+2} \cap \mathrm{Sp}_{2 n+1}$ be the odd-symplectic Borel subgroup.

Proposition 2.4.1. (a) The natural embedding

$$
\iota: \operatorname{IF}\left(i_{1}, \cdots, i_{r} ; 2 n+1\right) \hookrightarrow \operatorname{IF}\left(i_{1}, \cdots, i_{r} ; 2 n+2\right)
$$

identifies $\operatorname{IF}\left(i_{1}, \cdots, i_{r} ; 2 n+1\right)$ with the (smooth) Schubert subvariety

$$
Y\left(w_{0} W_{P}\right) \subset \operatorname{IF}\left(i_{1}, \cdots, i_{r} ; 2 n+2\right) .
$$

(b) The Schubert cells (i.e. the $B_{2 n+2}$-orbits) in $Y\left(w_{0}\right)$ coincide with the B-orbits in $\operatorname{IF}\left(i_{1}, \ldots, i_{r} ; 2 n+1\right)$. In particular, the B-orbits in $\operatorname{IF}\left(i_{1}, \ldots, i_{r} ; 2 n+1\right)$ are given by the Schubert cells $Y(w)^{\circ} \subset \operatorname{IF}\left(i_{1}, \ldots, i_{r} ; 2 n+2\right)$ such that $w \leq w_{0}$.

Recall that $W^{2 n+1}$ denotes the subset of $W$ consisting of those permutations $w \leq$ $w_{0}$. To emphasize that we discuss Schubert cells or varieties in the odd-symplectic case, for each $w \in W^{2 n+1} \cap W^{P}$ we denote by $X(w)^{\circ}$, and $X(w)$, the Schubert cell $Y(w)^{\circ}$ respectively the Schubert variety $Y(w)$. 
We will call a signed permutation $w \in W$ odd symplectic if $w \leq w_{0}$. Equivalently, $w(j) \neq \overline{1}$ for any $1 \leq j \leq n+1$. (The equivalence can be seen e.g. by looking at what are the $T$-fixed points included in the odd-symplectic flag variety.) The odd symplectic permutations index the Schubert varieties in the odd-symplectic flag manifold. The following closure property of the Hecke product on odd-symplectic permutations will be important later on.

Lemma 2.4.2. Let $u, v \in W$ be two odd-symplectic permutations, and assume that $u(1)=1$. Then $u v$ and $u \cdot v$ are odd-symplectic permutations.

Proof. We need to show that $(u v)(j) \neq \overline{1}$ and $(u \cdot v)(j) \neq \overline{1}$ for any $1 \leq j \leq n+1$. In the first situation, since $u(1)=1$, if $(u v)(j)=\overline{1}$ for some $1 \leq j \leq n+1$, then $v(j)=\overline{1}$, which contradicts that $v$ is odd-symplectic. For the second, consider the signed permutation $u^{\prime}:=(u \cdot v) v^{-1} \in W$. By [16, Prop. 3.1] we have that $u^{\prime} \leq u$ and $u^{\prime} v=u \cdot v$. The condition that $u^{\prime} \leq u$ implies that there is an inclusion of Schubert varieties $X\left(u^{\prime}\right) \subset X(u)$ in the full odd-symplectic flag manifold IF := $\operatorname{IF}(1,2, \ldots, n+1 ; 2 n+1)$. Further, the hypothesis that $u(1)=1$ implies that $X(u)$ is in the closed orbit of IF, thus $X\left(u^{\prime}\right)$ is in the closed orbit as well. This implies that $u^{\prime}(1)=1$. Then $u \cdot v=u^{\prime} v$ and since $u^{\prime}(1)=1$ the element $u^{\prime} v$ is again odd-symplectic, as claimed.

Let $k<n+1$. The odd-symplectic Grassmannian $\operatorname{IG}(k, 2 n+1)$ is the Schubert variety $Y\left(w_{0} W_{P_{k}}\right)$ in $\operatorname{IG}(k, 2 n+2)$, and it has complex codimension $k$. A Schubert variety $X(w)$ is included in the closed orbit $X_{c}$ of if and only if $w(1)=1$. 


\section{Chapter 3}

\section{(Equivariant) cohomology}

As before we fix a parabolic subgroup $P \subset \mathrm{Sp}_{2 n+2}$ containing the standard Borel subgroup $B_{2 n+2}$. Let $\operatorname{IF}^{\text {even }}:=\operatorname{IF}\left(i_{1}, \ldots, i_{r} ; 2 n+2\right)$ be the corresponding symplectic flag variety. The Schubert cells $Y(w)^{\circ}$ form a stratification of $\mathrm{IF}^{\text {even }}$, when $w$ varies in $W^{P}$. This implies that the Schubert classes $[Y(w)] \in H_{2 \ell(w)}\left(\mathrm{IF}^{\text {even }}\right)$ form a basis of the (integral) homology of IF ${ }^{e v e n}$. Since IF ${ }^{\text {even }}$ is smooth, the Schubert classes determine cohomology classes $[Y(w)] \in H^{2 \operatorname{dim} \mathrm{IF}^{e v e n}-2 \ell(w)}\left(\mathrm{IF}^{e v e n}\right)$, with the product given by intersection.

The odd-symplectic flag manifold IF $:=\operatorname{IF}\left(i_{1}, \ldots, i_{r} ; 2 n+1\right)$ is a smooth Schubert variety in $\mathrm{IF}^{\text {even }}$, therefore its Schubert classes $[X(w)]=[Y(w)] \in H_{2 \ell(w)}(\mathrm{IF})$ for

$w \in W^{P} \cap W^{2 n+1}$ form a basis for both homology and cohomology $H_{*}(\mathrm{IF})=\mathrm{H}^{*}$ (IF) with coefficients from $\mathbb{Z}$. For $\mathcal{X} \in\left\{\mathrm{IF}^{\text {even }}, \mathrm{IF}\right\}$ there is a non-degenerate Poincaré pairing $\langle\cdot, \cdot\rangle: \mathrm{H}^{*}(\mathcal{X}) \otimes \mathrm{H}^{*}(\mathcal{X}) \rightarrow \mathrm{H}^{*}(p t)$ given by

$$
\left\langle\gamma_{1}, \gamma_{2}\right\rangle=\int_{\mathcal{X}} \gamma_{1} \cup \gamma_{2}
$$

where the integral is the push-forward to the point, i.e. $\int_{\mathcal{X}} \gamma:=p_{*}(\gamma)$ and $p: \mathcal{X} \rightarrow p t$ is the structure morphism. For a cohomology class $\gamma \in \mathrm{H}^{*}(\mathcal{X})$ we denote by $\gamma^{\vee}$ its Poincaré dual. Thus $\int_{\mathrm{IF}}[X(u)] \cup[X(v)]^{\vee}=\delta_{u, v}$.

Remark 3.0.1. Although it will not be used in the sequel, we note that the Poincaré dual of a Schubert class in $\mathrm{H}^{*}\left(\mathrm{IF}^{\text {even }}\right)$ ia again a Schubert class; indeed this is true for any homogeneous space $G / P$ (see [7]). However, this is no longer true in the odd-symplectic case. Formulas for Poincaré dual classes of Schubert classes in the odd-symplectic Grassmannian IG $(k, 2 n+1)$ were calculated by Pech in [56, Prop. 3] for $k=2$ and in [55, Prop. 2.11, p.50] for arbitrary $k$.

It is customary to identify $H_{2}\left(\mathrm{IF}^{\text {even }}\right)=\mathbb{Z} \Delta^{\vee} / \mathbb{Z} \Delta_{P}^{\vee}$; see e.g. [16, §3] for precise details. Under this identification $H_{2}\left(\mathrm{IF}^{\text {even }}\right)=\oplus_{\alpha_{i} \in \Delta \backslash \Delta_{P}} \mathbb{Z}\left(\alpha_{i}^{\vee}+\Delta_{P}^{\vee}\right)$. Then the inclusion $\iota: \mathrm{IF} \rightarrow \mathrm{IF}^{\text {even }}$ gives an inclusion of abelian groups $\iota_{*}: H_{2}(\mathrm{IF}) \rightarrow H_{2}\left(\mathrm{IF}^{\text {even }}\right)$ with image spanned by those $\alpha_{i}^{\vee}+\Delta_{P}^{\vee}$ such that $s_{i} \leq w_{0}$.

We review some basic facts about the equivariant cohomology ring, focusing on $\mathrm{H}_{T}^{*}(\mathrm{IF})$. We refer to [2] for more details. For any topological space $Z$ with a left 
torus $T$ action, its equivariant cohomology ring is the ordinary cohomology of the Borel mixed space $Z_{T}:=(E T \times Z) / T$ where $E T \rightarrow B T$ is the universal $T$-bundle, and $T$ acts on $E T \times Z$ by $t \cdot(e, z)=\left(e t, t^{-1} z\right)$. In particular, $\mathrm{H}_{T}^{*}(p t)=\mathrm{H}^{*}(B T)$ is a polynomial ring $\mathbb{Z}\left[t_{1}, \ldots, t_{s}\right]$ where $t_{i}$ are an additive basis for $(\text { Lie } T)^{*}$. The continuous map $X_{T} \rightarrow B T$ gives a $\mathrm{H}_{T}^{*}(p t)$-algebra structure on $\mathrm{H}_{T}^{*}(Z)$.

Let now $Z=$ IF with its natural $T \simeq\left(\mathbb{C}^{*}\right)^{n+1}$ action. The Schubert varieties $X(u) \subset$ IF are $T$-stable, and the fundamental classes $[X(u)]_{T} \in H_{2 \ell(u)}^{T}(\mathrm{IF})$ give an $\mathrm{H}_{T}^{*}(p t)$-basis for the equivariant (co)homology $\mathrm{H}_{T}^{*}(\mathrm{IF})=H_{*}^{T}(\mathrm{IF})$. The inclusion $\iota: \mathrm{IF} \rightarrow \mathrm{IF}^{\text {even }}$ is equivariant with respect to the map $T \subset T_{2 n+2}$ and therefore there is an opposite map $\mathrm{H}_{T_{2 n+2}}^{*}\left(\mathrm{IF}^{\text {even }}\right) \rightarrow \mathrm{H}_{T}^{*}(\mathrm{IF})$ such that $\mathrm{H}_{T}^{*}(p t)=\mathrm{H}_{T_{2 n+2}}^{*}(p t)=$ $\mathbb{Z}\left[t_{1}, \ldots t_{n+1}\right]$. We use the same conventions as in $[16, \S 8]$ for the geometric interpretation of the characters $t_{i}$ inside the equivariant cohomology ring. There is an equivariant version of the Poincaré pairing $\langle\cdot, \cdot\rangle: \mathrm{H}_{T}^{*}(\mathrm{IF}) \otimes \mathrm{H}_{T}^{*}(\mathrm{IF}) \rightarrow \mathrm{H}_{T}^{*}(p t)$ given by the (equivariant) push forward map to the point:

$$
\left\langle\gamma_{1}, \gamma_{2}\right\rangle=\int_{\mathrm{IF}}^{T} \gamma_{1} \cup \gamma_{2}:=p_{*}^{T}\left(\gamma_{1} \cup \gamma_{2}\right) \in \mathrm{H}_{T}^{*}(p t) .
$$




\section{Chapter 4}

\section{(Equivariant) Quantum cohomology}

In this section we recall some basic facts about equivariant Gromov-Witten (EGW) invariants and the equivariant quantum (EQ) cohomology rings. For the purposes of this paper we specialize to the odd and even-symplectic Grassmannian case.

\subsection{Equivariant Gromov-Witten invariants}

Set $\mathrm{IG}:=\mathrm{IG}(k, 2 n+1)$ and $\mathrm{IG}^{\text {even }}:=\mathrm{IG}(k, 2 n+2)$, with $\iota: \mathrm{IG} \rightarrow \mathrm{IG}^{\text {even }}$ the natural embedding. Let $\mathcal{X} \in\left\{\mathrm{IG}, \mathrm{IG}^{\text {even }}\right\}$. Recall that $H_{2}(\mathcal{X})=\mathbb{Z}$ with the generator given by the Schubert class $\left[X\left(s_{k}\right)\right] \in H_{2}(\mathrm{IG})$ or $\left[Y\left(s_{k}\right)\right]=\iota_{*}\left[X\left(s_{k}\right)\right]$. We abuse notation and from now on we identify $\left[X\left(s_{k}\right)\right]=\left[Y\left(s_{k}\right)\right]$. A degree $d$ in $\mathcal{X}$ is an effective homology class $d \in H_{2}(\mathcal{X})$. In our situation a degree is a non-negative integer. Let $\overline{\mathcal{M}}_{0, r}(\mathcal{X}, d)$ be the Kontsevich moduli of stable maps to $\mathcal{X}$ of degree $d$ to $\mathcal{X}$ with $r$ marked points $(r \geq 0)$; see e.g. [21]. This is a projective algebraic variety of expected dimension

$$
\operatorname{expdim} \overline{\mathcal{M}}_{0, r}(\mathcal{X}, d)=\operatorname{dim} \mathcal{X}+\int_{\left[X\left(s_{k}\right)\right]} c_{1}\left(T_{\mathcal{X}}\right)+r-3
$$

where $T_{\mathcal{X}}$ denotes the tangent bundle of $\mathcal{X}$.

Lemma 4.1.1. Let $X($ Div) and $Y($ Div) be the (unique!) Schubert divisors in IG and $\mathrm{IG}^{\text {even }}$ and let $\iota: \mathrm{IG} \rightarrow \mathrm{IG}^{\text {even }}$ be the inclusion. Then the following equalities hold:

(a) $\iota^{*}[Y($ Div $)]=[X($ Div $)]$;

(b) $c_{1}\left(T_{\mathrm{IG}}\right)=(2 n+2-k)[X($ Div $)]$ and $c_{1}\left(T_{\mathrm{IG}^{\text {even }}}\right)=(2 n+3-k)[Y($ Div $)]$.

(c)

$$
\int_{\left[X\left(s_{k}\right)\right]} c_{1}\left(T_{\mathcal{X}}\right)= \begin{cases}2 n+2-k & \text { if } \mathcal{X}=\mathrm{IG} \\ 2 n+3-k & \text { if } \mathcal{X}=\mathrm{IG}^{\text {even }}\end{cases}
$$

Proof. A more general version of the first identity was proved by Pech in her thesis [55, Prop. 2.9]. The explicit calculation of the class of the tangent bundle in the even 
case can be found e.g. in [13]. In the odd case, for $k=2$, it was done by Pech [56]. In the Appendix A we deduce this formula based on a localization argument. Finally, the second equality in (c) follows because the Schubert divisor and curve are dual to each other, i.e. $\int_{\mathrm{IG}^{\text {even }}}\left[Y(\right.$ Div) $] \cap\left[X\left(s_{k}\right)\right]=1$ (see [22] for this classical fact). The first equality follows from the projection formula and part (a):

$$
\begin{aligned}
& \int_{\left[X\left(s_{k}\right)\right]} c_{1}\left(T_{\mathrm{IG}}\right)=(2 n+2-k) \int_{\mathrm{IG}} \iota^{*}[Y(\text { Div })] \cap\left[X\left(s_{k}\right)\right] \\
& =(2 n+2-k) \int_{\mathrm{IG}^{\text {even }}}[Y(\text { Div })] \cap\left[Y\left(s_{k}\right)\right]=2 n+2-k .
\end{aligned}
$$

The points of the moduli space are (equivalence classes of) stable curves $f$ : $\left(C, p t_{1}, \ldots, p t_{r}\right) \rightarrow \mathcal{X}$ of degree $d$, where $C$ is a tree of $\mathbb{P}^{1}$ 's and $p t_{i} \in C$ are nonsingular points. The moduli space $\overline{\mathcal{M}}_{0, r}(\mathcal{X}, d)$ comes equipped with $r$ evaluation $\operatorname{maps}_{i}: \overline{\mathcal{M}}_{0,3}(\mathcal{X}, d) \rightarrow \mathcal{X}$ which sends $\left(C, p t_{1}, \ldots p t_{r} ; f\right)$ to $f\left(p t_{i}\right)$. We refer e.g. to [21] for details.

Let $\gamma_{1}, \ldots, \gamma_{r} \in \mathrm{H}^{*}(\mathcal{X})$ and $d \in H_{2}(\mathcal{X})$ an effective degree. The $r$-point, genus 0 , GW invariant is defined by

$$
\left\langle\gamma_{1}, \ldots, \gamma_{r}\right\rangle_{d}:=\int_{\left[\overline{\mathcal{M}}_{0, r}(\mathcal{X}, d)\right] u^{i i r}} \operatorname{ev}_{1}^{*}\left(\gamma_{1}\right) \cup \mathrm{ev}_{2}^{*}\left(\gamma_{2}\right) \cup \ldots \cup \mathrm{ev}_{r}^{*}\left(\gamma_{r}\right),
$$

where $\left[\overline{\mathcal{M}}_{0, r}(\mathcal{X}, d)\right]^{v i r}$ is the virtual fundamental class. This is a cycle

$$
\left[\overline{\mathcal{M}}_{0, r}(\mathcal{X}, d)\right]^{v i r} \in H_{2 \operatorname{expdim}} \overline{\mathcal{M}}_{0, r}(\mathcal{X}, d)\left(\overline{\mathcal{M}}_{0, r}(\mathcal{X}, d)\right) .
$$

Givental studied equivariant Gromov-Witten invariants in [28]. If $\mathcal{X}=\mathrm{IG}^{\text {even }}$ then the moduli space $\overline{\mathcal{M}}_{0, r}(\mathcal{X}, d)$ is an irreducible algebraic variety [34,64], and the virtual fundamental class coincides to the fundamental class. This is true more generally when $\mathrm{IF}^{\text {even }}$ is replaced by any generalized flag manifold $G / P$. However, IF is not homogeneous, and this result is no longer available. In the particular case when $d=1$, Pech used obstruction theory to prove the following (cf. [55, Proposition 2.15]; for $k=2$ [56, Proposition 13]):

Proposition 4.1.2. Let $r=1,2,3$. Then the moduli space of stable maps

$$
\overline{\mathcal{M}}_{0, r}(\operatorname{IG}(k, 2 n+1), 1)
$$

is a smooth, irreducible, algebraic variety of complex dimension $k(2 n+1-k)-\frac{k(k-1)}{2}+$ $(2 n+2-k)+r-3$.

The GW invariants satisfy the "divisor axiom" property: if $[D] \in H^{2}(\mathcal{X})$ is the class corresponding to a complex subvariety of codimension 1 then for any $\gamma_{2}, \ldots, \gamma_{r} \in$ $\mathrm{H}^{*}(\mathcal{X})$

$$
\left\langle[D], \gamma_{2}, \ldots, \gamma_{r}\right\rangle_{d}=([D] \cap d)\left\langle\gamma_{2}, \ldots, \gamma_{r}\right\rangle_{d} .
$$


If a group $G$ acts on $\mathcal{X}$, it determines a natural action on the moduli space $\overline{\mathcal{M}}_{0, r}(\mathcal{X}, d)$ by acting on the target of a stable map. With respect to this action, the evaluation maps $\mathrm{ev}_{i}$ are $G$-equivariant. Then the definition of the GW invariants extends to the equivariant setting, using the equivariant cohomology classes, the equivariant virtual fundamental class [35] and the equivariant push-forward to a point. The divisor axiom (4.1) remains unchanged. In this paper we will consider the give formulas calculating the $\mathrm{GW}$ invariants equivariant with respect to $G=T$, the torus acting on IG. In this case the equivariant GW invariants have values in $\mathrm{H}_{T}^{*}(p t)$.

\subsection{The (equivariant) quantum cohomology ring}

The quantum cohomology $\mathrm{QH}^{*}(\mathrm{IG})$ of $\mathrm{IG}:=\mathrm{IG}(k, 2 n+1)$ is a graded $\mathbb{Z}[q]$-algebra with a $\mathbb{Z}[q]$-basis given by Schubert classes $[X(u)]$, where $u \in W^{P}$. The multiplication is given by

$$
[X(u)] \star[X(v)]=\sum_{d \geq 0 ; w \in W^{P}} q^{d} c_{u, v}^{w, d}[X(w)],
$$

where $c_{u, v}^{w, d}=\left\langle[X(u)],[X(v)],[X(w)]^{\vee}\right\rangle_{d}$ is the GW invariant. For a smooth variety $X$ the multiplication of $\mathrm{QH}^{*}(X)$ was proved to be associative in $[38,39]$ (the sources $[21,59]$ also contains details). The degree of $q$ is

$$
\operatorname{deg} q=\int_{[X(D i v)]^{\vee}} c_{1}\left(T_{\mathrm{IG}(k, 2 n+1)}\right)=2 n+2-k,
$$

by Lemma 4.1.1 above. The grading is equivalent to the requirement that

$$
\operatorname{codim} X(u)+\operatorname{codim} X(v)=\operatorname{codim} X(w)+d \cdot \operatorname{deg} q,
$$

which in turn is equivalent the requirement that the $\mathrm{GW}$ invariant

$$
\left\langle[X(u)],[X(v)],[X(w)]^{\vee}\right\rangle_{d}
$$

is non-zero. From definition, the quantum cohomology ring is a deformation of the ordinary cohomology ring, in the sense that if one makes $q=0$ then one recovers the multiplication in $\mathrm{H}^{*}(\mathrm{IG})$. In the case when $k=n+1, \mathrm{IG}=\mathrm{LG}(n, 2 n)$ is a homogenous space (the Lagrangian Grassmannian), and a Pieri formula, calculating the quantum multiplication of a Schubert class by an algebra generator has been obtained in $[15,37]$. In the "odd" case, for $k=2$, a Pieri formula has been obtained by Pech [56]. It is the main goal of this paper to give algorithms to calculate quantum multiplication for arbitrary $k$.

Remark 4.2.1. A consequence of the Kleiman-Bertini theorem shows that the GW invariants for homogeneous spaces are enumerative $[21,36]$. This is no longer true for odd-symplectic flag manifolds. For example, Pech proved that in $\mathrm{QH}^{*}(\operatorname{IG}(2,5))$,

$$
[X(3,-1)] \star[X(2,1)]=-1[X(3,2)]+\ldots ; \quad[X(3,-1)] \star[X(3,-1)]=-q[X(0)]+\ldots
$$


As before there is an equivariant version of the quantum cohomology ring, denoted $\mathrm{QH}_{T}^{*}(\mathrm{IG})$, which deforms the multiplication in $\mathrm{H}_{T}^{*}(\mathrm{IG})$. The equivariant version of quantum cohomology was introduced by Givental and Kim as an additional tool to understand quantum cohomology $[27,35]$. This is a graded, free algebra over $\mathrm{H}_{T}^{*}(p t)[q]$ with a basis given by equivariant Schubert classes $[X(u)]_{T}$, where $u$ varies in $W^{P}$. The multiplication is defined in the same way, using the equivariant GW invariants. The structure constants $c_{u, v}^{w, d} \in \mathrm{H}_{T}^{*}(p t)$ are homogeneous polynomials of degree

$$
\operatorname{deg} c_{u, v}^{w, d}=\operatorname{codim} X(u)+\operatorname{codim} X(v)-\operatorname{codim} X(w)-d \cdot \operatorname{deg} q .
$$

If this degree equals 0 , then one recovers the structure constant from the ordinary (non-equivariant) quantum cohomology ring. 


\section{Chapter 5}

\section{The moment graphs of even and odd symplectic flag manifolds}

Sometimes called the GKM graph, the moment graph of a variety $X$ with a torus $T$ action encodes the $T$-fixed loci of $X$ together with the 1-dimensional torus orbits. In our situation $X$ will be a symplectic flag manifold or one of its Schubert varieties. The description of the moment graphs in these cases is a standard tool, and it can be found e.g in $[20,29]$

For now let $\mathrm{IF}^{\text {even }}:=\operatorname{IF}\left(i_{1}, \ldots, i_{r} ; 2 n+2\right)$ be the symplectic flag manifold corresponding to the parabolic subgroup $P:=P_{\left\{i_{1}, \ldots, i_{r}\right\}}$ containing the torus $T_{2 n+2}$. The $T_{2 n+2}$-fixed points are in bijection to the cosets in $W / W_{P}$, thus to the minimal length representatives $w \in W^{P}$. For each signed permutation $\left(w(1)<\cdots<w\left(i_{1}\right) \mid w\left(i_{1}+1\right)<\right.$ $\left.\cdots<w\left(i_{2}\right)|\cdots| w\left(i_{r-1}+1\right)<\cdots<w\left(i_{r}\right) \mid w_{i_{r+1}}<\cdots<w(n+1)\right) \in W^{P}$ the corresponding torus fixed point is the partial flag

$$
\mathbf{e}_{w}:=\left(\left\langle\mathbf{e}_{w(1)}, \ldots, \mathbf{e}_{w\left(i_{1}\right)}\right\rangle \subset\left\langle\mathbf{e}_{w(1)}, \ldots, \mathbf{e}_{w\left(i_{2}\right)}\right\rangle \subset \cdots \subset \mathbb{C}^{2 n+2}\right) .
$$

The irreducible $T_{2 n+2}$-stable curves are of the form $C_{\alpha}(w) \simeq \mathbb{P}^{1}$ which joins two fixed points $\mathbf{e}_{w}$ and $\mathbf{e}_{w s_{\alpha}}$ where $\alpha \in R^{+} \backslash R_{P}^{+}$is a positive root not in $P$. Recall, under the natural identification $H_{2}\left(\mathrm{IF}^{\text {even }}\right)$ to $\mathbb{Z} \Delta^{\vee} / \mathbb{Z} \Delta_{P}^{\vee}$, the homology class $\left[C_{\alpha}(w)\right]=$ $\alpha^{\vee}+\Delta_{P}^{\vee}$.

The moment graph of $\mathrm{IF}^{\text {even }}$ has a set of vertices indexed by $w \in W^{P}$ and edges of the form $w \longrightarrow w s_{\alpha}$ for $\alpha \in R^{+} \backslash R_{P}^{+}$. Geometrically, the vertex $w$ corresponds to the $T_{2 n+2}$-fixed point $\mathbf{e}_{w}$ and an edge $w \longrightarrow w s_{\alpha}$ to the torus stable curve $C_{\alpha}(w)$.

Let IF $:=\operatorname{IF}\left(i_{1}, \ldots, i_{r} ; 2 n+1\right)$ be the odd-symplectic flag variety, which is a Schubert subvariety of $\mathrm{IF}^{\text {even }}$. It follows that with respect to the $T_{2 n+2}$-action, the moment graph of IF is a (full) subgraph of that for IF ${ }^{\text {even }}$, with vertices corresponding to permutations $w \in W^{2 n+1} \cap W^{P}$, i.e. those minimal length representatives $w \leq w_{0}$. Recall that the odd-symplectic torus $T \subset \mathrm{Sp}_{2 n+1}$ is obtained by restricting the "even" torus $T_{2 n+2}$. The quotient $T_{2 n+2} / T$ acts trivially on IF, which implies that the moment graph of IF with respect to $T$ coincides with the one for $T_{2 n+2}$. (This was also noticed in Mihai's thesis [47, Ex. 5.2.2 and Ex. 5.2.8].) From now on we will not differentiate between the moment graphs if IF for $T$ or $T_{2 n+2}$. 


\subsection{The Grassmannian case}

Next we will give a more explicit description of the moment graphs for $\mathrm{IG}(k, 2 n+1) \subset$ $\mathrm{IG}(k, 2 n+2)$, for $k<n+1$. Recall that the corresponding subset of simple roots is $\Delta_{P_{k}}:=\Delta \backslash\left\{\alpha_{k}=t_{k}-t_{k+1}\right\}$ and that $H_{2}(\operatorname{IG}(k, 2 n+1))$ is one dimensional with generator $\alpha_{k}^{\vee}+\mathbb{Z} \Delta_{P_{k}}^{\vee}$. The edges of the moment graph correspond to $T_{2 n+2}$-stable curves of the form $C_{\alpha}(w)$, where $w \in W^{P_{k}}$ and

$\alpha \in R^{+} \backslash R_{P_{k}}^{+}=\left\{t_{i}-t_{j}: 1 \leq i \leq k<j \leq n+1\right\} \cup\left\{t_{i}+t_{j}, 2 t_{i}: 1 \leq i<j \leq n+1, i \leq k\right\}$.

The coefficients of $\alpha_{k}^{\vee}$ in the expansion of $\alpha^{\vee}$ in terms of simple coroots, and the degree of the corresponding curves, are calculated as follows:

- $\alpha^{\vee}=t_{i}-t_{j}=\left(t_{i}-t_{i+1}\right)+\cdots+\left(t_{j-1}-t_{j}\right)$; in this case the coefficient of $\alpha_{k}^{\vee}$, thus the degree of the curve $C_{\alpha}(w)$ is equal to 1 ;

- $\alpha^{\vee}=t_{i}=\left(t_{i}-t_{i+1}\right)+\left(t_{i+1}-t_{i+2}\right)+\cdots+\left(t_{n}-t_{n+1}\right)+t_{n+1}$; in this case $C_{\alpha}(w)$ has degree 1 ;

- if $j<n+1$ then $t_{i}+t_{j}=\left(t_{i}-t_{i+1}\right)+\cdots+\left(t_{j-1}-t_{j}\right)+2\left(t_{j}-t_{j+1}\right)+\cdots+2\left(t_{n-1}-\right.$ $\left.t_{n}\right)+2 t_{n+1}$. If $j=n+1$ then $t_{i}+t_{j}=\left(t_{i}-t_{i+1}\right)+\cdots+\left(t_{n}-t_{n+1}\right)+2 t_{n+1}$. Therefore the curve $C_{\alpha}(w)$ has degree 1 if $j>k$ and degree 2 if $j \leq k$.

The minimal length representatives in $w \in W^{P_{k}}$ are in one to one correspondence to sequences $1 \leq w(1)<\ldots<w(k) \leq \overline{1}$. Those corresponding to the odd-symplectic Grassmannian satisfy in addition $w(k) \leq \overline{2}$. Because it will be repeatedly used later on, we list below the vertices adjacent to the identity element in the moment graph of $\mathrm{IG}(k, 2 n+2)$, together with the degrees of the corresponding curves. Recall the convention $\bar{s}=2 n+3-s$. For now we let $k>1$.

(i) $(1<2<\cdots<i-1<i+1<\cdots<k<j)$ where $k<j \leq n+1$;

(ii) $(1<2<\cdots<i-1<i+1<\cdots<k<\bar{j})$ where $n+1<\bar{j} \leq \overline{k+1}$;

(iii) $(1<2<\cdots<i-1<i+1<\cdots<j-1<j+1<\cdots<k<\bar{j}<\bar{i})$;

(iv) $(1<2<\cdots<i-1<i+1<\cdots<k<\bar{i})$.

The edge in (i) corresponds to $\alpha=t_{i}-t_{j}$, those in (ii) and (iii) to $\alpha=t_{i}+t_{j}$ and that in (iv) to $\alpha=2 t_{i}$. In particular, only the edge in (iii) has degree 2 , and the others have degree 1 . If $k=1$, the case (iii) does not apply, and the remaining vertices in cases (i), (ii) and (iv) are respectively $(j),(\bar{j})$ and $(\overline{1})$.

The figure below illustrates the moment graphs of $\operatorname{IG}(2,5)$ and $\operatorname{IG}(2,6)$. 
Figure 5.1: The figure is the moment graph of $\operatorname{IG}(2,6)$ without degree labels. The blue vertices and edges are not included in the Schubert variety $\operatorname{IG}(2,5)$. Also, the red vertices and edges are in the closed orbit which is isomorphic to $\operatorname{IG}(1,4)$.

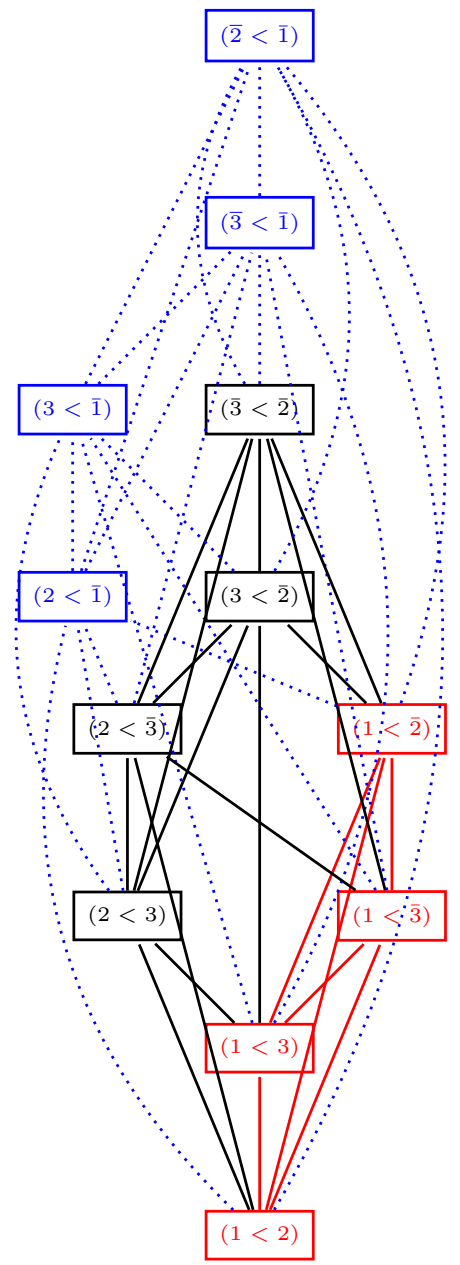




\section{Chapter 6}

\section{Curve neighborhoods}

As before let $\mathcal{X} \in\left\{\mathrm{IG}^{\text {even }}:=\mathrm{IG}(k, 2 n+2), \mathrm{IG}:=\mathrm{IG}(k, 2 n+1)\right\}$, let $d \in H_{2}(\mathcal{X})$ an effective degree, and let $\Omega \subset \mathcal{X}$ a closed subvariety. Consider the moduli space of stable maps $\overline{\mathcal{M}}_{0,2}(\mathcal{X}, d)$ with evaluation maps ev ${ }_{1}, \mathrm{ev}_{2}$. The curve neighborhood of $\Omega$ is the subscheme

$$
\Gamma_{d}(\Omega):=\mathrm{ev}_{2}\left(\mathrm{ev}_{1}^{-1} \Omega\right) \subset \mathcal{X}
$$

endowed with the reduced scheme structure. This notion was introduced by Buch, Chaput, Mihalcea and Perrin [9] to help study the quantum K theory ring of cominuscule Grassmannians. It was analyzed further for any homogeneous space by Buch and Mihalcea [16], in relation to 2-point K-theoretic GW invariants, and to a new proof of the quantum Chevalley formula in quantum cohomology. A description of the curve neighborhoods of Richardson varieties where also found in [43] when certain conditions are applied. Finally it appears the technique of curve neighborhoods may apply to finding a Chevalley formula for the quantum cohomology of weighted Grassmannians [19]. Often, estimates for the dimension of the curve neighborhoods provide vanishing conditions for certain GW invariants. In this paper we will use this technique to prove vanishing of "Chevalley" GW invariants of degree $d \geq 2$ in IG.

We start with the observation (going back to [9]) that if $\Omega$ is a Schubert variety, then $\Gamma_{d}(\Omega)$ must be a (finite) union of Schubert varieties, stable under the same Borel subgroup. This follows because $\Omega$ is stable under the appropriate Borel subgroup, and $\mathrm{ev}_{1}, \mathrm{ev}_{2}$ are proper, equivariant maps; thus $\Gamma_{d}(\Omega)$ is closed and Borel-equivariant.

In what follows we give estimates for the dimension of the curve neighborhoods of Schubert varieties $X(w) \subset \mathrm{IG}$, using known estimates for the dimension in the even case.

\subsection{Curve neighborhoods of Schubert varieties in $\mathrm{IG}^{\text {even }}$}

Let $P:=P_{k}$ be the maximal parabolic subgroup such that $\mathrm{Sp}_{2 n+2} / P=\mathrm{IG}^{\text {even }}$. Let $w \in W^{P}$ be a minimal length representative and $d \in H_{2}\left(\mathrm{IG}^{\text {even }}\right)$. Recall that it 
was proved in [9] that the curve neighborhood $\Gamma_{d}(Y(w))$ must be a Schubert variety. Further, it follows from [16] that $\Gamma_{d}(Y(w))=Y\left(w \cdot z_{d} W_{P}\right)$, where $z_{d} \in W$ is defined by the condition that $\Gamma_{d}(1 . P)=Y\left(z_{d} W_{P}\right)$. In [16] it was found a recursive formula for $z_{d}$, which we recall next.

Let $q_{2 n+2}$ be the quantum parameter for $\mathrm{QH}^{*}\left(\mathrm{IG}^{\text {even }}\right)$. Lemma 4.1 .1 implies that this is an indeterminate of degree $2 n+3-k$. The maximal elements of the set $\{\beta \in$ $\left.R^{+} \backslash R_{P}^{+}: \alpha^{\vee}+\Delta_{P} \leq d\right\}$ are called maximal roots of $d$. The root $\beta \in R^{+} \backslash R_{P}^{+}$is called $P$-cosmall if $\beta$ is a maximal root of $\beta^{\vee}+\Delta_{P}^{\vee} \in H_{2}\left(\mathrm{IG}^{\text {even }}\right)$. In type $C_{n+1}, P$-cosmall roots are among the roots $2 t_{i}$ for $1 \leq i \leq n+1$, and $t_{i}-t_{j}$ for $1 \leq i<j \leq n+1$. The following foillows from [16, Corollary 4.12, Theorem 6.2, Theorem 5.1, and Theorem $7.2]$.

Proposition 6.1.1. Let $d \in H_{2}\left(\mathrm{IG}^{\text {even }}\right)$ be an effective degree and $w \in W^{P}$. Then the following hold:

1. If $\alpha \in R^{+}-R_{P_{k}}^{+}$is a maximal root of $d$, then $s_{\alpha} \cdot z_{d-\alpha^{\vee}} W_{P}=z_{d} W_{P}$;

2. $\operatorname{dim} \Gamma_{d}(Y(w)) \leq \ell(w)+\ell\left(z_{d} W_{P}\right) \leq \ell(w)+\operatorname{deg} q_{2 n+2}^{d}-1$. Furthermore, if the second equality occurs then $d=\alpha^{\vee}+\mathbb{Z} \Delta_{P_{k}}^{\vee}$ and $\alpha$ is a $P$-cosmall root.

Corollary 6.1.2. (a) There is an equality $z_{1} W_{P}=s_{2 t_{1}} W_{P}$ and the minimal length representative of $z_{1} W_{P}$ is $(2<3<\cdots<k<\overline{1})$.

(b) There is an inequality $\ell\left(z_{d} W_{P}\right) \leq d \operatorname{deg} q_{2 n+2}-1$ with equality if and only if $d=1$.

(c) If $k>1$ and $d=2$ then $z_{2} W_{P}=s_{t_{1}+t_{2}} W_{P}$ and $\ell\left(z_{2} W_{P}\right)=2 \operatorname{deg} q_{2 n+2}-3$.

(d) If $k=1$ then $z_{1} W_{P}=z_{2} W_{P}$ and $\ell\left(z_{2} W_{P}\right)=2 n+1<2 \operatorname{deg} q_{2 n+2}-3$.

Proof. The first part follows directly from the part (1) of the proposition. The equality in (b) follows by direct calculation of $\ell\left(s_{2 t_{1}} W_{P}\right)$, using its minimal length representative. Using the calculation of degrees associated to roots from section 5.1 we deduce that no degree $d \geq 2$ can be the degree of a cosmall root, thus equality cannot occur in this case.

For part (c), notice that since $2 t_{1}$ is a maximal root of 1 , therefore $z_{1} W_{P}=s_{2 t_{1}} W_{P}$. By employing the recursion in Proposition 6.1.1 we obtain $z_{2} W_{P}=s_{2 t_{1}} \cdot s_{2 t_{1}} W_{P}$. Now observe the following:

$$
\begin{aligned}
s_{2 t_{1}} \cdot s_{2 t_{1}} & =\left(s_{1} \ldots s_{n+1} \ldots s_{1}\right) \cdot\left(s_{1} \ldots s_{n+1} \ldots s_{1}\right) \\
& =s_{1} \cdot s_{2 t_{2}} \cdot s_{1} \cdot s_{2 t_{2}} \cdot s_{1} \\
& =s_{1} \cdot s_{t_{1}+t_{2}} \cdot s_{1} \\
& =s_{t_{1}+t_{2}} \cdot s_{1} \cdot s_{1} \\
& =s_{t_{1}+t_{2}} \cdot s_{1} .
\end{aligned}
$$

Since $s_{1} \in W_{P}$, the above shows that $z_{2} W_{P}=s_{t_{1}+t_{2}} W_{P}$ as claimed. The "inequality" $\ell\left(z_{2} W_{P}\right)$ " $\leq " 2 \operatorname{deg} q_{2 n+2}-3$ follows by a direct calculation, using the minimal length representative of $s_{t_{1}+t_{2}} W_{P}$. Finally, part $(d)$ follows from the observation that if $k=1$, 
then $\operatorname{IG}(1,2 n+1)=\mathbb{P}^{2 n}$, and then $\Gamma_{1}(i d)=\mathbb{P}^{2 n}$, thus $\ell\left(z_{1} W_{P}\right)=\operatorname{dim} \operatorname{IG}(1,2 n+$ 1).

We will need the following lemma.

Lemma 6.1.3. Let $w=(w(1)<w(2)<\cdots<w(k)) \in W^{P}$. Then $w \cdot z_{1}$ is reduced and a minimal length representative in $W^{P}$ if and only if $w(1)=1$. In particular, $\operatorname{dim} \Gamma_{1}(Y(w))=\ell(w)+\operatorname{deg} q_{2 n+2}-1$ if and only if $Y(w) \subset X_{c}$ is a Schubert variety in the closed orbit of IG.

Proof. Using the bijection between $W^{P}$ and $\Lambda^{2 n+2}$ and that fact that codim $Y(\lambda)=$ $|\lambda|$ we have that

$$
\begin{aligned}
\ell\left(w z_{1}\right)-\ell(w) & =\operatorname{codim} Y(w)-\operatorname{codim} Y\left(w z_{1}\right) \\
& =2 n+4-2 w_{1}-k+\#\left\{j: w_{1}+w_{j}>2 n+3\right\}
\end{aligned}
$$

So the following equations are equivalent.

$$
\begin{aligned}
\ell\left(w z_{1}\right)-\ell(w) & =\ell\left(z_{1}\right) \\
2 n+4-2 w(1)-k+\#\{j: w(1)+w(j)>2 n+3\} & =2 n+2-k \\
2-2 w(1)+\#\{j: w(1)+w(j)>2 n+3\} & =0 \\
\#\{j: w(1)+w(j)>2 n+3\} & =2 w(1)-2
\end{aligned}
$$

The equality implies $w(1)=1$.

For the reverse direction let $w(1)=1$. Observe it is sufficient to check $w z_{1}$ is a minimal length representative by the equivalent equations in the first part of the proof. Let $w=(1<w(2)<\cdots<w(k) \mid w(k+1)<\cdots<w(n+1))$ and recall $z_{1}=(2<3<\cdots<k<\overline{1} \mid(k+1)<\cdots<(n+1))$. The multiplication $w z_{1}$ in $W$ where the permutations are in one line notation is

$$
\begin{aligned}
(1 w(2) \cdots w(k) w(k+1) \cdots w(n+1)) & *(23 \cdots k \overline{1}(k+1) \cdots(n+1)) \\
= & w(2) w(3) \cdots w(k) \overline{1} w(k+1) \cdots w(n+1) .
\end{aligned}
$$

This direct calculation that shows $w z_{1}$ is a minimal length representative. The result follows.

\subsection{Curve neighborhoods for IG.}

Let $w \in W^{P} \cap W^{2 n+1}$ and let $d \in H_{2}(\mathrm{IG})$ be an effective degree. As mentioned above, the curve neighborhood $\Gamma_{d}(X(w))$ of $X(w)$ is a closed, $B$-stable subvariety of IG, therefore it must be an union of Schubert varieties:

$$
\Gamma_{d}(X(w))=X\left(w^{1}\right) \cup \cdots \cup X\left(w^{r}\right)
$$

where $w^{i} \in W^{P} \cap W^{2 n+1}$. As noticed in [16] or [44], the permutations $w^{i}$ can be determined combinatorially from the moment graph. 
Proposition 6.2.1. Let $w \in W^{P} \cap W^{2 n+1}$. in the moment graph of IG, let $\left\{v^{1}, \cdots, v^{s}\right\}$ be the maximal vertices in the moment graph which can be reached from any $u \leq w$ using a path of degree $d$ or less. Then $\Gamma_{d}(X(w))=X\left(v^{1}\right) \cup \cdots \cup X\left(v^{s}\right)$.

Proof. Let $Z_{w, d}=X\left(v^{1}\right) \cup \cdots \cup X\left(v^{s}\right)$. Let $v:=v^{i} \in Z_{w, d}$ be one of the maximal $T$-fixed points. By the definition of $v^{i}$ 's and the moment graph there exists a chain of $T$-stable rational curves of degree less than or equal to $d$ joining $u \leq w$ to $v$. It follows that $v \in \Gamma_{d}(X(w))$, thus $X(v) \subset \Gamma_{d}(X(w))$, whence $Z_{w, d} \subset \Gamma_{d}(X(w))$.

For the converse inclusion, let $v \in \Gamma_{d}(X(w))$ be a $T$-fixed point. By [44, Lemma 5.3] there exists a $T$-stable curve joining a fixed point $u \in X(w)$ to $v$. This curve corresponds to a path in the moment graph of IG, thus $v \in Z_{w, d}$. Since Bruhat order is compatible with inclusion of Schubert variaties, this completes the proof.

In what follows we will obtain estimates for the dimension of the curve neighborhood $\Gamma_{d}(X(w))$, using estimates obtained in the even case. We start with the observation that the "odd" curve neighborhoods are proper subvarieties of the "even" ones.

Lemma 6.2.2. Let $w \in W^{P} \cap W^{2 n+1}$ and $d \geq 1$ an effective degree. Then there is a strict inclusion $\Gamma_{d}(X(w)) \subsetneq \Gamma_{d}(Y(w))$.

Proof. Consider the identity 1.P $\in \Gamma_{d}(X(w))$. There is a $T_{2 n+2}$-stable degree 1 curve (i.e. a line $)$ in $\operatorname{IG}(k, 2 n+2)$ that contains the $T_{2 n+2}$-fixed points $1 . P$ and $(2<$ $3<\cdots<\overline{1}) \in \mathrm{IG}^{e v e n} \backslash \mathrm{IG}$. Therefore $\Gamma_{1}(X(w)) \subsetneq \Gamma_{1}(Y(w))$, and this implies the lemma.

Part (b) of the next result is the key technical requirement needed for the vanishing of all Chevalley GW invariants.

Theorem 6.2.3. Let $w \in W^{2 n+1}$. Then the following inequalities hold:

$$
\begin{aligned}
& \operatorname{dim} \Gamma_{1}(X(w))-\operatorname{dim} X(w) \leq \operatorname{deg} q-1 \\
& \operatorname{dim} \Gamma_{d}(X(w))-\operatorname{dim} X(w)<\operatorname{deg} q^{d}-1 \text { for all } d \geq 2
\end{aligned}
$$

Further, if the Schubert variety $X(w)$ is not contained in the closed orbit $X_{c}$ of IG then

$$
\operatorname{dim} \Gamma_{1}(X(w))-\operatorname{dim} X(w)<\operatorname{deg} q-1 .
$$

Proof. Recall that $\operatorname{deg} q_{2 n+2}=\operatorname{deg} q+1$. We consider 1 the cases when $d=1$ and $d \geq 2$. If $d=1$, Lemma 6.2.2 and Proposition 6.1.1 imply that

$$
\operatorname{dim} \Gamma_{1}(X(w))+1-\operatorname{dim} X(w) \leq \operatorname{dim} \Gamma_{1}(Y(w))-\operatorname{dim} Y(w) \leq \operatorname{deg} q_{2 n+2}-1
$$

thus $\operatorname{dim} \Gamma_{1}(X(w))-\operatorname{dim} X(w) \leq \operatorname{deg} q_{2 n+2}-2=\operatorname{deg} q-1$. 
Let now $d \geq 2$, and let $\Gamma_{d}(X(w))=X\left(v^{1}\right) \cup \cdots \cup X\left(v^{s}\right)$. Then each $v^{i}$ is joined to some $u^{i} \leq w$ in the moment graph of IG by $j$ edges of degrees $d_{i} \in\{1,2\}$, where $\sum_{i=1}^{j} d_{i} \leq d$. By applying repeatedly the estimate for $d=1$, we have

$$
\operatorname{dim} X\left(v^{i}\right)-\operatorname{dim} X(w) \leq \operatorname{dim} X\left(v^{i}\right)-\operatorname{dim} X\left(u^{i}\right) \leq \sum_{i=1}^{j}\left(d_{i} \operatorname{deg} q-1\right) \leq d \operatorname{deg} q-j .
$$

If $j \geq 2$ then the result holds. If $j=1$ then necessarily $d=2$. If $k=1$ then there are no edges of degree 2 , thus $j \geq 2$ and the previous proof applies. If $k>1$, by Corollary 6.1.2(c)

$$
\begin{array}{r}
\operatorname{dim} X\left(v^{i}\right)-\operatorname{dim} X(w) \leq \operatorname{dim} \Gamma_{2}(Y(w))-1-\ell(w) \\
\leq \ell\left(z_{2} W_{P}\right)-1 \leq 2 \operatorname{deg} q_{2 n+2}-4<2 \operatorname{deg} q-1 .
\end{array}
$$

This proves the first two inequalities. For the last inequality, we notice that the hypothesis implies that $w$ is determined by a sequence $(w(1)<\cdots<w(k))$ such that $w(1)>1$. Then by Lemma 6.1 .3 combined with Proposition 6.1.1 we obtain

$\operatorname{dim} \Gamma_{1}(X(w))-\operatorname{dim} X(w) \leq \operatorname{dim} \Gamma_{1}^{\mathrm{IG}}(Y(w))-1-\ell(w)<\operatorname{deg} q_{2 n+2}-2=\operatorname{deg} q-1$

This finishes the proof. 


\section{Chapter 7}

\section{Vanishing of Chevalley Gromov-Witten invariants}

The main result of this Chapter is the following.

Theorem 7.0.1. Let $d \geq 1$ be a degree in $H_{2}(\operatorname{IG}(k, 2 n+1))$. Let $X(v), X(w) \subset$ $\mathrm{IG}(k, 2 n+1)$ be two Schubert varieties and $X($ Div $)$ the Schubert divisor. If

$$
\operatorname{dim} \Gamma_{d}(X(v))<\ell(v)+\operatorname{deg} q^{d}-1
$$

then the equivariant $G W$ invariant

$$
\left\langle[X(D i v)]_{T},[X(v)]_{T},[X(w)]_{T}^{\vee}\right\rangle_{d}=0 .
$$

In particular, the equivariant Gromov-Witten invariant above vanishes if either $d \geq 2$ or $d=1$ and $X(v)$ is not included in the closed orbit $X_{c} \subset \operatorname{IG}(k, 2 n+1)$.

Proof. By the divisor axiom

$$
\left\langle[X(\operatorname{Div})]_{T},[X(v)]_{T},[X(w)]_{T}^{\vee}\right\rangle_{d}=d\left\langle[X(v)]_{T},[X(w)]_{T}^{\vee}\right\rangle_{d}
$$

By definition,

$$
\begin{aligned}
& \left\langle[X(v)]_{T},[X(w)]_{T}^{\vee}\right\rangle_{d}=\int_{\left[\overline{\mathcal{M}}_{0,2}(\operatorname{IG}(k, 2 n+1), d)\right]^{v i r}}^{T} \operatorname{ev}_{1}^{*}[X(v)]_{T} \cup \mathrm{ev}_{2}^{*}[X(w)]_{T}^{\vee} \\
= & \int_{\mathrm{IG}(k, 2 n+1)}^{T}[X(w)]_{T}^{\vee} \cap\left(\operatorname{ev}_{2}\right)_{*}\left(\operatorname{ev}_{1}^{*}[X(v)]_{T} \cap\left[\overline{\mathcal{M}}_{0,2}(\operatorname{IG}(k, 2 n+1), d)\right]^{v i r}\right) .
\end{aligned}
$$

The cycle $\left(\mathrm{ev}_{2}\right)\left(\mathrm{ev}_{1}^{-1}[X(v)]\right)$ is supported on the curve neighborhood $\Gamma_{d}(X(v))$, and the push-forward $\left(\mathrm{ev}_{2}\right)_{*}\left(\operatorname{ev}_{1}^{*}[X(v)]_{T} \cap\left[\overline{\mathcal{M}}_{0,2}(\mathrm{IG}(k, 2 n+1), d)\right]^{v i r}\right)$ is non-zero only if the curve neighborhood has components of dimension

$$
\operatorname{expdim} \overline{\mathcal{M}}_{0,2}(\operatorname{IG}(k, 2 n+1))-\operatorname{codim} X(v)=\operatorname{deg} q^{d}-1+\ell(v) .
$$

However, the hypothesis implies that $\operatorname{dim} \Gamma_{d}(X(v))$ is strictly less than this quantity. Finally, the statement on GW invariants of degree $d \geq 2$ follows from Theorem 6.2.3. 


\section{Chapter 8}

\section{Lines in IG}

Let $X:=\operatorname{IG}(k, 2 n+1)$. According to Theorem 7.0.1, the only equivariant GW invariants $\left\langle[X(D i v)]_{T},[X(v)]_{T},[X(w)]_{T}^{\vee}\right\rangle_{d}$ which are non-zero are those when $d=1$ and the Schubert variety $X(v)$ is included in the closed orbit $X_{c} \simeq \operatorname{IG}(k-1,2 n)$. We will need a separate argument to calculate these invariants. For that, we will analyze some geometric properties of the moduli spaces of stable maps $\overline{\mathcal{M}}_{0, r}(\mathrm{IG}, 1) \rightarrow$ IG where $r=1,2$ and the Gromov-Witten varieties

$$
G W_{1}(w):=\mathrm{ev}_{1}^{-1}(X(w)) \subset \overline{\mathcal{M}}_{0,2}(\mathrm{IG}, 1) .
$$

For $X(w) \subset X_{c}$, we will show that $G W_{1}(w)$ is a reduced algebraic scheme which has 2 irreducible components, one corresponding to lines in IG contained in the closed orbit $X_{c}$, and another containing lines which intersect the open orbot $X^{\circ}$. The restriction of the evaluation maps to each of these components will be a surjective map, which is either birational, or it has general fiber of positive dimension. We will deduce from this that the curve neighborhood $\Gamma_{1}(X(w))$ has two components, and that if non-zero, the GW invariant is equal to 1 precisely in the cases when $[X(w)]^{\vee}$ is Poincaré dual to one of these components.

From now on, a line in $X$ will mean an irreducible, reduced, curve of degree 1. Recall that there is a sequence of embeddings

$$
X=\mathrm{IG}(k, 2 n+1) \subset \mathrm{IG}(k, 2 n+2) \subset \mathrm{Gr}(k, 2 n+2) \subset \mathbb{P}\left(\bigwedge^{k} \mathbb{C}^{2 n+2}\right)
$$

where the last is the Plücker embedding. The image of a line in IG under the composition of these embeddings is a projective line. Indeed, a calculation in coordinates shows that the image of the Schubert curve in $X$ is the Schubert curve in $\operatorname{Gr}(k, 2 n+2)$, and the image of this Schubert curve is a projective line (see Appendix B). We record the following consequence:

Let $r=1,2,3$. Recall from Prop. 4.1 .2 above that Pech proved that $\overline{\mathcal{M}}_{0, r}(X, 1)$ is a non-singular, irreducible scheme of dimension

$\operatorname{dim} \overline{\mathcal{M}}_{0, r}(X, 1)=\operatorname{dim} X+\operatorname{deg} q+r-3=k(2 n+1-k)-\frac{k(k-1)}{2}+2 n+2-k+r-3$. 
In what follows we will identify $\overline{\mathcal{M}}_{0,1}(X, 1)$ with a (necessarily smooth) subvariety of the ordinary flag manifold $\mathrm{Fl}(k-1, k, k+1 ; 2 n+1)$.

There is a natural isomorphism $\overline{\mathcal{M}}_{0,1}(\operatorname{Gr}(k, 2 n+1), 1) \simeq \mathrm{Fl}(k-1, k, k+1 ; 2 n+1)$ such that the evaluation map ev $\mathrm{v}_{1}$ is the projection $\pi_{k}: \mathrm{Fl}(k-1, k, k+1 ; 2 n+1) \rightarrow$ $\operatorname{Gr}(k, 2 n+1)$. To see the isomorphism explicitly, one can use e.g. the kernel-span technique of Buch [10] to observe that to any line $L \subset$ Gr one can associate its kernel $K:=\bigcap_{V \in L} V$ and its span $S:=\operatorname{Span}\{V: V \in L\}$, which have dimension $k-1$, respectively $k+1$. Then the pointed line $(p \in L)$ is sent to $(\operatorname{ker} L, p$, Span $L)$. We would like to identify $\overline{\mathcal{M}}_{0,1}(X, 1)$ to a subvariety of the three-step flag variety. The idea is that if $V_{k-1} \in \operatorname{IG}(k-1,2 n+1)$ then a triple $\left(V_{k-1} \subset V_{k} \subset V_{k+1}\right)$ corresponds to a line in $X$ if and only if $V_{k-1}$ is isotropic and $V_{k+1} \subset V_{k-1}^{\perp}$. It follows from this that $\overline{\mathcal{M}}_{0,1}(X, 1)$ can be identified with

$$
\overline{\mathcal{M}}_{0,1}(\mathrm{IG}, 1) \simeq\left\{\left(V_{k-1} \subset V_{k} \subset V_{k+1}\right): V_{k-1} \in \mathrm{IG}(k-1,2 n+1), V_{k+1} \subset V_{k-1}^{\perp}\right\} .
$$

Under this identification, ev $\mathrm{v}_{1}$ corresponds to the projection to the component $F_{k}$. Alternatively, one can use the embedding $X \subset \mathrm{IG}(k, 2 n+2)$, and use the identification

$\overline{\mathcal{M}}_{0,1}(\mathrm{IG}(k, 2 n+2), 1) \simeq\left\{\left(V_{k-1}^{\prime} \subset V_{k}^{\prime} \subset V_{k+1}^{\prime}\right): V_{k-1} \in \mathrm{IG}(k-1,2 n+2), V_{k+1}^{\prime} \subset\left(V_{k-1}^{\prime}\right)^{\perp}\right\}$

where the last variety is the flag bundle $\mathcal{F} \ell\left(1,2 ;\left(\mathcal{S}_{k-1}^{\prime}\right)^{\perp} / \mathcal{S}_{k-1}^{\prime}\right)$ over $\operatorname{IG}(k-1,2 n+2)$, and where $\mathcal{S}_{k-1}^{\prime}$ is the tautological subbundle. Then the moduli space of lines in $X$ is the incidence variety consisting the triples $\left(V_{k-1}^{\prime} \subset V_{k}^{\prime} \subset V_{k+1}^{\prime}\right)$ as above such that in addition $V_{k+1}^{\prime} \subset \mathbb{C}^{2 n+1}$. This variety can be realized as a "flag sheaf"

$$
\mathcal{F} \ell\left(1,2 ; \mathcal{S}_{k-1}^{\perp} / \mathcal{S}_{k-1}\right) \rightarrow \mathrm{IG}(k-1,2 n+1),
$$

where $\mathcal{S}_{k-1}$ is the tautological subbundle over the variety of kernels $\operatorname{IG}(k-1,2 n+1)$. This a not a flag bundle because $\mathcal{S}_{k-1}^{\perp}$ jumps rank when going from the open to the closed orbit in $\operatorname{IG}(k-1,2 n+1)$, but it is a flag bundle when restricted to either the open or the closed orbits.

\subsection{Lines intersecting the open orbit $X^{\circ}$}

In what follows we analyze the open subvariety $\mathcal{M}^{\circ} \subset \overline{\mathcal{M}}_{0,1}$ (IG, 1$)$ defined by

$$
\mathcal{M}^{\circ}:=\left\{(p, L): L \cap X^{\circ} \neq \emptyset\right\} .
$$

This parametrizes 1-pointed lines intersecting the open orbit in IG. Since the kernel of a line $L$ intersecting $X^{\circ}$ cannot contain $\mathbf{e}_{1}$, variety $\mathcal{M}^{\circ}$ can be realized as the flag bundle $\mathcal{F} \ell\left(1,2 ; \mathcal{S}_{k-1}^{\perp} / \mathcal{S}_{k-1}\right)$ over the open orbit IG $(k-1,2 n+1)^{\circ}$, where $\mathcal{S}_{k-1}$ denotes the tautological subbundle. In this case $\operatorname{rank}\left(\mathcal{S}_{k-1}^{\perp}\right)=2 n+1-(k-1)$.

Let $\pi: \mathcal{M}^{\circ} \rightarrow X$ denote the natural projection map. Key to the calculation of the GW invariants is the following result, analyzing the geometry of the fibres of $\pi$. 
Theorem 8.1.1. (a) The natural projection map $\pi: \mathcal{M}^{\circ} \rightarrow \mathrm{IG}(k, 2 n+1)$ is surjective, and all its fibers are irreducible, generically smooth, of dimension $\operatorname{dim} \mathcal{M}^{\circ}-$ $\operatorname{dim} \operatorname{IG}(k, 2 n+1)$.

(b) The inverse image $\pi^{-1}\left(X_{c}\right)$ is isomorphic to an $\mathrm{Sp}_{2 n+1}$ orbit in $\mathrm{IF}(k-1, k, k+$ $1 ; 2 n+1)$. In particular, it is smooth and irreducible.

Before proving the theorem, we recall the description of the $S p_{2 n+1}$-orbits of the odd-symplectic 3 -step partial flag $\operatorname{IF}(k-1, k, k+1 ; 2 n+1)$ :

$$
\begin{aligned}
& K_{1}=\left\{V_{k-1} \subset V_{k} \subset V_{k+1} \in \operatorname{IF}(k-1, k, k+1 ; 2 n+1): \mathbf{e}_{1} \in V_{k-1}\right\} \\
& K_{2}=\left\{V_{k-1} \subset V_{k} \subset V_{k+1} \in \operatorname{IF}(k-1, k, k+1 ; 2 n+1): \mathbf{e}_{1} \in V_{k}, \mathbf{e}_{1} \notin V_{k-1}\right\} \\
& K_{3}=\left\{V_{k-1} \subset V_{k} \subset V_{k+1} \in \operatorname{IF}(k-1, k, k+1 ; 2 n+1): \mathbf{e}_{1} \in V_{k+1}, \mathbf{e}_{1} \notin V_{k}\right\} \\
& K_{4}=\left\{V_{k-1} \subset V_{k} \subset V_{k+1} \in \operatorname{IF}(k-1, k, k+1 ; 2 n+1): \mathbf{e}_{1} \notin V_{k+1}\right\} .
\end{aligned}
$$

We also need the following lemma:

Lemma 8.1.2. Let $L$ be a line such that $L \cap X_{c} \neq \emptyset$ and $L \cap X^{\circ} \neq \emptyset$. Then Span $L$ is an isotropic subspace in $\mathbb{C}^{2 n+1}$.

Proof. Let $x \in L \cap X_{c}$ and $y \in L \cap X_{1}^{\circ}$. Since $x \in X_{c}$ and $y \notin X_{c}$ we can choose a basis $\left\{\mathbf{e}_{1}, x_{1}, \cdots, x_{k-1}\right\}$ for $x$ such that $\left\{x_{1}, \cdots, x_{k-1}\right\}$ is a basis for $x \cap y$ and choose a basis $\left\{x_{1}, \cdots, x_{k-1}, f\right\}$ for $y$. Then $\left\{\mathbf{e}_{1}, x_{1}, \cdots, x_{k-1}, f\right\}$ is a basis for Span $L=\langle x, y\rangle$. Clearly $\left\langle x_{i}, f\right\rangle=0$ and since $\mathbf{e}_{1} \in \operatorname{ker} \omega$ it follows that $\left\langle\mathbf{e}_{1}, f\right\rangle=0$. This finishes the proof.

We note that this is the best result possible. For instance let $n=k=2$ and consider the line that contains the $T$-fixed points $(1<3)$ and $(1<\overline{3})$ (this is a line included in the closed orbit $\left.X_{c} \simeq \operatorname{IG}(1,4) \simeq \mathbb{P}^{3}\right)$. Then $\operatorname{Span} L=\left\langle\mathbf{e}_{1}, \mathbf{e}_{3}, \mathbf{e}_{3}\right\rangle$ is not isotropic, because $\omega\left(\mathbf{e}_{3}, \mathbf{e}_{\overline{3}}\right)=1$. Similarly, the line joining $(2<3)$ to $(3<\overline{2})$ (a line in the open orbit) has again non-isotropic span; see Figure 5.1 below for more examples.

We will need to calculate $\operatorname{dim} K_{2}$. For that, observe that to construct a triple in $K_{2}$ one first chooses $V_{k} \in \mathrm{IG}(k, 2 n+1)_{c} \simeq \mathrm{IG}(k-1,2 n)$, then $V_{k-1}$ in an open set in $\operatorname{Gr}\left(k-1, V_{k}\right)$, and then finally an open set of $V_{k+1} \in \operatorname{Gr}\left(1, V_{k-1}^{\perp} / V_{k}\right.$ ). (The spaces $V_{k+1}$ obtained this way are automatically isotropic, because $\mathbf{e}_{1} \in V_{k}$.) This yields

$$
\begin{array}{r}
\operatorname{dim} K_{2}=\operatorname{dim} \operatorname{IG}(k-1,2 n)+(k-1)+(2 n-2 k+1) \\
=(k-1)(2 n-k+1)-\frac{(k-1)(k-2)}{2}+2 n-k .
\end{array}
$$

Proof of Theorem 8.1.1. The definition of $\mathcal{M}^{\circ}$ implies that $\pi$ is surjective over the open orbit $X^{\circ}$. By [9, Prop. 2.3] this is a locally trivial fibration, and because both $\mathcal{M}^{\circ}$ and $X^{\circ}$ are smooth and irreducible, it follows that the fibers over $X^{\circ}$ are also smooth and irreducible. Notice that the same result implies that $\pi^{-1}\left(X_{c}\right)$ is a locally trivial fibration over $X_{c}$. To prove (a) it remains to show that the fibre $\pi^{-1}(1 . P)$ is nonempty, irreducible and generically smooth. 
As explained in $\S 5.1$, there is a line joining 1.P to $\left\langle e_{2}, e_{3}, \ldots, e_{k}, e_{k+1}\right\rangle \in X^{\circ}$. Thus $\pi^{-1}(1 . P) \neq \emptyset$. We prove next that the reduced support $\left(\pi^{-1}\left(X_{c}\right)\right)_{r e d}$ is irreducible. This implies that $\pi^{-1}\left(X_{c}\right)$ is again irreducible. Then we will use a local calculation to find an open dense set of $\pi^{-1}(1 . P)$ where it is smooth. That will simultaneously prove both (a) and (b).

To start, there is a bijective morphism $K_{2} \rightarrow\left(\pi^{-1}\left(X^{c}\right)\right)_{\text {red }}$ defined as follows: to each pointed line $p \in L \subset \mathrm{IG}$ such that $p \in L \cap X_{c}$ and $L \cap X^{\circ} \neq \emptyset$ one associates the element ( $\operatorname{ker} L, p, \operatorname{Span} L) \in \operatorname{IF}(k-1, k, k+1 ; 2 n+1)$. (The fact that the span of $L$ is isotropic follows from Lemma 8.1.2.) Conversely, to each element $\left(V_{k-1} \subset V_{k} \subset V_{k+1}\right) \in \operatorname{IF}(k-1, k, k+1 ; 2 n+1)$ such that $V_{k} \in X_{c}$ (i.e. $\left.\mathbf{e}_{1} \in V_{k}\right)$ and $\mathbf{e}_{1} \notin V_{k-1}$ one associates a line $L:=\mathbb{P}\left(V_{k+1} / V_{k-1}\right)$ and the point $V_{k} \in X_{c}$. Since $V_{k+1}$ is isotropic it follows that $L$ is a line in IG; the condition $\mathbf{e}_{1} \notin F_{k-1}$ implies that $L$ cannot be included in the closed orbit, so $L \cap X^{\circ} \neq \emptyset$. The fact that this is an algebraic morphism follows e.g. because $K_{2}$ is an orbit of $\mathrm{Sp}_{2 n+1}$. This proves that $\pi^{-1}\left(X^{c}\right)$ is irreducible. Since $\pi^{-1}\left(X_{c}\right) \rightarrow X_{c}$ is a locally trivial fibration, it follows that $\pi^{-1}(1 . P)$ is irreducible, and that it has dimension

$$
\operatorname{dim} \pi^{-1}(1 . P)=\operatorname{dim} K_{2}-\operatorname{dim} X_{c}=2 n-k=\operatorname{dim} \mathcal{M}^{\circ}-\operatorname{dim} X .
$$

Turning to smoothness, we will show that there exist open sets $U_{1} \subset X$ and $U_{2} \subset \mathcal{M}^{\circ}$ such that $1 . P \in U_{1}, U_{2} \subset \pi^{-1}(U), U_{i}$ 's are isomorphic to open sets in some affine spaces $\mathbb{A}^{N_{i}}, i=1,2$ (for appropriate $N_{i}$ ), and such that the induced map $U_{2} \rightarrow U_{1}$ is smooth. Using the coordinate charts in $\operatorname{Gr}(k, 2 n+1)$ one defines the open set $U_{1}$ around $1 . P$ to be given by

$$
\left(\begin{array}{cccccc}
1 & 0 & 0 & \cdots & 0 & 0 \\
0 & 1 & 0 & \cdots & 0 & 0 \\
0 & 0 & 1 & \cdots & 0 & 0 \\
\vdots & \vdots & \vdots & \cdots & \vdots & \vdots \\
0 & 0 & 0 & \cdots & 0 & 1 \\
a_{k+1,1} & a_{k+1,2} & a_{k+1,3} & \cdots & a_{k+1, k-1} & a_{k+1, k} \\
\vdots & \vdots & \vdots & \cdots & \vdots & \vdots \\
a_{\overline{k+1}, 1} & a_{\overline{k+1}, 2} & a_{\overline{k+1}, 3} & \cdots & a_{\overline{k+1}, k-1} & a_{\overline{k+1}, k}^{+\bullet} \\
a_{\bar{k}, 1}^{\bullet} & a_{\bar{k}, 2}^{\bullet} & a_{\bar{k}, 3}^{\bullet} & \cdots & a_{\bar{k}, k-1}^{\bullet} & a_{\bar{k}, k} \\
\vdots & \vdots & \vdots & \cdots & \vdots & \vdots \\
a_{\overline{4}, 1}^{\bullet} & a_{\overline{4}, 2}^{\bullet} & a_{\overline{4}, 3}^{\bullet} & \cdots & a_{\overline{4}, k-1} & a_{\overline{4}, k} \\
a_{\overline{3}, 1}^{\bullet} & a_{\overline{3}, 2}^{\bullet} & a_{\overline{3}, 3} & \cdots & a_{\overline{3}, k-1} & a_{\overline{3}, k} \\
a_{\overline{2}, 1}^{\bullet} & a_{\overline{2}, 2} & a_{\overline{2}, 3} & \cdots & a_{\overline{2}, k-1} & a_{\overline{2}, k}
\end{array}\right) .
$$

A space $V \in U_{1}$ is given by the $k$ vector columns $v_{1}, \ldots, v_{k}$. Each of the coordinates $a_{i, j}^{\bullet}$ is determined from the others by imposing the condition that $V$ is isotropic. It turns out that these conditions give linear equations in each of the $a_{i, j}^{\bullet}$, therefore $U$ is isomorphic to an affine space $\mathbb{A}^{\operatorname{dim} X}$. 
To define $U_{2}$, we first observe that an open set in the (dual) projective space of codimension 1 subspaces $V_{k-1} \subset V=\left\langle v_{1}, \ldots, v_{k}\right\rangle \in U_{1}$ where $\mathbf{e}_{1} \notin V_{k-1}$ is given by $\left\langle v_{i}+c_{i} v_{1}: 2 \leq i \leq k, c_{i} \in \mathbb{C}\right\rangle$. Then an open set $U_{2}$ around triples containing such $V_{k}$ is given by the column span of the matrix

$$
\left(\begin{array}{ccccc|c|c}
c_{2} & c_{3} & \cdots & c_{k-1} & c_{k} & 1 & 0 \\
1 & 0 & \cdots & 0 & 0 & 0 & 0 \\
0 & 1 & \cdots & 0 & 0 & 0 & 0 \\
\vdots & \vdots & \cdots & \vdots & \vdots & \vdots & \vdots \\
0 & 0 & \cdots & 0 & 1 & 0 & 0 \\
a_{k+1,2}+c_{2} a_{k+1,1} & a_{k+1,3}+c_{3} a_{k+1,1} & \cdots & a_{k+1, k-1}+c_{k-1} a_{k+1,1} & a_{k+1, k}+c_{k} a_{k+1,1} & a_{k+1,1} & 1 \\
\vdots & \vdots & \cdots & \vdots & \vdots & \vdots & \vdots \\
a_{\overline{k+1,2}}+c_{2} a_{\overline{k+1}, 1} & a_{\overline{k+1}, 3}+c_{3} a_{\overline{k+1}, 1} & \cdots & a_{\overline{k+1}, k-1}+c_{k-1} a_{\overline{k+1}, 1} & a_{\overline{k+1}, k}+c_{k} a_{\overline{k+1}, 1} & a_{\overline{k+1}, 1} & d_{\overline{k+1}} \\
a_{\bar{k}, 2}^{\bullet}+c_{2} a_{\bar{k}, 1}^{\bullet} & a_{\bar{k}, 3}^{\bullet}+c_{3} a_{\bar{k}, 1}^{\bullet} & \cdots & a_{\bar{k}, k-1}^{\bullet}+c_{k-1} a_{\bar{k}, 1}^{\bullet} & a_{\bar{k}, k}+c_{k} a_{\bar{k}, 1}^{\bullet} & a_{\bar{k}, 1}^{\bullet} & d_{\bar{k}}^{\bullet} \\
\vdots & \vdots & \cdots & \vdots & \vdots & \vdots & \vdots \\
a_{\overline{4}, 2}^{\bullet}+c_{2} a_{\overline{4}, 1}^{\bullet} & a_{\overline{4}, 3}^{\bullet}+c_{3} a_{\overline{4}, 1}^{\bullet} & \cdots & a_{\overline{4}, k-1}+c_{k-1} a_{\overline{4}, 1}^{\bullet} & a_{\overline{4}, k}+c_{k}^{\bullet} a_{\overline{4}, 1} & a_{\overline{4}, 1}^{\bullet} & d_{\overline{4}}^{\bullet} \\
a_{\overline{3}, 2}^{\bullet}+c_{2} a_{\overline{3}, 1}^{\bullet} & a_{\overline{3}, 3}+c_{3} a_{\overline{3}, 1}^{\bullet} & \cdots & a_{\overline{3}, k-1}+c_{k-1} a_{\overline{3}, 1}^{\bullet} & a_{\overline{3}, k}+c_{k} a_{\overline{3}, 1}^{\bullet} & a_{\overline{3}, 1}^{\bullet} & d_{\overline{3}}^{\bullet} \\
a_{\overline{2}, 2}+c_{2} a_{\overline{2}, 1}^{\bullet} & a_{\overline{2}, 3}+c_{3} a_{\overline{2}, 1}^{\bullet} & \cdots & a_{\overline{2}, k-1}+c_{k-1} a_{\overline{2}, 1}^{\bullet} & a_{\overline{2}, k}+c_{k} a_{\overline{2}, 1}^{\bullet} & a_{\overline{2}, 1}^{\bullet} & d_{\overline{2}}^{\bullet}
\end{array}\right) .
$$

Again the coordinates $d_{i, j}^{\bullet}$ are determined from linear equations, thus $U_{2}$ is isomorphic to an open set $\mathbb{A}^{\operatorname{dim} \mathcal{M}^{\circ}}$. In these coordinates the map $\pi_{U_{2}}: U_{2} \rightarrow U_{1}$ becomes a linear map given by $c_{i} \mapsto 0$ and $d_{i} \mapsto 0$. In particular, this map is smooth, and the fiber $\pi^{-1}(1 . P) \cap U_{2}$ is smooth. This finishes the proof.

\subsection{Lines in the closed orbit}

As before let $X:=\operatorname{IG}(k, 2 n+1)$ and let $\overline{\mathcal{M}}:=\overline{\mathcal{M}}_{0,1}(X, 1)$. Consider the closed subvariety

$$
\mathcal{M}_{c}:=\overline{\mathcal{M}} \backslash \mathcal{M}^{\circ}=\left\{(p, L) \in \overline{\mathcal{M}}_{0,1}(X, 1): L \subset X_{c}\right\},
$$

which consists of lines included in the closed orbit. In terms of triples of flags this consists of triples $\left(V_{k-1} \subset V_{k} \subset V_{k+1}\right)$ such that $V_{k-1}$ belongs to the closed orbit in $\operatorname{IG}(k-1,2 n+1)$ (i.e $\mathbf{e}_{1} \in V_{k-1}$ ), and $V_{k+1} \subset V_{k-1}^{\perp}$. Because the symplectic form in $\mathbb{C}^{2 n+1}$ is general, this is a smooth subvariety of $\operatorname{IF}(k-1, k, k+1 ; 2 n+1)$, and the universal property for the moduli space of stable maps gives a bijective morphism to $\overline{\mathcal{M}}_{0,1}\left(X_{c}, 1\right)$. It follows that $\mathcal{M}_{c}$ is isomorphic to the moduli space $\overline{\mathcal{M}}_{0,1}\left(X_{c}, 1\right)$. Recall that $X_{c}$ is isomorphic to the homogeneous space $\operatorname{IG}(k-1,2 n)$, thus the moduli space $\overline{\mathcal{M}}_{0,1}\left(X_{c}, 1\right)$ is a smooth, irreducible variety of dimension

$$
\operatorname{dim} \mathcal{M}_{c}=\operatorname{dim} \operatorname{IG}(k-1,2 n)+2 n+1-(k-1)-2=\operatorname{dim} \mathrm{IG}(k-1,2 n)+2 n-k .
$$

(Note the coincidence $\operatorname{dim} \mathcal{M}_{c}=\operatorname{dim} K_{2}$. We recall the following result, proved in Thm. 2.5 and Cor. 3.3 from [9]: 
Lemma 8.2.1. For every $V \in X_{c}$, the fibre $\mathrm{ev}_{1}^{-1}(V)$ of the restricted map $\mathrm{ev}_{1}$ : $\overline{\mathcal{M}}_{0,1}\left(X_{c}, 1\right) \rightarrow X_{c}$ is an irreducible, normal variety of dimension $\operatorname{dim} \mathcal{M}_{c}-\operatorname{dim} X_{c}$.

We combine the previous lemma to Theorem 8.1.1 to obtain the main result of this section.

Theorem 8.2.2. Consider the evaluation map $\mathrm{ev}_{1}: \overline{\mathcal{M}}_{0,1}(X, 1) \rightarrow X$. Then the following hold:

(a) For any $V \in \operatorname{IG}(k, 2 n+1)$, the fibre $\mathrm{ev}_{1}^{-1}(V)$ is pure dimensional of dimension $\operatorname{dim} \overline{\mathcal{M}}_{0,1}(\mathrm{IG}(k, 2 n+1), 1)-\operatorname{dim} \mathrm{IG}(k, 2 n+1)$, and each of its components is generically smooth. In particular, $\mathrm{ev}_{1}$ is flat.

(b) For any Schubert variety $X(w) \subset X_{c}$, the preimage $\mathrm{ev}_{1}^{-1}(X(w))$ has two irreducible components:

$$
\mathrm{ev}_{1}^{-1}(X(w)):=A_{1} \cup A_{2},
$$

where $A_{1}$ is the closure of the subvariety of pointed lines $(p, L)$ such that $L \cap X^{\circ} \neq \emptyset$, and $A_{2}$ is the closed subscheme corresponding to $(p, L)$ such that $L$ is included in the closed orbit $X_{c}$. Further, each irreducible component is generically smooth of expected dimension $\operatorname{dim} \overline{\mathcal{M}}_{0,1}(X, 1)-\operatorname{codim}_{X} X(w)$.

Proof. Since $\mathrm{Sp}_{2 n+1}$ acts transitively on the open orbit $X^{\circ}$, the morphism $\mathrm{ev}_{1}$ is flat, and the fibres have the stated dimension. Transitivity implies that all fibers over the closed orbit are isomorphic, thus it suffices to take $V=1 . P$. Let $F:=\mathrm{ev}_{1}^{-1}(1 . P)$ be the fibre. Recall the notation $\mathcal{M}^{\circ}$ and $\mathcal{M}_{c}$. Clearly $F$ can be written as the disjoint union $F=F^{\circ} \cup F_{c}$ where $F^{\circ}:=F \cap \mathcal{M}^{\circ}$ is open in $F$ and $F_{c}:=F \backslash F^{\circ}$ is closed in $\mathcal{M}_{c}$. It follows from Theorem 8.1.1 that $F^{\circ}$ is irreducible, generically reduced, and of the stated dimension. On the other side, Lemma 8.2.1 implies that $F_{c}$ is irreducible, generically reduced, of dimension

$$
\operatorname{dim} F_{c}=\operatorname{dim} \mathcal{M}_{c}-\operatorname{dim} X_{c}=\operatorname{dim} \mathcal{M}-\operatorname{dim} X
$$

(the last equality is a simple calculation). Therefore $F_{c}$ cannot in the closure of $F^{\circ}$, and the statements about $F$ hold. ${ }^{1}$ The flatness follows from [45, Theorem 23.1], taking into account that both source and target of $\mathrm{ev}_{1}$ are smooth varieties, and that all fibers have the same dimension. Flatness implies that the GW variety $G W_{1}(w)$ from part (b) is pure dimensional of expected dimension. Further, using transitivity and applying [9, Prop. 2.3] to each irreducible component of $\mathrm{ev}_{1}^{-1}\left(X_{c}\right)$ implies that the map ev $\mathrm{ev}_{1} \mathrm{ev}_{1}^{-1}\left(X_{c}\right) \rightarrow X_{c}$ is a locally trivial fibration with fibre $F$. Then the restriction to $\mathrm{ev}_{1}^{-1}(X(w))$ is a locally trivial fibration over $X(w)$ with fibre $F$, and the statement in (b) follows.

\footnotetext{
${ }^{1} \mathrm{~A}$ simpler way to see that $F_{c} \subsetneq \overline{F^{\circ}}$ is to notice that every line in $F^{\circ}$ has isotropic span, therefore any line in the closure must satisfy the same property. But we have seen that there exist lines in $X_{c}$ with non isotropic span.
} 


\subsection{Lines with two marked points}

Define $\xi: \overline{\mathcal{M}}_{0,2}(X, 1) \longrightarrow \overline{\mathcal{M}}_{0,1}(X, 1)$ to be the map the map forgetting the second marked point.

Proposition 8.3.1. The forgetful map $\xi: \overline{\mathcal{M}}_{0,2}(X, 1) \longrightarrow \overline{\mathcal{M}}_{0,1}(X, 1)$ is a locally trivial $\mathbb{P}^{1}$-fibration.

Proof. Consider the embedding $X \subset \mathrm{Gr}:=\mathrm{Gr}(k, 2 n+1)$. We first prove the statement with $X$ replaced by Gr. Recall that the moduli space $\overline{\mathcal{M}}_{0,1}(\mathrm{Gr}, 1)$ may be identified to the partial flag manifold $\mathrm{Fl}(k-1, k, k+1 ; 2 n+1)$. It follows in particular that $\overline{\mathcal{M}}_{0,1}(\mathrm{Gr}, 1)$ admits a transitive action of $\mathrm{SL}:=\mathrm{SL}_{2 n+1}$. Then by [9, Prop. 2.3] the forgetful map $\overline{\mathcal{M}}_{0,2}(\mathrm{Gr}, 1) \rightarrow \overline{\mathcal{M}}_{0,1}(\mathrm{Gr}, 1)$ is an SL-equivariant locally trivial fibration with fibres isomorphic to $\mathbb{P}^{1}$. Consider the commutative diagram:

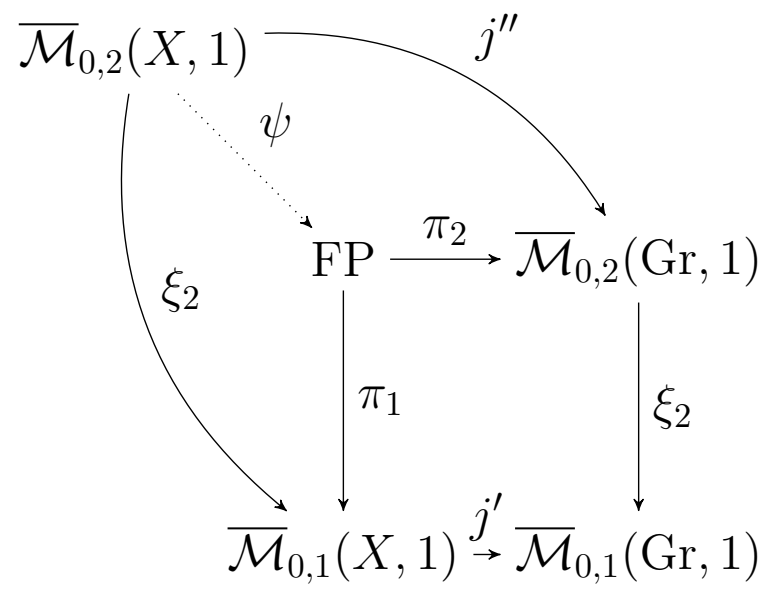

where FP denotes the fibre product and $j^{\prime}, j$ " are the closed embeddings determined by the embedding $X \subset$ Gr. The map $\psi$ is determined by the universal property for fibre products. It is easy to check that $\psi$ is bijective. Since both $\overline{\mathcal{M}}_{0,2}(X, 1)$ and $F P$ are smooth varieties it is in fact an isomorphism, by Zariski's Main Theorem (cf. $[31,53]$ ). Since the right vertical arrow is a $\mathbb{P}^{1}$-fibration, so is the left vertical arrow $F P \simeq \overline{\mathcal{M}}_{0,2}(X, 1) \rightarrow \overline{\mathcal{M}}_{0,1}(X, 1)$. This proves the statement.

Combining Proposition 8.3.1 and Theorem 8.2.2 imply the main result of this section. Recall that $G W_{1}(w)$ denotes the Gromov-Witten variety $\mathrm{ev}_{1}^{-1}(X(w))$. Obviously $\mathrm{ev}_{1}$ is the composition of the forgetful map $\xi$ with the evaluation map from $\overline{\mathcal{M}}_{0,1}(X, 1)$.

Corollary 8.3.2. Consider the Schubert variety $X(w) \subset X_{c}$. Then the GromovWitten variety $G W_{1}(w)$ has two irreducible components

$$
G W_{1}(w)=G W_{1}^{(1)}(w) \cup G W_{1}^{(2)}(w),
$$

where $G W_{1}^{(1)}(w)=\xi^{-1}\left(A_{1}\right)$ is the closure of the subvariety corresponding to lines $L$ such that $L \cap X^{\circ} \neq \emptyset$, and $G W_{1}^{(2)}(w)=\xi^{-1}\left(A_{2}\right)$ is the closed subscheme corresponding 
to lines $L$ included in the closed orbit $X_{c}$. Further, each irreducible component is generically smooth and it has dimension $\operatorname{dim} \overline{\mathcal{M}}_{0,2}(X, 1)-\operatorname{codim}_{X} X(w)$. 


\section{Chapter 9}

\section{Line neighborhoods}

We use the notation from the previous section. Recall that $W^{2 n+1}$ denotes the subset of minimal length representatives for the odd-symplectic Grassmannian $X$, regarded as a Schubert variety in $\operatorname{IG}(k, 2 n+2)$. In this section we analyze the curve neighborhoods $\Gamma_{1}(X(w))$ (i.e. the line neighborhood) in the case when $X(w) \subset X_{c}$. By Theorem 7.0.1 these are the only ones which may contribute to non-zero GW invariants. Recall from Corollary 8.3.2 that the Gromov-Witten variety $G W_{1}(w)$ has precisely two components of expected dimension. It follows that the curve neighborhood $\Gamma_{1}(w)$ has at most two components, and we have an equality

$$
\Gamma_{1}(w)=\Gamma_{1}^{(1)}(w) \cup \Gamma_{1}^{(2)}(w),
$$

where $\mathrm{ev}_{2}: G W_{1}^{(i)}(w) \rightarrow \Gamma_{1}^{(i)}(w):=\operatorname{ev}_{2}\left(G W_{1}^{(i)}(w)\right)(i=1,2)$. By definition, $\Gamma_{1}^{(1)}(w) \cap$ $X^{\circ} \neq \emptyset, \Gamma_{1}^{(2)}(w) \subset X_{c}$, and each of $\Gamma_{1}^{(i)}(w)$ is irreducible and stable under the standard Borel subgroup; therefore it must be a Schubert variety. Further, since the second component $G W_{1}^{(2)}(w)$ is the GW variety of lines in the closed orbit $X_{c}$ - isomorphic to the homogeneous space $\operatorname{IG}(k-1,2 n)$ - it follows from [16] that

$$
\Gamma_{1}^{(2)}(w)=X\left(w \cdot O_{2}\right)
$$

where $X\left(O_{2}\right)=\Gamma_{1}^{X_{c}}(i d)$ (the curve neighborhood of the Schubert point in $X_{c}$ ). We will identify $\mathrm{O}_{2}$ explicitly below, together with the first component $\Gamma_{1}^{(1)}(w)$, and we will calculate the dimension of each component. Before starting, we remark that the behavior of curve neighborhoods for the quasi-homogeneous space $X$ is already different from that of any homogeneous space. For the latter, it was proved in [9] and [16] that any curve neighborhood of a Schubert variety is a single Schubert variety. The next result shows that this already fails for $\Gamma_{1}(i d)$.

Proposition 9.0.1. Let $O_{1}=(2<3<\cdots<k<\overline{k+1})$ and $O_{2}=(1<3<4<$ $\cdots<k<\overline{2})$. Then the line neighborhood of the Schubert point in IG is $\Gamma_{1}(i d)=$ $X\left(O_{1}\right) \cup X\left(O_{2}\right)$ and $\ell\left(O_{1}\right)=\ell\left(O_{2}\right)=2 n+1-k$. (Observe that this equals $\operatorname{deg} q-1$.)

Proof. Observe that $O_{1}$ and $O_{2}$ are indeed joined by a line to the identity, as shown in section 5.1 above. The fact that $O_{1}$ and $O_{2}$ have the length $2 n+1-k$ follows 
immediately from the equation (11.1) below, where we describe $O_{1}, O_{2}$ in terms of strict partitions.

Recall that $W$ denotes the Weyl group of the symplectic group $\mathrm{Sp}_{2 n+2}$.

Theorem 9.0.2. Let $w=\left(w_{1}<\cdots<w_{k}\right) \in W^{2 n+1}$ such that $X\left(w W_{P}\right) \subset X_{c}$. Then the cosets $w \cdot o_{1} W_{P}$ and $w \cdot O_{1} W_{P}$ have representatives in $W^{2 n+1}$ and

$$
\Gamma_{1}\left(X\left(w W_{P}\right)\right)=X\left(w \cdot O_{1} W_{P}\right) \cup X\left(w \cdot o_{1} W_{P}\right) .
$$

Proof. The existence of representatives in $W^{2 n+1}$ follows from Lemma 2.4.2. Next, since both varieties are $B$-stable, it suffices to check they have the same $T$-fixed points. Using the action of $\mathrm{Sp}_{2 n+1}$, a line passing through the Schubert point $X(i d)$ can be translated so it contains any point in the closed orbit. In particular, a $T$-stable line guaranteed by Proposition 9.0.1, joining $X(i d)$ to $O_{1} W_{P}$ (respectively $o_{1} W_{P}$ ) is translated to one joining any $T$-fixed point $v W_{P} \in X\left(w W_{P}\right)$ to $v O_{1} W_{P}$ (respectively $\left.v o_{1} W_{P}\right)$. Since the minimal length representatives satisfy $v \leq w$ it follows that $v O_{1} \leq v \cdot O_{1} \leq w \cdot O_{1}$, and similarly for $O_{2}$. Then the same inequalities hold for the cosets. We deduce that $\Gamma_{1}\left(X\left(w W_{P}\right)\right) \subset X\left(w \cdot O_{1} W_{P}\right) \cup X\left(w \cdot o_{1} W_{P}\right)$. For the converse inclusion we will consider only lines $L$ which intersect both $X\left(w W_{P}\right)$ and the open orbit $X^{\circ}$ (those included in the closed orbit are already accounted from equality $(9.1))$. Let $v=\left(w \cdot O_{1}\right) O_{1}^{-1}$, where the products are performed in $W$. Then $v \leq w$ by [16, Prop.3.1] and $v O_{1}=w \cdot O_{1}$ in $W$. If $L$ is the line joining $X(i d)$ to $O_{1} W_{P}$ in $X$ then $v . L$ joins $v W_{P} \in X\left(w W_{P}\right)$ to $v O_{1} W_{P}=\left(w \cdot O_{1}\right) W_{P} \in X\left(w \cdot O_{1} W_{P}\right)$. This proves the required inclusion.

We record an immediate consequence of Lemma 2.3.6, which gives necessary and sufficient conditions for the components of the curve neighborhood to have the expected dimension.

Lemma 9.0.3. Let $w \in W^{2 n+1}$ such that $X\left(w W_{P}\right) \subset X_{c}$, and let $z \in\left\{O_{1}, O_{2}\right\}$. Then $\operatorname{dim} X\left(w \cdot z W_{P}\right)-\operatorname{dim} X\left(w W_{P}\right) \leq \operatorname{deg} q-1$. Further, the following are equivalent:

(i) $\operatorname{dim} X\left(w \cdot z W_{P}\right)-\operatorname{dim} X\left(w W_{P}\right)=\operatorname{deg} q-1$;

(ii) $\ell(w \cdot z)=\ell(w)+\ell(z), w \cdot z=w z$ and $w \cdot z$ is a minimal length representative in $W^{2 n+1}$. 
Figure 9.1: The figure illustrates $\Gamma_{1}(X(w))$ on the moment graph. The colored edges leaving each red vertex is either connecting to $w \cdot O_{1}$ or $w \cdot O_{2}$.

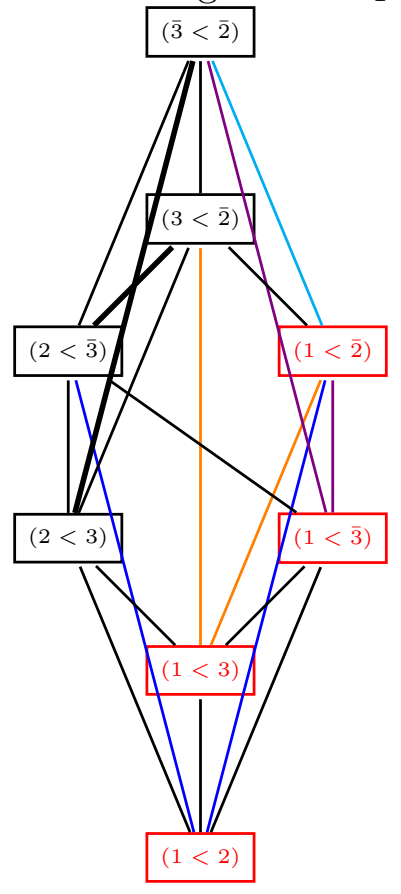




\section{Chapter 10}

\section{Gromov-Witten invariants of lines}

The main result of this section is the following:

Theorem 10.0.1. Let $X(w) \subset X_{c}$ be a Schubert variety in the closed orbit of $X$, let $z \in\left\{O_{1}, O_{2}\right\}$. Consider the restricted evaluation map

$$
\mathrm{ev}_{2}: G W_{1}^{(i)}(w) \rightarrow X\left(w \cdot O_{i}\right) \quad(i=1,2) .
$$

Then $\operatorname{dim} G W_{1}^{(i)}(w) \geq \operatorname{dim} X\left(w \cdot O_{i} W_{P}\right)$ with equality if and only if the restricted map $\mathrm{ev}_{2}$ is birational. In particular, the following holds:

$$
\left(\mathrm{ev}_{2}\right)_{*}\left[G W_{1}^{(i)}(w)\right]= \begin{cases}{\left[X\left(w \cdot O_{i}\right)\right]} & \text { if } w \cdot O_{i} \in W^{2 n+1} \text { and } \ell\left(w \cdot O_{i}\right)=\ell(w)+\ell\left(O_{i}\right) \\ 0 & \text { otherwise }\end{cases}
$$

Proof. By definition $X\left(w \cdot O_{i} W_{P}\right)=\mathrm{ev}_{2}\left(G W_{1}^{(i)}(w)\right)$, therefore the inequality on dimensions is immediate. In the case of equality it remains to prove the birationality statement. First observe that in this case $\operatorname{dim} X\left(w \cdot O_{i} W_{P}\right)=\operatorname{dim} X\left(w W_{P}\right)+\operatorname{deg} q-1$, and by Lemma 9.0.3 $w \cdot O_{i}$ is a minimal length representative satisfying $\ell\left(w \cdot O_{i}\right)=$ $\ell(w)+\ell\left(O_{i}\right)$. Given this, we will drop $W_{P}$ from the notation.

Recall from Corollary 8.3.2 that $G W_{1}^{(i)}(w)$ is irreducible and generically smooth. Since the evaluation map $\mathrm{ev}_{2}$ is $B$-equivariant, [9, Prop. 2.3] implies that $\mathrm{ev}_{2}$ is a locally trivial fibration over the open cell $X\left(w \cdot O_{i} W_{P}\right)^{\circ}$. The preimage $\operatorname{ev}_{2}^{-1}\left(X\left(w \cdot O_{i}\right)\right)$, being open and dense, intersects the smooth locus of $G W_{1}^{(i)}(w)$. Therefore all fibres over the open cell are reduced, and to prove birationality it suffices to show that for some $x \in X\left(w \cdot O_{i}\right)^{\circ}$ there exists a unique line $L$ such that $x \in L$ and $L \cap X(w) \neq \emptyset$. If $i=2$ (i.e. the GW variety parametrizes lines included in the closed orbit of $\mathrm{X}$ ) this statement follows from [9, Prop.3.2]. We assume from now on that $i=1$.

We consider the fibre over $x=w O_{1}=w \cdot O_{1}$. The $w$-translate of the $T$-stable line joining $X(i d)$ and $O_{1}$ gives a $T$-stable line $L_{w}$ joining $w$ and $w O_{1}$. If $X(v) \subset X(w)$ such that $v \neq w$ then

$\operatorname{dim} \Gamma_{1}(X(w))-\operatorname{dim} X(v)=\ell\left(w \cdot O_{1}\right)-\ell(v)=2 n+1-k+(\ell(w)-\ell(v))>2 n+1-k$. 
Then theorems 6.2.3 and 9.0.2 imply that there is no line joining $X(v)$ to the open cell $X\left(w O_{1}\right)^{\circ}$. We deduce that any line passing through $w O_{1}$ and $X(w)$ cannot intersect the boundary $X(w) \backslash X(w)^{\circ}$ of $X(w)$. In particular, this proves that $L_{w}$ is the unique $T$-stable line in the fibre over $w O_{1}$. Let $L$ be any line such that $w O_{1} \in L$ and $y \in L \cap X(w)^{\circ}$. If $y=w$ then $L=L_{w}$ is $T$-stable, so assume $y \neq w$; in particular $L$ is not $T$-stable. We show that existence of this $L$ leads to a contradiction. Consider a general $\mathbb{C}^{*} \subset T$ such that the $T$ and $\mathbb{C}^{*}$ fixed points in $X$ coincide. (This $\mathbb{C}^{*}$ can be found by [6, Lemma 2.3], using that the $T$-action at the Schubert point $X(i d) \subset X$ is effective.) A line $t . L$ in the (infinite) family of lines $\left\{t . L: t \in \mathbb{C}^{*}\right\}$ contains $w \cdot O_{1}$ and it passes through t.y $\in X(w)^{\circ}$. The limits at 0 and $\infty$ exist by the properness of the appropriate Hilbert scheme [31, Proposition 3.9.8], and they correspond to two lines passing through two (distinct) $T$-fixed points $\lim _{t \rightarrow 0} t . y, \lim _{t \rightarrow \infty} t . y \in X(w)$. The two lines are necessarily $T$-stable, and this contradicts the uniqueness of $L_{w}$.

As a corollary, we can calculate the GW invariants for lines intersecting the closed orbit. (Those corresponding to lines intersecting the open orbit are equal to 0 , by Theorem 7.0.1.) Recall that $X(D i v)$ denotes the Schubert divisor in $X$.

Corollary 10.0.2. Let $u, w \in W^{2 n+1}$ such that $X(w) \subset X_{c}$. Then the GromovWitten invariant $\left\langle[X(\text { Div })]_{T},[X(w)]_{T},[X(u)]_{T}^{\vee}\right\rangle_{1}=1$ if $u=w O_{i}$ and $\ell(u)=\ell(w)+$ $\ell\left(O_{i}\right)$, and it is equal to 0 otherwise.

Proof. As in the proof of Theorem 7.0.1 we obtain

$$
\left\langle[X(\text { Div })]_{T},[X(w)]_{T},[X(u)]_{T}^{\vee}\right\rangle_{1}=\int_{\mathrm{IG}(k, 2 n+1)}^{T}[X(u)]_{T}^{\vee} \cap\left(\mathrm{ev}_{2}\right)_{*}\left(\mathrm{ev}_{1}^{*}[X(w)]_{T}\right) .
$$

(We omitted the virtual class, since for $d=1$ this is the actual fundamental class.) By Theorem 8.2.2 and Proposition 8.3.1, the evaluation map $\mathrm{ev}_{2}$ is flat. Then by Corollary 8.3.2,

$$
\mathrm{ev}_{1}^{*}[X(w)]_{T}=\left[e v_{1}^{-1}(X(w))\right]_{T}=\left[G W_{1}^{(1)}(w)\right]_{T}+\left[G W_{1}^{(2)}(w)\right]_{T} .
$$

Then the result follows from Theorem 10.0.1 and Poincaré duality.

The previous Corollary together with Theorem 7.0.1 give the quantum terms in the equivariant quantum Chevalley formula for $X$. Recall that the Chevalley formula is given by

$$
[X(D i v)]_{T} \star[X(w)]_{T}=\sum_{d \geq 0 ; u \in W^{2 n+1}} c_{D i v, w}^{u, d} q^{d}[X(u)]_{T}
$$

where $c_{\text {Div,w }}^{u, d}$ is a homogeneous polynomial of degree codim $X(w)+1-(\operatorname{codim} X(u)+$ $d \operatorname{deg} q$ ). The terms when $d=0$ (i.e. the non-quantum, equivariant coefficients) were obtained by Mihai in $[46,47]$. Those for $d>0$ are listed below. We remark that these coefficients were also calculated by Pech for $\mathrm{QH}^{*}(\mathrm{IG}(2,2 n+1))$ in $[55,56]$ and they were conjectured in few cases for $\mathrm{QH}^{*}(\mathrm{IG}(3,2 n+1))$. 
Theorem 10.0.3. Let $u, w \in W^{2 n+1}$ and $d>0$. The equivariant quantum Chevalley coefficients $c_{\text {Div, } w}^{u, d}=0$ for $d \geq 2$ or if $w(1) \neq 1$ (i.e. $X(w) \nsubseteq X_{c}$ ). If $d=1$ and $w(1)=1$ then

$$
c_{\text {Div,w }}^{u, d}= \begin{cases}1 & \text { if } u=w O_{i} \text { and } \ell(u)=\ell(w)+\ell\left(O_{i}\right) \\ 0 & \text { otherwise }\end{cases}
$$

In the next section we will re-write this formula in terms of partitions. 


\section{Chapter 11}

\section{Equivariant quantum Chevalley rule with $(n-k)$-strict partitions}

The goal of this section is to give an explicit formulation of the equivariant quantum Chevalley formula using partitions.

\subsection{A dictionary permutations - partitions}

In this section we introduce a variant of Buch, Kresch and Tamvakis $k$-strict partitions [13]. This variant, due to Pech [56], is convenient to describe the cohomology of the odd-symplectic Grassmannian $X=\mathrm{IG}(k, 2 n+1)$. Recall that if $P_{k}$ is the maximal parabolic subgroup of $\mathrm{Sp}_{2 n+2}$ determined by the simple root $\alpha_{k}$, then the minimal length representatives $W^{P_{k}}$ have the form $(w(1)<w(2)<\cdots<w(k))$. Consider the set of partitions $\lambda=\left(2 n+2-k \geq \lambda_{1} \geq \cdots \geq \lambda_{k} \geq 0\right)$ which are $(n+1-k)$-strict, i.e. $\lambda_{j}>\lambda_{j+1}$ whenever $\lambda_{j}>n+1-k$. We denote this set by $\Lambda_{k}^{2 n+2}$. There is a bijection between $\Lambda_{k}^{2 n+2}$ and the set $W^{P_{k}}$ of minimal length representatives given by:

$\lambda \mapsto w$ is defined by $w(j)=2 n+3-k-\lambda_{j}+$ $\#\left\{i<j: \lambda_{i}+\lambda_{j} \leq 2(n+1-k)+j-i\right\}$,

$w \mapsto \lambda$ is defined by $\lambda_{j}=2 n+3-k-w(j)+\#\{i<j: w(i)+w(j)>2 n+3\}$.

Recall that $w_{0}=(\overline{2}, \overline{3}, \ldots, \overline{n+1}, 1)$ whose representative indexes the full $X=\mathrm{IG}(k, 2 n+1)$ as a Schubert variety inside $\mathrm{IG}(k, 2 n+2)$. The minimal length representative for the coset of $w_{0}$ in $W^{P_{k}}$ corresponds to the $(n+1-k)$-strict partition $1^{k}:=(1,1, \ldots, 1)$ if $k<n+1$ and to $(k, 0, \ldots, 0)$ if $k=n+1$. Recall that $W^{2 n+1}=$ $W^{P_{k}} \cap\left\{w \in W: w \leq w_{0}\right\}$. The minimal length representatives for $w \in W^{2 n+1}$ are in bijection with the subset of $\Lambda_{k}^{2 n+2}$ consisting of those $(n+1-k)$-strict partitions satisfying the additional condition that if $\lambda_{k}=0$ then $\lambda_{1}=2 n+2-k$; in other words, if the first column is not full, then the first row must be full. ${ }^{1}$ Pech introduced an

\footnotetext{
${ }^{1}$ One word of caution: the Bruhat order does not translate into partition inclusion. For example, $(2 n+2-k, 0, \ldots, 0) \leq(1,1, \ldots 1)$ in the Bruhat order for $k<n+1$.
} 
equivalent indexing set, which is more convenient in the context of the odd-symplectic Grassmannians:

$$
\begin{array}{r}
\Lambda:=\left\{\lambda=\left(2 n+1-k \geq \lambda_{1} \geq \cdots \geq \lambda_{k} \geq-1\right): \lambda \text { is } n-k \text {-strict },\right. \\
\text { if } \left.\lambda_{k}=-1 \text { then } \lambda_{1}=2 n+1-k\right\} .
\end{array}
$$

Pictorially, the partitions in $\Lambda$ are obtained by removing the full first column $1^{k}$ from the partitions in $\Lambda_{k}^{2 n+2}$, regardless of whether a part equal to 0 is present.

Example 11.1.1. Let $k=5, n=7$, and $w=(1<6<\overline{8}<\overline{7}<\overline{2} \mid 3<4<5) \in W^{2 n+1}$. Then the corresponding $\lambda=\left(\lambda_{1} \geq \lambda_{2} \geq \lambda_{3} \geq \lambda_{4} \geq \lambda_{5}\right) \in \Lambda^{2 n+2}$ is given by

$$
\lambda_{1}=11, \lambda_{2}=6, \lambda_{3}=3, \lambda_{4}=3, \lambda_{5}=0 .
$$

The corresponding partition in $\Lambda$ is

$$
\left(\lambda_{1}-1 \geq \lambda_{2}-1 \geq \lambda_{3}-1 \geq \lambda_{4}-1 \geq \lambda_{5}-1\right)=(10 \geq 5 \geq 2 \geq 2 \geq-1) .
$$

Pictorially,
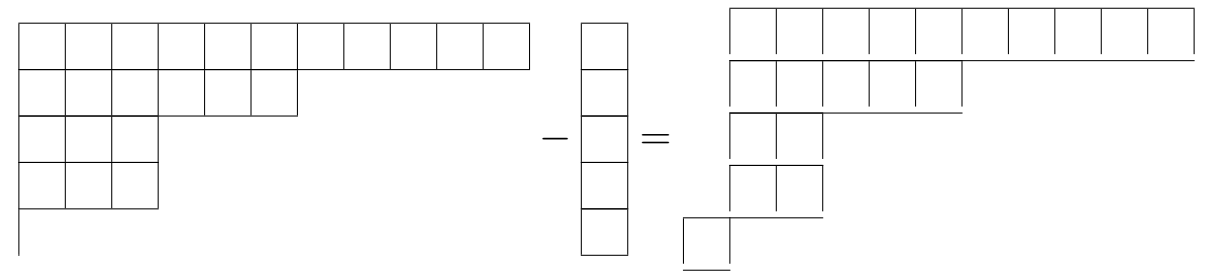

A Schubert variety $X(w)$ is included in the closed orbit $X_{c}$ of if and only if $w$ corresponds to a partition in $\lambda \in \Lambda$ such that $\lambda_{1}=2 n+1-k$. For $\lambda \in \Lambda$ define $|\lambda|=\lambda_{1}+\ldots+\lambda_{k}$. If $w$ corresponds to $\lambda$ under the bijection $W^{2 n+1} \rightarrow \Lambda$ then $\ell(w)=k(2 n+1-k)-\frac{k(k-1)}{2}-|\lambda|$, i.e. the codimension of the Schubert variety $X(w)$ in $X$ equals $|\lambda|$. Of particular interest are the elements $O_{1}$ and $O_{2}$ from Proposition 9.0.1. The corresponding partitions are:

$$
\begin{aligned}
\lambda\left(O_{1}\right)= & (2 n-k \geq 2 n-k-1 \geq 2 n-k-2 \geq \ldots \geq 2 n-2 k+2 \geq 0) ; \\
& \lambda\left(O_{2}\right)=(2 n-k+1 \geq 2 n-k-1 \geq \ldots \geq 2 n-2 k+2 \geq-1) .
\end{aligned}
$$

In order to translate the conditions from Lemma 9.0.3 in terms of partitions we need the following definition.

Definition 11.1.2. Let $\lambda=\left(\lambda_{1}, \ldots, \lambda_{k}\right)$ be a partition in $\Lambda$ such that $\lambda_{1}=2 n+1-k$.

(a) If $\lambda_{k} \geq 0$ then let $\lambda^{*}=\left(\lambda_{2} \geq \lambda_{3} \geq \cdots \geq \lambda_{k} \geq 0\right)$. If $\lambda_{k}=-1$ then $\lambda^{*}$ does not exist.

(b) If $\lambda_{2}=2 n-k$ then let $\lambda^{* *}=\left(\lambda_{1} \geq \lambda_{3} \geq \cdots \geq \lambda_{k} \geq-1\right)$. If $\lambda_{2}<2 n-k$ then $\lambda^{* *}$ does not exist. 
In both situations notice that $\left|\lambda^{*}\right|=\left|\lambda^{* *}\right|=|\lambda|-(2 n+1-k)$. As an example, if $\rho=(2 n-k+1,2 n-k, \ldots, 2 n-2 k+2)$ is the partition indexing the Schubert point, then $\lambda\left(O_{1}\right)=\rho^{*}$ and $\lambda\left(O_{2}\right)=\rho^{* *}$.

Proposition 11.1.3. Let $w \in W^{2 n+1}$ such that $w(1)=1$ and let $w \mapsto \lambda=(2 n+1-$ $\left.k, \lambda_{2}, \ldots, \lambda_{k}\right)$ be the partition in $\Lambda$ corresponding to $w$. The following hold:

(a) The partition $\lambda^{*}$ exists if and only if $w O_{1}$ is a minimal length representative in $W^{2 n+1}$. If any of these conditions is satisfied then $w O_{1} \mapsto \lambda^{*}$, thus in particular $\ell\left(w O_{1}\right)=\ell(w)+\ell\left(O_{1}\right)$.

(b) The partition $\lambda^{* *}$ exists if and only if $w O_{2}$ is a minimal length representative in $W^{2 n+1}$ and $\ell\left(w O_{2}\right)=\ell(w)+\ell\left(O_{2}\right)$. In this case $w O_{2} \mapsto \lambda^{* *}$.

Proof. Let $w=(1<2<\ldots<j<w(j+1)<\ldots<w(k) \mid w(k+1)<\ldots<w(n+1))$ where $j \geq 1, w(j+1)>j+1$ and $w(n+1) \leq n+1$ (since $w$ is a minimal length representative). Notice that either $w(k)=\overline{j+1}$ and $j+2 \leq w(k+1)$, or $w(k) \leq \overline{j+2}$ and $w(k+1)=j+1$. By the definition of $O_{1}$ and $O_{2}$, we have

$$
\begin{array}{r}
w O_{1}=(w(2), w(3), \ldots, w(k), \overline{w(k+1)} \mid 1, w(k+2), \ldots, w(n+1)) ; \\
w O_{2}=(1, w(3), \cdots, w(k), \overline{w(2)} \mid w(k+1), \cdots w(n+1)),
\end{array}
$$

as elements in $W$. Therefore $w O_{1}$ is not a minimal length representative if and only if $w(k)>\overline{w(k+1)}$, i.e. $w(k)=\overline{j+1}$ and $j+2 \leq w(k+1)$. Similarly, $w O_{2} \notin W^{2 n+1}$ if and only if $w(k)>\overline{w(2)}$.

We now proceed to prove the statement (a). If $\lambda^{*}$ exists but $w(k+1) \neq j+1$, then the preceding considerations imply that $w(k)=\overline{j+1}$. Using the bijection $W^{2 n+1} \rightarrow \Lambda$, we calculate

$$
\begin{aligned}
\lambda_{k} & =2 n+2-k-\overline{j+1}+\#\{i<k: w(i)+\overline{j+1}>2 n+3\} \\
& =j-k+\#\{i<k: w(i)>j+1\} \\
& =j-k+(k-1-j) \\
& =-1
\end{aligned}
$$

This contradicts that $\lambda_{k} \geq 0$. Therefore $w(k) \leq \overline{j+2}$ and $w(k+1)=j+1$, which means that $w O_{1} \in W^{2 n+1}$. Conversely, if $w O_{1}$ is a minimal length representative, let $w O_{1} \mapsto \mu$ under the bijection $W^{2 n+1} \rightarrow \Lambda$. Then for $1 \leq s \leq k-1$,

$$
\begin{aligned}
\mu_{s} & =2 n+2-k-w O_{1}(s)+\#\left\{i<s: w O_{1}(i)+w O_{1}(s)>2 n+3\right\} \\
& =2 n+2-k-w(s+1)+\#\{i<s: w(i+1)+w(s+1)>2 n+3\} .
\end{aligned}
$$

Since $w(1)=1, \#\{i<s: w(i+1)+w(s+1)>2 n+3\}=\#\{i<s+1: w(i)+w(s+1)>$ $2 n+3\}$, thus $\mu_{s}=\lambda_{s+1}$. We calculate $\mu_{k}$ separately:

$$
\begin{aligned}
\mu_{k} & =2 n+2-k-w O_{1}(k)+\#\left\{i<k: w O_{1}(i)+w O_{1}(k)>2 n+3\right\} \\
& =2 n+2-\overline{j+1}+\#\{i<k: w(i+1)+\overline{j+1}>2 n+3\} \\
& =j-k+\#\{i<k: w(i+1)>j+1\} \\
& =j-k+(k-j) \\
& =0 .
\end{aligned}
$$


Then $\mu=\lambda^{*}$, and in particular the length condition is satisfied.

We now prove (b). We first observe that $\lambda_{2}=2 n+2-k-w(2)$. Then $\lambda^{* *}$ exists if and only if $\lambda_{2}=2 n-k$, i.e. $w(2)=2$. Then clearly $w(k)<\overline{w(2)}$, therefore $w O_{2} \in W^{2 n+1}$. Let $w O_{2} \mapsto \mu$. As before we calculate $\mu_{1}=2 n+1-k, \mu_{s}=\lambda_{s+1}$ for $2 \leq s \leq k-1$, and that $\mu_{k}=w(2)-3=-1$. This proves one implication. For the converse, we notice that once $w O_{2} \in W^{2 n+1}$, same calculations show that $\mu_{1}=2 n+1-k$ and $w(1)=1$ to obtain that $\mu_{s}=\lambda_{s+1}$ for $2 \leq s \leq k-1$ (the condition $w(2)=2$ is not used in these). The length condition on $\ell\left(w O_{2}\right)$ implies that $|\lambda|-|\mu|=2 n+1-k$, which forces $\mu_{k}=-1$, thus $\mu=\lambda^{* *}$ and the proposition is proved.

\subsection{The equivariant quantum Chevalley formula}

To formulate the equivariant quantum Chevalley formula we will first recall the (nonquantum) equivariant Chevalley formula for $X=\operatorname{IG}(k, 2 n+1)$. This is due to Pech [55], but for the convenience of the reader we briefly recall the main steps. (Pech works in the non-equivariant setting, and occasionally we need to add few extra arguments to extend her proofs to the equivariant setting.) In a nutshell, Pech uses the embedding $\iota: X \rightarrow \mathrm{IG}(k, 2 n+2)$ to reduce the calculation to the Chevalley formula $\mathrm{H}^{*}(\mathrm{IG}(k, 2 n+2))$. Since $\mathrm{IG}(k, 2 n+2)$ is a homogeneous case, the classical work of Chevalley [18], and its equivariant generalization (see e.g. [40]), give the formula with Schubert classes indexed in terms of the Weyl group representatives. A further translation is needed in order to replace Weyl group representatives by partitions; this was recently obtained by Buch, Kresch and Tamvakis [13] who actually proved a more general (but non-equivariant) Pieri rule.

In this section we will use the notation $X(\lambda)$ to denote the Schubert variety in $X$ and $Y\left(\lambda+1^{k}\right)$ to denote the same Schubert variety, but now regarded in $\operatorname{IG}(k, 2 n+2)$. The notation is consistent with the fact that the partitions in $\Lambda^{2 n+2}$ are obtained from the "odd-symplectic partitions" $\lambda \in \Lambda$ by adding one box to each row. We denote by $X($ Div $)$ respectively $Y($ Div $)$ the Schubert divisors in $X$ and $\operatorname{IG}(k, 2 n+2)$. Pech proved that in $\mathrm{H}^{*}(X)$ there is an equality $\iota^{*}[Y(D i v)]=[X(D i v)]$. Therefore in the equivariant cohomology $\iota^{*}[Y(\text { Div })]_{T}=[X(\text { Div })]_{T}+C(T)$, where $C(T) \in H_{T}^{2}(p t)$ is a homogeneous linear form. After localization at the point $w_{0} W_{P}$ (the torus-fixed point in the open Schubert $X \subset \mathrm{IG}(k, 2 n+2))$, and using that $w_{0} W_{P} \notin X($ Div $)$ we obtain that $C(T)=\iota_{w_{0}}^{*}[Y(D i v)]_{T}$ (the localization of $[Y(D i v)]_{T}$ at $\left.w_{0} W_{P}\right)$. Consider the expansions

$$
\begin{aligned}
{[X(\text { Div })]_{T} \cup[X(\lambda)]_{T} } & =\sum_{\mu \in \Lambda} c_{D i v, \lambda}^{\mu}[X(\mu)]_{T} \in \mathrm{H}_{T}^{*}(X) ; \\
{[Y(\text { Div })]_{T} \cup[Y(\lambda+1)]_{T} } & =\sum_{\mu \in \Lambda} \tilde{c}_{D i v, \lambda}^{\mu}[Y(\mu+1)]_{T} \in \mathrm{H}_{T_{2 n+2}}^{*}(\operatorname{IG}(k, 2 n+2)),
\end{aligned}
$$

where $c_{D i v, \lambda}^{\mu}, \tilde{c}_{D i v, \lambda}^{\mu} \in \mathrm{H}_{T_{2 n+2}}^{*}(p t)=\mathrm{H}_{T}^{*}(p t)$ are homogeneous polynomials. Notice that $\iota_{*}[X(\lambda)]_{T}=[Y(\lambda+1)]_{T}$ therefore the product $[Y(\text { Div })]_{T} \cup[Y(\lambda+1)]_{T}$ will only contain 
cohomology classes supported on $\mathrm{H}_{T}^{*}(X)$, of the form $\iota_{*}[X(\mu)]_{T}$. In particular, since $w_{0} W_{P}$ corresponds to the partition $1^{k} \in \Lambda^{2 n+2}$,

$$
C(T)=\tilde{c}_{D i v, 1^{k}}^{1^{k}}=t_{1}-t_{k+1} .
$$

We apply $\iota_{*}$ to both sides of (11.2) and the projection formula to obtain

$$
\begin{aligned}
\iota_{*}\left([X(D i v)]_{T} \cup[X(\lambda)]_{T}\right) & =\iota_{*}\left(\left(\iota^{*}[Y(D i v)]_{T}-C(T)\right) \cup[X(\lambda)]_{T}\right) \\
& =\left([Y(D i v)]_{T}-C(T)\right) \cup[Y(\lambda+1)]_{T} \\
& =[Y(D i v)]_{T} \cup[Y(\lambda+1)]_{T}-C(T)[Y(\lambda+1)]_{T}
\end{aligned}
$$

It follows from this that

$$
c_{D i v, \lambda}^{\mu}= \begin{cases}\tilde{c}_{D i v, \lambda}^{\mu} & \text { if } \lambda \neq \mu ; \\ \tilde{c}_{D i v, \lambda}^{\lambda}-\tilde{c}_{D i v, 1^{k}}^{1^{k}} & \text { if } \lambda=\mu .\end{cases}
$$

Notice in particular that if $\lambda \neq \mu$, the coefficients $\tilde{c}_{D i v, \lambda}^{\mu}$ are integers. We recall next the formula for these integers obtained in [13].

Definition 11.2.1. Let $\lambda \in \Lambda^{2 n+2}$ represented as a Young diagram. The box in row $r$ and column $c$ of $\lambda$ is $(n+1-k)$ - related to the box in row $r^{\prime}$ and column $c^{\prime}$ if

$$
|c-n+k-2|+r=\left|c^{\prime}-n+k-2\right|+r^{\prime} .
$$

Given $\lambda, \mu \in \Lambda^{2 n+2}$ with $\lambda \subset \mu$, the skew diagram $\mu / \lambda$ is called a horizontal strip (resp. vertical) strip if it does not contain two boxes in the same column (resp. row).

Following [13, Definition 1.3] we say $\lambda \rightarrow \mu$ for any $n+1-k$-strict partitions $\lambda, \mu$ if $\mu$ can be obtained by removing a vertical strip from the first $n+1-k$ columns of $\lambda$ and adding a horizontal strip to the result, so that

1. if one of the first $n+1-k$ columns of $\mu$ has the same number of boxes as the same column of $\lambda$, then the bottom box of this column is $n+1-k$-related to at most one box of $\mu \backslash \lambda$; and

2. if a column of $\mu$ has fewer boxes than the same column of $\lambda$, the removed boxes and the bottom box of $\mu$ in this column must each be $n+1-k$-related to exactly one box of $\mu \backslash \lambda$, and these boxes of $\mu \backslash \lambda$ must all lie in the same row.

If $\lambda \rightarrow \mu$, we let $\mathbb{A}$ be the set of boxes of $\mu \backslash \lambda$ in columns $n+2-k$ through $2 n+1-k$ which are not mentioned in (1) or (2). Then define $N(\lambda, \mu)$ to be the number of connected components of $\mathbb{A}$ which do not have a box in column $n+2-k$. Here two boxes are connected if they share at least a vertex.

Example 11.2.2. Let $n=4$ and $k=3$. We will fill each box of the full staircase $\lambda=(6 \geq 5 \geq 4)$ with $|c-n+k-2|+r$. So we have

\begin{tabular}{|l|l|l|l|l|l|}
\hline 3 & 2 & 1 & 2 & 3 & 4 \\
\hline 4 & 3 & 2 & 3 & 4 & \multicolumn{1}{|c}{} \\
\cline { 1 - 4 } 5 & 4 & 3 & 4 & \multicolumn{1}{|c}{. } \\
\cline { 1 - 3 }
\end{tabular}


Boxes that share the same number are $n+1-k$-related. Pictorially, the related boxes are situated on diagonals which are "mirror symmetric" with respect to a vertical axis on the $n+2-k$ column.

Example 11.2.3. Let $n=k=2$ and $\lambda=(2 \geq 1)$ and $\mu=(3 \geq 1)$. The partition $\mu$ is obtained by adding a box to the first row $\lambda$. So $\lambda \rightarrow \mu$. Filling the boxes of $\lambda$ with $|c-n+k-2|+r$ yields

$$
\begin{array}{|l|l|}
\hline 2 & 1 \\
\hline 3 &
\end{array}
$$

and filling the boxes of $\mu$ with $|c-n+k-2|+r$ yields

$$
\begin{array}{|l|l|l|}
\hline 2 & 1 & 2 \\
\hline 3 & \multicolumn{2}{|l}{} \\
\hline
\end{array}
$$

Since the box in position $(r, c)=(1,3)$ is not mentioned in (1) or (2) of Definition 11.2.1 we have that $\mathbb{A}$ contains one element. Thus, $N(\lambda, \mu)=1$. One can also see [13] for an example.

Combining theorem 10.0.3, proposition 11.1.3, and equation (11.3) above, together with the formulation of the Chevalley rule for $\mathrm{H}^{*}(\mathrm{IG}(k, 2 n+2)$ obtained in $[13$, Theorem 1.1] yields the equivariant quantum Chevalley formula (see also [63]):

Theorem 11.2.4. Let $\lambda \in \Lambda$ be ant $n-k$ strict partition. Then the following equality holds in the equivariant quantum ring $\mathrm{QH}_{T}^{*}(\mathrm{IG}(k, 2 n+1))$ :

$$
\begin{aligned}
{[X(D i v)]_{T} \star[X(\lambda)]_{T} } & =\left(\sum_{\left(\lambda+1^{k}\right) \rightarrow\left(\mu+1^{k}\right),|\mu|=|\lambda|+1} 2^{N\left(\lambda+1^{k}, \mu+1^{k}\right)}[X(\mu)]_{T}\right) \\
& +\left(\omega_{k}+\sum_{j=1}^{k} t_{w_{j}}-\left(t_{1}-t_{k+1}\right)\right)[X(\lambda)]_{T}+q\left[X\left(\lambda^{*}\right)\right]_{T}+q\left[X\left(\lambda^{* *}\right)\right]_{T},
\end{aligned}
$$

where $w(j)=2 n+2-k-\lambda_{j}+\#\left\{i<j: \lambda_{i}+\lambda_{j} \leq 2(n-k)+j-i\right\}$. If $\lambda^{*}$ or $\lambda^{* *}$ do not exist then the corresponding quantum term is omitted.

Example 11.2.5. Consider $n=4$ and $k=3$. For $\lambda=(6,5,1)$ our quantum components are indexed by $\lambda^{*}=(5,1,0)$ and $\lambda^{* *}=(6,1,-1)$. The corresponding diagrams, and two additional examples are exhibited in the table below. 


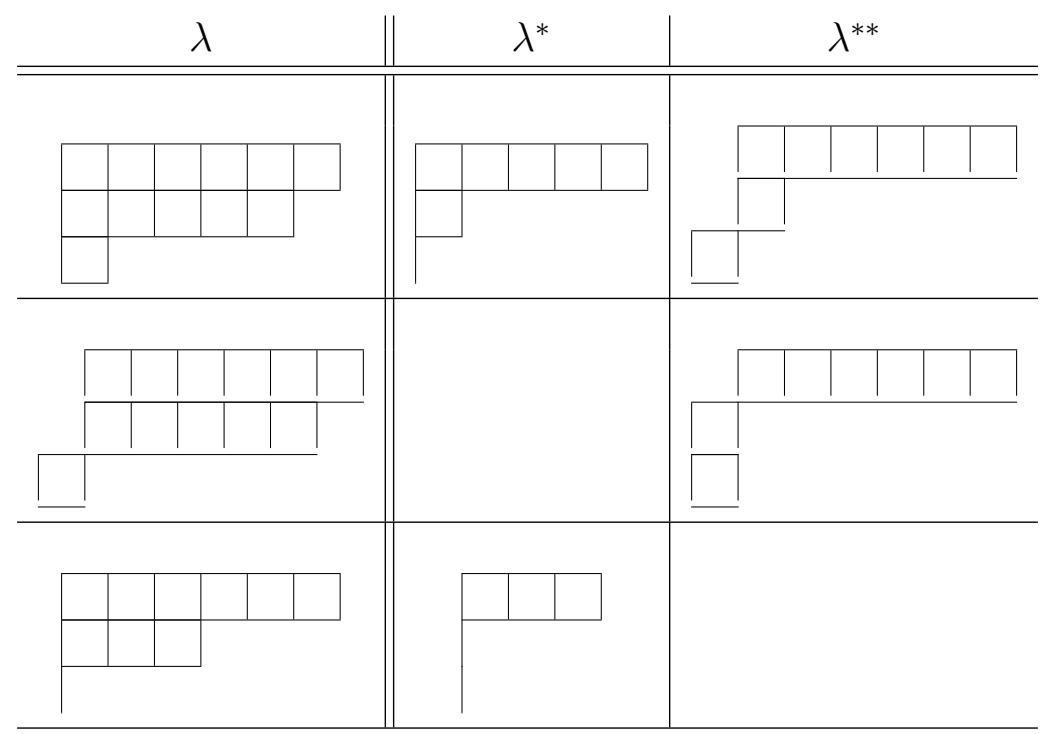

\subsubsection{The EQ Chevalley formula for $\operatorname{IG}(3,7)$}

The Chevalley formula for $\mathrm{QH}_{T}^{*}(\mathrm{IG}(3,7))$ is

$$
\begin{aligned}
& {[\mathrm{X}(1)] \star[X(0)]=[\mathrm{X}(1)]} \\
& {[\mathrm{X}(1)] \star \quad[X(1)]=\left(2 t_{4}\right)[X(1)]+[\mathrm{X}(4,-1,-1)]+2[X(2)]} \\
& {[\mathrm{X}(1)] \star[X(4,-1,-1)]=\left(t_{1}+t_{4}\right)[X(4,-1,-1)]+[X(4,0,-1)]} \\
& {[\mathrm{X}(1)] \star[X(2)]=\left(2 t_{3}\right)[X(2)]+[X(4,0,-1)]+[X(2,1)]+2[X(3)]} \\
& {[\mathrm{X}(1)] \star[X(4,0,-1)]=\left(t_{1}+t_{3}\right)[X(4,0,-1)]+[X(4,1,-1)]+[X(4)]} \\
& {[\mathrm{X}(1)] \star[X(2,1)]=2\left(t_{3}+t_{4}\right)[X(2,1)]+[X(4,1,-1)]+2[X(3,1)]} \\
& {[\mathrm{X}(1)] \star[X(3)]=\left(2 t_{2}\right)[X(3)]+[X(3,1)]+[X(4)]} \\
& {[\mathrm{X}(1)] \star[X(4,1,-1)]=\left(t_{1}+t_{3}+2 t_{4}\right)[X(4,1,-1)]+[X(4,2,-1)]+[X(4,1)]} \\
& {[\mathrm{X}(1)] \star \quad[X(3,1)]=2\left(t_{2}+t_{4}\right)[X(3,1)]+[X(4,1)]+2[X(3,2)]} \\
& {[\mathrm{X}(1)] \star[X(4)]=\left(t_{1}+t_{2}\right)[X(4)]+[X(4,1)]+q} \\
& {[\mathrm{X}(1)] \star[X(4,2,-1)]=\left(t_{1}+2 t_{3}+t_{4}\right)[X(4,2,-1)]+[X(4,2)]+2[X(4,3,-1)]} \\
& [\mathrm{X}(1)] \star \quad \star X(3,2)]=2\left(t_{2}+t_{3}\right)[X(3,2)]+[X(3,2,1)]+[X(4,2)] \\
& {[\mathrm{X}(1)] \star[X(4,1)]=\left(t_{1}+t_{2}+2 t_{4}\right)[X(4,1)]+[X(4,3,-1)]+2[X(4,2)]+q[X(1)]} \\
& {[\mathrm{X}(1)] \star[X(4,3,-1)]=\left(t_{1}+2 t_{2}+t_{4}\right)[X(4,3,-1)]+[X(4,3)]+q[X(4,-1,-1)]} \\
& {[\mathrm{X}(1)] \star[X(3,2,1)]=2\left(t_{2}+t_{3}+t_{4}\right)[X(3,2,1)]+[X(4,2,1)]} \\
& {[\mathrm{X}(1)] \star[X(4,2)]=\left(t_{1}+t_{2}+2 t_{3}\right)[X(4,2)]+[X(4,2,1)]+[X(4,3)]+q[X(2)]} \\
& {[\mathrm{X}(1)] \star[X(4,2,1)]=\left(t_{1}+t_{2}+2 t_{3}+2 t_{4}\right)[X(4,2,1)]+[X(4,3,1)]+q[X(2,1)]} \\
& {[\mathrm{X}(1)] \star[X(4,3)]=\left(t_{1}+2 t_{2}+t_{3}\right)[X(4,3)]+[X(4,3,1)]+q[X(4,0,-1)]+q[X(3)]} \\
& {[\mathrm{X}(1)] \star[X(4,3,1)]=\left(t_{1}+2 t_{2}+t_{3}+2 t_{4}\right)[X(4,3,1)]+[X(4,3,2)]} \\
& +q[X(4,1,-1)]+q[X(3,1)] \\
& {[\mathrm{X}(1)] \star \quad[X(4,3,2)]=\left(t_{1}+2 t_{2}+2 t_{3}+t_{4}\right)[X(4,3,2)]+q[X(4,2,-1)]+q[X(3,2)]}
\end{aligned}
$$




\section{Chapter 12}

\section{Calculating $\mathrm{QH}_{T}^{*}(\mathrm{IG})$}

The class of the divisor $[X(1)]$ does not generate the ring $\mathrm{QH}_{T}^{*}(\mathrm{IG}(k, 2 n+1))$. However, it is sufficient to calculate the coefficients in $\mathrm{QH}_{T}^{*}(\mathrm{IG}(k, 2 n+1))$ by using just the EQ Chevalley formula. Such a calculations are performed by Okounkov in [54]; Molev and Sagan in [52]; Knutson and Tao in the equivariant case in [37]; Mihalcea in the equivariant quantum case in [50,51]; and by Buch, Chaput, Mihalcea, and Perrin in the equivariant quantum $K$-theory of cominuscule varieties in [8]. The calculation of a recursive formula is the goal of the chapter. We need to introduce a few notations that compute $\lambda$ given $\lambda^{*}$ and $\lambda^{* *}$. If

$$
\mu=\left(\mu_{1} \geq \cdots \geq \mu_{k-1} \geq 0\right)
$$

where $\mu_{1}<2 n+1-k$ then define

$$
\mu^{+}=\left(2 n+1-k \geq \mu_{1} \geq \cdots \geq \mu_{k-1}\right) .
$$

If

$$
\mu=\left(2 n+1-k \geq \mu_{2} \geq \cdots \geq \mu_{k-1} \geq-1\right)
$$

where $\mu_{2}<2 n-k$ then define

$$
\mu^{++}=\left(2 n+1-k \geq 2 n-k \geq \cdots \geq \mu_{k-1}\right) .
$$

The next proposition introduces an important recursive formula which allows us to compute the the entire equivariant quantum cohomology ring.

Proposition 12.0.1. The coefficients in $\mathrm{QH}_{T}^{*}(\mathrm{IG}(k, 2 n+1))$ satisfy the following equation:

$$
\begin{aligned}
\left(c_{(1), \mu}^{\mu, 0}-c_{(1), \lambda}^{\lambda, 0}\right) c_{\lambda, \zeta}^{\mu, d} & =\sum_{\substack{\left(\lambda+1^{k}\right) \rightarrow\left(\delta+1^{k}\right) \\
|\delta|=|\lambda|+1}} 2^{N\left(\lambda+1^{k}, \delta+1^{k}\right)} c_{\delta, \zeta}^{\mu, d}-\sum_{\substack{\left(\gamma+1^{k}\right) \rightarrow\left(\mu+1^{k}\right) \\
|\mu|=|\gamma|+1}} 2^{N\left(\gamma+1^{k}, \mu+1^{k}\right)} c_{\lambda, \zeta}^{\gamma, d} \\
& +c_{\lambda^{*}, \zeta}^{\mu, d-1}+c_{\lambda^{* *}, \zeta}^{\mu, d-1}-c_{\lambda, \zeta}^{\mu^{+}, d-1}-c_{\lambda, \zeta}^{\mu^{++}, d-1}
\end{aligned}
$$

for any permutations $\lambda, \zeta, \mu \in \Lambda$ and any nonnegative integer $d$. The third (resp. fourth, fifth, and sixth) term are omitted if $\lambda^{*}$ is not defined (resp. $\lambda^{* *}, \mu^{+}$, and $\mu^{++}$ are not defined). These four terms are omitted if $d=0$. 
Proof. We will make use of the EQ Chevalley Rule and the associative property $[X(1)] *([X(\lambda)] *[X(\zeta)])=([X(1)] *[X(\lambda)]) *[X(\zeta)]$. We also have $[X(\lambda)] *[X(1)]=$ $[X(1)] *[X(\lambda)]$. Thus,

$$
\begin{aligned}
& {[X(1)] *([X(\lambda)] *[X(\zeta)])=[X(1)] *\left(\sum_{d, \sigma} q^{d} c_{\lambda, \zeta}^{\sigma, d}[X(\sigma)]\right) } \\
= & \sum_{d, \sigma} q^{d} c_{\lambda, \zeta}^{\sigma, d}\left(\sum_{\substack{\left(\sigma+1^{k}\right) \rightarrow\left(\beta+1^{k}\right) \\
|\sigma|=|\beta|+1}} 2^{N\left(\sigma+1^{k}, \beta+1^{k}\right)}[X(\beta)]+c_{1, \sigma}^{\sigma}[X(\sigma)]+q\left[X\left(\sigma^{*}\right)\right]+q\left[X\left(\sigma^{* *}\right)\right]\right)
\end{aligned}
$$

and

$$
\begin{aligned}
& ([X(1)] *[X(\lambda)]) *[X(\zeta)] \\
= & \left(\sum_{\substack{\left(\lambda+1^{k}\right) \rightarrow\left(\delta+1^{k}\right) \\
|\delta|=|\lambda|+1}} 2^{N\left(\lambda+1^{k}, \delta+1^{k}\right)}[X(\delta)]+c_{1, \lambda}^{\lambda}[X(\lambda)]+q\left[X\left(\lambda^{*}\right)\right]+q\left[X\left(\lambda^{* *}\right)\right]\right) *[X(\zeta)] \\
= & \sum_{\substack{\left(\lambda+1^{k}\right) \rightarrow\left(\delta+1^{k}\right) \\
|\lambda|=|\delta|+1}} 2^{N\left(\lambda+1^{k}, \delta+1^{k}\right)}\left(\sum_{\alpha_{1}, d_{1}} q^{d_{1}} c_{\delta, \zeta}^{\alpha_{1}, d_{1}}\left[X\left(\alpha_{1}\right)\right]\right)+c_{1, \lambda}^{\lambda}\left(\sum_{d_{2}, \alpha_{2}} q^{d_{2}} c_{\lambda, \zeta}^{\alpha_{2}, d_{2}}\left[X\left(\alpha_{2}\right)\right]\right) \\
+ & q\left(\sum_{\alpha_{3}, d_{3}} q^{d_{3}} c_{\lambda^{*}, \zeta}^{\alpha_{3}, d_{3}}\left[X\left(\alpha_{3}\right)\right]\right)+q\left(\sum_{\alpha_{4}, d_{4}} q^{d_{4}} c_{\lambda^{* *}, \zeta}^{\alpha_{4}, d_{4}}\left[X\left(\alpha_{4}\right)\right]\right)
\end{aligned}
$$

Collecting the coefficient of $q^{d}[X(\mu)]$ from both sides give

$$
\begin{aligned}
& \sum_{\substack{\left(\epsilon+1^{k}\right) \rightarrow\left(\mu+1^{k}\right) \\
|\mu|=|\epsilon|+1}} 2^{N\left(\epsilon+1^{k}, \mu+1^{k}\right)} c_{P, S}^{Z, d}+c_{\lambda, \zeta}^{\mu^{+}, d-1}+c_{\lambda, \zeta}^{\mu^{++}, d-1}+c_{1, \mu}^{\mu} c_{\lambda, \zeta}^{\mu, d} \\
= & \sum_{\substack{\left(\lambda+1^{k}\right) \rightarrow\left(\delta+1^{k}\right) \\
|\delta|=|\lambda|+1}} 2^{N\left(\lambda+1^{k}, \delta+1^{k}\right)} c_{\delta, \zeta}^{\mu, d}+c_{\lambda^{*}, \zeta}^{\mu, d-1}+c_{\lambda^{* *}, \zeta}^{\mu, d-1}+c_{1, \lambda}^{\lambda} c_{\lambda, \zeta}^{\mu, d}
\end{aligned}
$$

The stated formula in the Proposition is a consequence of rearranging terms.

\subsection{Notation}

We will define some notation which will be used in the up coming results. Let $F_{\mu, \lambda}$ denote the polynomial

$$
F_{\mu, \lambda}=c_{1, \mu}^{\mu, 0}-c_{1, \lambda}^{\lambda, 0}
$$


Let $\alpha$ and $\lambda$ be two partitions such that $\lambda$ is less than $\alpha$ in the Bruhat order. Define a rational function $R(\Lambda)$, the fraction field of $\Lambda\left(=\mathbb{Z}\left[t_{1}, \cdots, t_{n+1}\right]\right)$, as follows:

$$
R_{\lambda, \alpha}=\left\{\begin{array}{cc}
\sum \Pi_{i=0}^{l} \frac{1}{F_{\lambda, \alpha}(i)} & \text { if } \lambda \neq \alpha \\
1 & \text { if } \alpha=\lambda
\end{array}\right.
$$

where $l=|\lambda|-|\alpha|$ and the sum is over all chains of permutations

$$
\lambda=\alpha^{(l)} \leftarrow_{i_{l}} \alpha^{(l-1)} \leftarrow_{i_{l-1}} \cdots \leftarrow_{i_{1}} \alpha^{(1)} \leftarrow_{i_{1}} \alpha^{(0)}=\alpha
$$

where $i_{j}$ is either 1 or 2 for all $j$.

Let $(\lambda, \mu)$ and $(\delta, \epsilon)$ be two pairs of permutations in $\Lambda$. Define

$$
(\lambda, \mu)<_{1}(\delta, \epsilon)
$$

if $\lambda \rightarrow \delta$ and $\mu=\epsilon$ and define

$$
(\lambda, \mu)<_{2}(\delta, \epsilon)
$$

if $\delta=\lambda$ and $\epsilon \rightarrow \mu$. Notice in each case that $|\delta|-|\epsilon|=|\lambda|-|\mu|+1$. Finally rearranging the formula in Proposition 12.0.1 we have

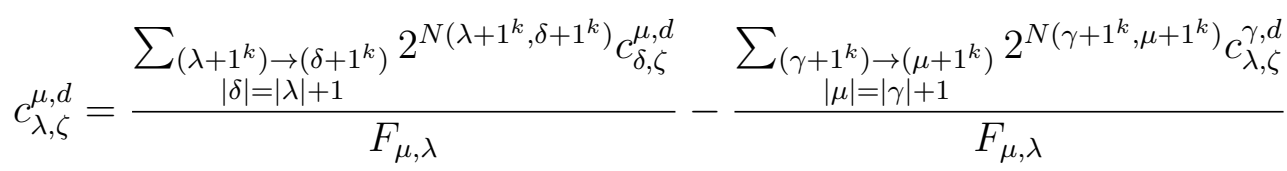

$$
\begin{aligned}
& +\frac{c_{\lambda^{*}, \zeta}^{\mu, d-1}}{F_{\mu, \lambda}}+\frac{c_{\lambda^{* *}, \zeta}^{\mu, d-1}}{F_{\mu, \lambda}}-\frac{c_{\lambda, \zeta}^{\mu^{+}, d-1}}{F_{\mu, \lambda}}-\frac{c_{\lambda, \zeta}^{\mu^{++}, d-1}}{F_{\mu, \lambda}}
\end{aligned}
$$

\subsection{Two formulae}

We will now state and prove the analog of Proposition 6.1 from [50].

Proposition 12.2.1. Let $\lambda, \zeta, \mu$ be partitions such that $\lambda$ is not included in $\mu$ and let $d$ be a nonnegative integer. Then

$$
c_{\lambda, \zeta}^{\mu, d}=E_{\lambda, \zeta, \mu}(d)
$$

where $E_{\lambda, \zeta, \mu}(d)$ is a linear homogeneous expression in EQ coefficients of degree $d-1$ with coefficients in $R(\Lambda)$, the fraction field of $\Lambda\left(=\mathbb{Z}\left[t_{1}, \cdots, t_{n+1}\right]\right)$. If $d=0$ then $c_{\lambda, \zeta}^{\mu, 0}=E_{\lambda, \zeta, \mu}(0)=0$.

Proof. The proof will be by induction on the difference $|\lambda|-|\mu| \leq \operatorname{dim} \operatorname{IG}(k, 2 n+1)$. 
Base case: Let $|\lambda|-|\mu|=\operatorname{dim} \operatorname{IG}(k, 2 n+1)$. Then $\mu=(0)$ and $\lambda=($ full). Consider $c_{\lambda, \zeta}^{\mu, d}$. The first two terms of equation (12.1) vanish when applied to $c_{\lambda, \zeta}^{\mu, d}=$ $c_{(\text {full }), \zeta}^{(0), d}$. So we have

$$
E_{(f u l l), \zeta,(0)}(d)=\frac{1}{F_{(0),(f u l l)}}\left(c_{(f u l l)^{*}, \zeta}^{(0), d-1}+c_{(f u l l)^{* *}, \zeta}^{(0), d-1}-c_{(f u l l), \zeta}^{(0)^{+}, d-1}-c_{(f u l l), \zeta}^{(0)^{++}, d-1}\right) .
$$

This establishes the base case.

Let $|\lambda|-|\mu|<\operatorname{dim} \operatorname{IG}(k, 2 n+1)$ such that $\lambda$ is not included in $\mu$. Applying equation (12.1) to $c_{\lambda, \zeta}^{\mu, d}$ yields coefficients of degree $d-1$ and coefficients $c_{\delta, \zeta}^{\epsilon, d}$ with $(\lambda, \mu)<_{i}(\delta, \epsilon)$ for $i=1,2$. It is enough to show that each coefficients $c_{\delta, \zeta}^{\epsilon, d}$ is equal to a $R(\Lambda)$-linear homogeneous expression $E_{\delta, \zeta, \epsilon}(d)$ in coefficients of degree $d-1$. Since $\lambda$ is greater than $\delta$ and $\epsilon$ is greater than $\mu$ in the Bruhat order, and $\lambda$ is not greater than me $\mu$ then $\delta$ is not greater than $\epsilon$. Finally, $|\delta|-|\epsilon|=|\lambda|-|\mu|+1$ and the induction hypothesis applied to $c_{\delta, \zeta}^{\epsilon, d}$ implies the desired result for $d>0$. The same proof will work for $d=0$. Also, $c_{\lambda, \zeta}^{\mu, 0}=E_{\lambda, \zeta, \mu}(0)=0$ since the last four terms of equation (12.1) are ignored.

Proposition 12.2.2. The EQ coefficient $c_{\alpha, \lambda}^{\lambda, d}$ satisfy the following formula:

$$
c_{\alpha, \lambda}^{\lambda, d}=R_{\lambda, \alpha} c_{\lambda, \lambda}^{\lambda, d}+E_{\lambda, \alpha}^{\prime}(d)
$$

where $E_{\lambda, \alpha}^{\prime}(d)$ is an $R(\Lambda)$-linear homogeneous expression in $E Q$ coefficients of degree $d-1$. If $d=0$ then $E_{\lambda, \alpha}^{\prime}=0$. Moreover, for any such $\lambda$ and $\alpha, R_{\lambda, \alpha}$ is a different from 0 .

Proof. Since $\alpha$ is included in $\lambda$ then $|\lambda|-|\alpha| \geq 0$. We will use induction on $|\lambda|-|\alpha|$.

For the base case suppose $|\lambda|-|\alpha|=0$ then $\lambda=\alpha$. This established the base case.

Let $|\lambda|-|\alpha|>0$. Since $\alpha$ is different from $\lambda$ we can apply equation (12.1) to $c_{\alpha, \lambda}^{\lambda, d}$. In equation (12.1) the first term contain coefficients $c_{\delta, \lambda}^{\epsilon, d}$ with $(\alpha, \lambda)<_{1}(\delta, \epsilon)$. Also, the second term of equation (12.1) contain coefficients $c_{\delta, \lambda}^{\epsilon, d}$ with $(\alpha, \lambda)<_{2}(\delta, \epsilon)$.

Consider a coefficient from the second term of equation (12.1). Then $\delta=\alpha$ and $\epsilon \rightarrow \lambda$. So, $\lambda$ is not greater than $\epsilon$. So we can conclude from Proposition 12.2.1

$$
c_{\alpha, \lambda}^{\epsilon, d}=E_{\alpha, \lambda, \epsilon}(d) .
$$

Consider a coefficient from the first term of equation (12.1). Then $\lambda=\epsilon$ and $\alpha \rightarrow \delta$. If $\delta$ is not greater than $\lambda$ then by Proposition 12.2.1

$$
c_{\delta, \lambda}^{\epsilon, d}=c_{\delta, \lambda}^{\lambda, d}=E_{\delta, \lambda, \lambda}(d) .
$$

If $\delta$ is greater than $\lambda$ then by the induction hypothesis we have

$$
c_{\delta, \lambda}^{\epsilon, d}=c_{\delta, \lambda}^{\lambda, d}=R_{\lambda, \delta} c_{\lambda, \lambda}^{\lambda, d}+E_{\lambda, \delta}^{\prime}(d) .
$$


The following is a consequence of combining equations (12.1), (12.2), (12.3), (12.4), and $R_{\lambda, \alpha}=\frac{1}{F_{\lambda, \alpha}} \sum R_{\lambda, \delta}$, where the sum is over all $\delta$ such that $\alpha \rightarrow \delta$ and $\delta$ is greater than $\lambda$.

$$
\begin{aligned}
c_{\alpha, \lambda}^{\lambda, d} & =\frac{1}{F_{\lambda, \alpha}}\left(\sum\left(R_{\lambda, \delta} c_{\lambda, \lambda}^{\lambda, d}+E_{\lambda, \delta}^{\prime}(d)\right)+\sum E_{\delta, \lambda, \lambda}(d)\right) \\
& +\frac{1}{F_{\lambda, \alpha}}\left(\sum_{\substack{\left(\epsilon+1^{k}\right) \rightarrow\left(\lambda+1^{k}\right) \\
|\lambda|=|\epsilon|+1}} E_{\alpha, \lambda, \epsilon(d)}\right)+\frac{c_{\lambda^{*}, S}^{Q, d-1}+c_{\lambda^{* *}, \zeta}^{\mu, d-1}-c_{\lambda, \zeta}^{\mu^{+}, d-1}-c_{\lambda, \zeta}^{\mu^{++}, d-1}}{F_{\mu, \lambda}} \\
& =R_{\lambda, \alpha} c_{\lambda, \lambda}^{\lambda, d}+E_{\lambda, \alpha}^{\prime}(d) .
\end{aligned}
$$

The first sum is over all $\delta$ such that $\alpha \rightarrow \delta$ and $\delta$ is greater than $\lambda$. The second sum is over all $\delta$ such that $\alpha \rightarrow \delta$ and $\delta$ is not greater than $\lambda$. We find $E_{\lambda, \alpha}^{\prime}(d)$ by collecting terms with coefficients of degree $d-1$.

The expression $F_{\lambda, \alpha^{(j)}}$ is a linear homogeneous polynomial in variables $t_{1}-t_{2}, \cdots, t_{n}-$ $t_{n+1}, 2 t_{n+1}$ with positive coefficients. Indeed, $F_{\lambda, \alpha^{(j)}}=w\left(t_{1}+\cdots+t_{k}\right)-v\left(t_{1}+\cdots+t_{k}\right)$ where $\lambda \mapsto w \in W^{2 n+1}$ and $\alpha^{(j)} \mapsto v \in W^{2 n+1}$ using the bijection in $\Lambda \rightarrow W^{2 n+1}$. Observe that $\lambda$ is less than $\alpha^{(j)}$ in the Bruhat order so we have that $w$ is less than $v$ in the Bruhat order. The inequality $w(i) \leq v(i)$ holds for $1 \leq i \leq k$ thus $t_{w(i)}-t_{v(j)}$ is a positive root or zero. The claim follows.

Therefore, $R_{\lambda, \alpha}(t)$ cannot equal zero since there cannot be cancellation.

\subsection{An algorithm to compute the EQ coefficients}

Theorem 12.3.1. The EQ coefficients are determined (algorithmically) by the following formulae

1. $c_{(0),(0)}^{(0), d}=0$ unless $d=0$, then it is equal to 1.

2. (commutativity) $c_{\lambda, \zeta}^{\mu, d}=c_{\zeta, \lambda}^{\mu, d}$ for al partitions $\lambda, \zeta$, and $\mu$.

3. (EQ Chevalley) The coefficients $c_{1, \lambda}^{\mu}$ from equation (1.2.3) for all partitions $\lambda$ and $\mu$.

4. Equation (12.1) for all partitions $\lambda, \zeta, \mu$ such that $\lambda$ is different from $\mu$.

Proof. Step 1: The coefficents $c_{\lambda, \zeta}^{\mu, 0}$ can be found algorithmically for all partitions $\lambda, \zeta$, and $\mu$. See $[37,52,54]$.

Let $d>0$ and for the inductive hypothesis assume that the EQ coefficients of degree $d-1$ are known.

Step 2: We will now compute $c_{\lambda, \lambda}^{\lambda, d}$ for each permutation $\lambda$. If $\lambda=(0)$ or $\lambda=1$ then the coefficient $c_{\lambda, \lambda}^{\lambda, d}$ is given by 1 and 2. Assume that $|\lambda| \geq 2$. By 3, the EQ 
coefficients $c_{1, \lambda}^{\lambda, d}=c_{\lambda, 1}^{\lambda, d}$ vanish. By Proposition 12.2.2 we have

$$
c_{\lambda, \lambda}^{\lambda, d}=-\frac{E_{1, \lambda}^{\prime}(d)}{R_{1, \lambda}}
$$

Finally, by induction on $d$ we have $E_{1, \lambda}^{\prime}(d)$.

Step 3: Recall the by definition the EQ coefficients $c_{\lambda, \zeta}^{\mu, d}$ is a homogeneous polynomial in $\Lambda$ of degree $|\lambda|-|\mu|+|\zeta|-d(2 n+2-k)$. We will now compute all coefficients $c_{\lambda, \zeta}^{\mu, d}$ by descending induction on $|\lambda|-|\mu|+|\zeta|-d(2 n+2-k)$ within the induction already occurring on $d$.

Base case: The degree is at most $2 \cdot \operatorname{dim} \operatorname{IG}(k, 2 n+1)-d(2 n+2-k)$. In this case $\lambda=\zeta=(\mathrm{full})$ and $\mu=(0)$. Since $\lambda$ is not included in $\mu$ then Proposition 12.2.1 implies the coefficients $c_{(f u l l),(f u l l)}^{(0), d}$ is known by induction on $d$. This establishes the base case.

Let $c_{\lambda, \zeta}^{\mu, d}$ have polynomial degree less than $2 \cdot \operatorname{dim} \operatorname{IG}(k, 2 n+1)-d(2 n+2-k)$. If $\lambda=\zeta=\mu$ then apply step 2 of this proof. Otherwise, since $c_{\lambda, \zeta}^{\mu, d}=c_{\zeta, \lambda}^{\mu, d}$ then we may assume that $\lambda$ is different from $\mu$. Use equation 12.1 to write $c_{\lambda, \zeta}^{\mu, d}$ as a combination of EQ coefficients of polynomial $|\lambda|-|\mu|+|\zeta|-d(2 n+2-k)+1$ (i.e. one larger) using the first and second terms, and EQ coefficients of degree $d-1$ using the third though sixth terms. The third though sixth terms are known by induction on $d$. The polynomial degree induction, applied to the coefficients appearing in the first through fourth terms of equation 12.1 completes the algorithm. 


\subsection{Multiplication table for $\mathrm{QH}_{T}^{*}(\operatorname{IG}(2,5))$}

$$
\begin{aligned}
& {[X(0)] \star[X(\lambda)]=[X(\lambda)] \text { for all } \lambda \in \Lambda \text {. }} \\
& {[X(1)] \star \quad[X(1)]=2 t_{3}[X(1)]+[X(3,-1)]+2[X(2)]} \\
& {[X(1)] \star \quad[X(2)]=2 t_{2}[X(2)]+[X(2,1)]+[X(3)]} \\
& {[X(1)] \star[X(3,-1)]=\left(t_{1}+t_{3}\right)[X(3,-1)]+[X(3)]} \\
& {[X(1)] \star[X(2,1)]=2\left(t_{2}+t_{3}\right)[X(2,1)]+[X(3,1)]} \\
& {[X(1)] \star[X(3)]=\left(t_{1}+t_{2}\right)[X(3)]+[X(3,1)]+q} \\
& {[X(1)] \star[X(3,1)]=\left(t_{1}+t_{2}+2 t_{3}\right)[X(3,1)]+[X(3,2)]+q[X(1)]} \\
& {[X(1)] \star[X(3,2)]=\left(t_{1}+2 t_{2}+t_{3}\right)[X(3,2)]+q[X(2)]+q[X(3,-1)]} \\
& [X(2)] \star \quad \star X(2)]=2 t_{2}\left(t_{2}-t_{3}\right)[X(2)]+2 t_{2}[X(2,1)]+\left(t_{2}-t_{3}\right)[X(3)]+[X(3,1)] \\
& {[X(2)] \star[X(3,-1)]=\left(t_{1}+t_{2}\right)[X(3)]+q} \\
& {[X(2)] \star \quad[X(2,1)]=2 t_{2}\left(t_{2}+t_{3}\right)[X(2,1)]+\left(t_{2}+t_{3}\right)[X(3,1)]+[X(3,2)]} \\
& {[X(2)] \star[X(3)]=\left(t_{1}+t_{2}\right)\left(t_{2}-t_{3}\right)[X(3)]+\left(t_{1}+t_{2}\right)[X(3,1)]+\left(t_{2}-t_{3}\right) q+q[X(1)]} \\
& {[X(2)] \star[X(3,1)]=\left(t_{1} t_{2}+t_{1} t_{3}+t_{2}^{2}+t_{2} t_{3}\right)[X(3,1)]+\left(t_{1}+t_{2}\right)[X(3,2)]+\left(t_{2}+t_{3}\right) q[X(1)]} \\
& +q[X(3,-1)]+q[X(2)] \\
& {[X(2)] \star[X(3,2)]=2 t_{2}\left(t_{1}+t_{2}\right)[X(3,2)]+\left(t_{1}+t_{2}\right) q[X(3,-1)]+2 t_{2} q[X(2)]+q[X(3)]} \\
& [X(3,-1)] \star \star X(3,-1)]=\left(t_{1}^{2}-t_{3}^{2}\right)[X(3,-1)]-\left(t_{2}+t_{3}\right)[X(3)]+[X(3,1)]-q \\
& {[X(3,-1)] \star[X(2,1)]=\left(t_{1}+t_{2}\right)[X(3,1)]-[X(3,2)]+q[X(1)]} \\
& {[X(3,-1)] \star \quad[X(3)]=\left(t_{1}^{2}-t_{2}^{2}\right)[X(3)]+[X(3,2)]+\left(t_{1}-t_{2}\right) q} \\
& {[X(3,-1)] \star \quad[X(3,1)]=\left(t_{1}^{2}-t_{2}^{2}\right)[X(3,1)]+\left(t_{2}+t_{3}\right)[X(3,2)]+\left(t_{1}-t_{2}\right) q[X(1)]+q[X(2)]} \\
& [X(3,-1)] \star \quad \star X(3,2)]=\left(t_{1}^{2}-t_{3}^{2}\right)[X(3,2)]+\left(t_{1}-t_{3}\right) q[X(2)]+q[X(2,1)] \\
& {[X(2,1)] \star[X(2,1)]=4 t_{2} t_{3}\left(t_{2}+t_{3}\right)[X(2,1)]+2 t_{3}\left(t_{2}+t_{3}\right)[X(3,1)]+2\left(t_{2}+t_{3}\right)[X(3,2)]} \\
& +q[X(3,-1)] \\
& {[X(2,1)] \star[X(3)]=\left(t_{1} t_{2}+t_{1} t_{3}+t_{2}^{2}+t_{2} t_{3}\right)[X(3,1)]+\left(t_{1}-t_{2}\right)[X(3,2)]+\left(t_{2}+t_{3}\right) q[X(1)]} \\
& +q[X(2)] \\
& {[X(2,1)] \star \quad[X(3,1)]=2 t_{3}\left(t_{1} t_{2}+t_{1} t_{3}+t_{2}^{2}+t_{2} t_{3}\right)[X(3,1)]+2 t_{1}\left(t_{2}+t_{3}\right)[X(3,2)]} \\
& +\left(t_{1}+t_{3}\right) q[X(3,-1)]+2 t_{3}\left(t_{2}+t_{3}\right) q[X(1)]+2\left(t_{2}+t_{3}\right) q[X(2)]+q[X(3)] \\
& [X(2,1)] \star \quad \star X(3,2)]=2 t_{2}\left(t_{1} t_{2}+t_{1} t_{3}+t_{2} t_{3}+t_{3}^{2}\right)[X(3,2)]+\left(t_{2}+t_{3}\right)\left(t_{1}+t_{3}\right) q[X(3,-1)] \\
& +2 t_{2}\left(t_{2}+t_{3}\right) q[X(2)]+\left(t_{1}+2 t_{2}+t_{3}\right) q[X(3)]+q^{2} \\
& {[X(3)] \star[X(3)]=\left(t_{1}+t_{2}\right)\left(t_{1} t_{2}-t_{1} t_{3}-t_{2}^{2}+t_{2} t_{3}\right)[X(3)]+\left(t_{1}^{2}-t_{2}^{2}\right)[X(3,1)]+2 t_{2}[X(3,2)]} \\
& +\left(t_{1} t_{2}-t_{1} t_{3}-t_{2}^{2}+t_{2} t_{3}\right) q+\left(t_{1}-t_{2}\right) q[X(1)]+q[X(3,-1)]+q[X(2)] \\
& {[X(3)] \star \quad[X(3,1)]=\left(t_{1}^{2} t_{2}+t_{1}^{2} t_{3}-t_{2}^{3}-t_{2}^{2} t_{3}\right)[X(3,1)]+\left(t_{1}^{2}+t_{2}^{2}+2 t_{2} t_{3}\right)[X(3,2)]} \\
& +\left(t_{1}+t_{3}\right) q[X(3,-1)]+\left(t_{1} t_{2}+t_{1} t_{3}-t_{2}^{2}-t_{2} t_{3}\right) q[X(1)]+\left(t_{1}+t_{2}\right) q[X(2)] \\
& +q[X(2,1)]+q[X(3)] \\
& {[X(3)] \star \quad[X(3,2)]=2 t_{2}\left(t_{1}^{2}-t_{3}^{2}\right)[X(3,2)]+\left(t_{1}^{2}-t_{3}^{2}\right) q[X(3,-1)]} \\
& +2 t_{2}\left(t_{1}-t_{3}\right) q[X(2)]+2 t_{2} q[X(2,1)]+\left(t_{1}-t_{3}\right) q[X(3)]+q[X(3,1)]
\end{aligned}
$$




$$
\begin{aligned}
{[X(3,1)] } & \star[X(3,1)]=2 t_{3}\left(t_{1}^{2} t_{2}+t_{1}^{2} t_{3}-t_{2}^{3}-t_{2}^{2} t_{3}\right)[X(3,1)]+2\left(t_{1}^{2} t_{2}+t_{1}^{2} t_{3}+t_{2}^{2} t_{3}+t_{2} t_{3}^{2}\right)[X(3,2)] \\
& +\left(t_{1}^{2}+2 t_{1} t_{3}+t_{3}^{2}\right) q[X(3,-1)]+2 t_{3}\left(t_{1} t_{2}+t_{1} t_{3}-t_{2}^{2}-t_{2} t_{3}\right) q[X(1)] \\
& +2 t_{1}\left(t_{2}+t_{3}\right) q[X(2)]+2\left(t_{2}+t_{3}\right) q[X(2,1)]+\left(2 t_{1}+t_{2}+t_{3}\right) q[X(3)]+q[X(3,1)]+q^{2} \\
{[X(3,1)] } & \star[X(3,2)]=2 t_{2}\left(t_{1}^{2} t_{2}+t_{1}^{2} t_{3}-t_{2} t_{3}^{2}-t_{3}^{3}\right)[X(3,2)]+\left(t_{2}+t_{3}\right)\left(t_{1}^{2}-t_{3}^{2}\right) q[X(3,-1)] \\
& +2 t_{2}\left(t_{1} t_{2}+t_{1} t_{3}-t_{2} t_{3}-t_{3}^{2}\right) q[X(2)]+2 t_{2}\left(t_{2}+t_{3}\right) q[X(2,1)] \\
& +\left(t_{1}+2 t_{2}+t_{3}\right)\left(t_{1}-t_{3}\right) q[X(3)]+\left(t_{1}+2 t_{2}+t_{3}\right) q[X(3,1)]+\left(t_{1}-t_{3}\right) q^{2}+q^{2}[X(1)] \\
\hline[X(3,2)] & \star[X(3,2)]=2 t_{2}\left(t_{1}^{2} t_{2}^{2}-t_{1}^{2} t_{3}^{2}-t_{2}^{2} t_{3}^{2}+t_{3}^{4}\right)[X(3,2)]+\left(t_{1}^{2} t_{2}^{2}-t_{1}^{2} t_{3}^{2}-t_{2}^{2} t_{3}^{2}+t_{3}^{4}\right) q[X(3,-1)] \\
& +2 t_{2}\left(t_{1} t_{2}^{2}-t_{1} t_{3}^{2}-t_{2}^{2} t_{3}+t_{3}^{3}\right) q[X(2)]+2 t_{2}\left(t_{2}^{2}-t_{3}^{2}\right) q[X(2,1)] \\
& +\left(t_{1}+2 t_{2}+t_{3}\right)\left(t_{1} t_{2}-t_{1} t_{3}-t_{2} t_{3}+t_{3}^{2}\right) q[X(3)]+\left(t_{1}^{2}+2 t_{1} t_{2}+2 t_{2}^{2}-t_{3}^{2}\right) q[X(3,1)] \\
& +\left(t_{1} t_{2}-t_{1} t_{3}-t_{2} t_{3}+t_{3}^{2}\right) q^{2}+\left(t_{1}+t_{2}\right) q^{2}[X(1)]+q^{2}[X(2)]
\end{aligned}
$$




\section{Appendix A}

\section{The first Chern class of the tangent bundle $c_{1}\left(T_{\mathrm{IG}}\right)$.}

In this appendix we will calculate the first Chern class of the tangent bundle $c_{1}\left(T_{\mathrm{IG}}\right)$. The calculation is stated next.

Theorem A.0.1. The first Chern class of the tangent bundle is

$$
c_{1}\left(T_{\mathrm{IG}}\right)=(2 n+2-k)[X(D i v)]+k t_{1}+\sum_{i=2}^{k+1}(2 n+2-k) t_{i} .
$$

Before we prove this we need to prove a few Lemmas. We use Mihai's calculation a for $\mathrm{H}_{T}^{*}(\operatorname{IF}(1,2, \cdots, n+1 ; 2 n+1))$ in [47]. Let $\pi_{k}: \operatorname{IF}(1,2, \cdots, n+1 ; 2 n+1) \rightarrow$ IG and $\pi_{k}^{\prime}: \operatorname{IF}(1,2, \cdots, n+1 ; 2 n+2) \rightarrow \mathrm{IG}^{\text {even }}$ be the $k$ th projection maps. Let $\left[S_{D i v_{1}}\right], \cdots,\left[S_{D i v_{n}}\right],\left[S_{D i v_{n+1}}\right]$ be the divisor classes of $\mathrm{H}_{T}^{*}(\operatorname{IF}(1,2, \cdots, n+1 ; 2 n+1))$ where

$$
\begin{aligned}
S_{D i v_{1}} & =S_{\overline{3} \overline{4} \overline{4} \cdots \overline{n+1} 1} \\
& \vdots \\
S_{D i v_{i}} & =S_{\overline{2} \cdots \bar{i} \overline{i+2} \overline{i+1} \overline{i+3} \cdots \overline{n+1} 1} \\
& \vdots \\
S_{D i v_{n-1}} & =S_{\overline{2} \cdots \overline{n-1} \overline{n+1} \bar{n} 1} \\
S_{D i v_{n}} & =S_{\overline{2} \cdots \bar{n}(n+1) 1} \\
S_{D i v_{n+1}} & =S_{\overline{2} \cdots \bar{n} 1 \overline{n+1}}
\end{aligned}
$$

Recall $w_{0}=\overline{23} \cdots \overline{n+1} 1$ in the Weyl group $W$.

Lemma A.0.2. We have the following

$$
\begin{aligned}
\pi_{k}^{*}([X(\text { Div })]) & =\left[S_{D i v_{k}}\right] \text { for } 1 \leq k \leq n-1 \\
\pi_{n}^{*}([X(\text { Div })]) & =\left[S_{D i v_{n}}\right]+\left[S_{D i v_{n+1}}\right] \\
\pi_{n+1}^{*}([X(\text { Div })]) & =\left[S_{D i v_{n+1}}\right] .
\end{aligned}
$$


Proof. We have the following diagram

$$
\begin{aligned}
& \mathrm{IF}(1,2, \cdots, n+1 ; 2 n+1) \stackrel{\iota^{\prime}}{\longrightarrow} \operatorname{IF}(1,2, \cdots, n+1 ; 2 n+2) \\
& \downarrow \pi_{k} \quad \downarrow \pi_{k}^{\prime} \\
& \mathrm{IG} \stackrel{\iota}{\longrightarrow} \mathrm{IG}^{\text {even }}
\end{aligned}
$$

where $\pi_{k}$ and $\pi_{k}^{\prime}$ are the $k$ th projective maps.

Let $F_{\bullet}$ be an isotropic flag, $\Sigma_{w}\left(F_{\bullet}\right)$ a Schubert subvariety of $\operatorname{IF}(1,2, \cdots, n+$ $1 ; 2 n+2)$, and $\left[\Sigma_{w}\right]$ the associated Schubert class where $w$ is an element of the Weyl group $W$. We know that $\operatorname{IF}(1,2, \cdots, n+1 ; 2 n+1)$ is isomorphic to the Schubert subvariety $\Sigma_{w_{0}}\left(E_{\bullet}\right)$ of $\operatorname{IF}(1,2, \cdots, n+1 ; 2 n+2)$ where $E_{\bullet}$ is an isotropic flag which we may assume to be in general position with respect to $F_{\bullet}$. Then it follows that the $k$ th divisor $\Sigma_{D i v_{k}}\left(F_{\bullet}\right)$ and $\Sigma_{w_{0}}\left(E_{\bullet}\right)$ meet transversally, hence we can compute the restriction $\left(\iota^{\prime}\right)^{*}\left[\Sigma_{D i v_{k}}\right]$ by computing the class of the intersection $\Sigma_{D i v_{k}} \cup \Sigma_{w_{0}}$ in $\operatorname{IF}(1,2, \cdots, n+1 ; 2 n+1)$ using the Chevalley rules for $\operatorname{IF}(1,2, \cdots, n+1 ; 2 n+2)$.

By the Chevalley formula we have

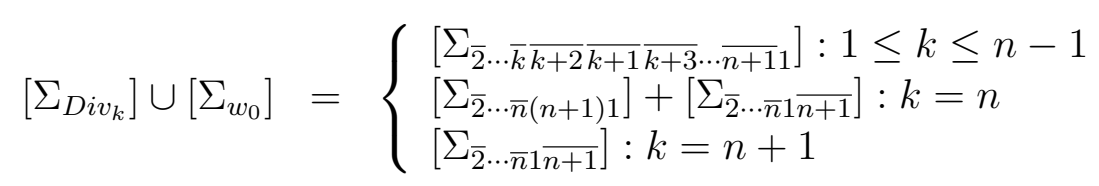

Denote by $\left[S_{w}\right]$ the cohomology class associated to the Schubert variety $S_{w}\left(F_{\bullet}\right) \subset$ $\operatorname{IF}(1,2, \cdots, n+1 ; 2 n+1)$ where $w$ is in the Weyl group $W$ such that $w(j) \neq \overline{1}$ for all $1 \leq j \leq n+1$ and $F_{\bullet}$ is an isotropic flag of $\mathbb{C}^{2 n+1}$ (recall $S_{w}=\Sigma_{w}$ when $\left.\Sigma_{w} \subset \operatorname{IF}(1,2, \cdots, n+1 ; 2 n+2)\right)$.

Incidence conditions give $\iota_{*}^{\prime}\left[S_{w}\right]=\left[\Sigma_{w}\right]$ for all $w \in W$ such that $w(j) \neq \overline{1}$ for all $1 \leq j \leq n+1$.

Now we have

$$
\begin{aligned}
& \left.\iota_{*}^{\prime} \iota^{\prime *}\left[\Sigma_{D i v_{k}}\right)\right]=\iota_{*}^{\prime}\left([\mathrm{IF}(2 n+1)] \cup \iota^{\prime *}\left[\Sigma_{D i v_{k}}\right]\right) \\
& \left.=\left(\iota_{*}^{\prime}[\operatorname{IF}(2 n+1)]\right) \cup\left[\Sigma_{D i v_{k}}\right)\right] \text { by the projection formula } \\
& =\left[\Sigma_{\overline{2} \cdots \overline{n+1} 1}\right] \cup\left[\Sigma_{D i v_{k}}\right]
\end{aligned}
$$

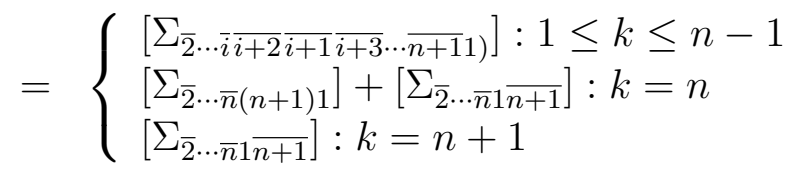

$$
\begin{aligned}
& \text { Therefore, }\left(\iota^{\prime}\right)^{*}\left[\Sigma_{D i v_{k}}\right]=\left\{\begin{array}{l}
{\left[S_{D i v_{k}}\right]: 1 \leq k \leq n-1} \\
{\left[S_{D i v_{n}}\right]+\left[S_{D i v_{n+1}}\right]: k=n} \\
{\left[S_{D i v_{n+1}}\right]: k=n+1}
\end{array}\right.
\end{aligned}
$$


Recall $\iota^{*}([Y(D i v)])=[X(D i v)]$. Then we have

$$
\begin{aligned}
\pi_{k}^{*}([X(\text { Div })])= & \pi_{k}^{*}\left(\iota^{*}([Y(\text { Div })])\right) \\
= & \iota^{\prime *} \pi_{k}^{\prime *}([Y(\text { Div })]) \\
= & \left(\iota^{\prime}\right)^{*}\left[\Sigma_{D i v_{k}}\right] \\
& =\left\{\begin{array}{l}
{\left[S_{D i v_{k}}\right]: 1 \leq k \leq n-1} \\
{\left[S_{D i v_{n}}\right]+\left[S_{D i v_{n+1}}\right]: k=n} \\
{\left[S_{D i v_{n+1}}\right]: k=n+1}
\end{array}\right.
\end{aligned}
$$

Lemma A.0.3. The first Chern class of the tangent bundle localized at the point $w_{0}=(\overline{k+1}<\bar{k}<\cdots<\overline{2})$ is

$$
\left.c_{1}\left(T_{\mathrm{IG}}\right)\right|_{w_{0}}=k t_{1}+\sum_{i=2}^{k+1}(2 n+2-k) t_{i} .
$$

Proof. The Schubert cell around the $T$-fixed point $w_{0}$ is $C\left(w_{0}\right)$. Let $t \in T$. Then 


$$
\begin{aligned}
& t \cdot C\left(w_{0}\right)=\left(\begin{array}{cccccc}
t_{1} * & t_{1} * & t_{1} * & \cdots & t_{1} * & t_{1} * \\
t_{2} * & \bullet & \bullet & \cdots & \bullet & \bullet \\
t_{3} * & t_{3} * & \bullet & \cdots & \bullet & \bullet \\
\vdots & \vdots & \vdots & \cdots & \vdots & \vdots \\
t_{k} * & t_{k} * & t_{k} * & \cdots & t_{k} * & \bullet \\
t_{k+1} * & t_{k+1} * & t_{k+1} * & \cdots & t_{k+1} * & t_{k+1} * \\
\vdots & \vdots & \vdots & \cdots & \vdots & \vdots \\
t_{k+2}^{-1} * & t_{k+2}^{-1} & t_{k+2}^{-1} * & \cdots & t_{k+2}^{-1} * & t_{k+2}^{-1} \\
0 & 0 & 0 & \cdots & 0 & t_{k+1}^{-1} * \\
0 & 0 & 0 & \cdots & t_{k}^{-1} & 0 \\
\vdots & \vdots & \vdots & \cdots & \vdots & \vdots \\
0 & 0 & t_{4}^{-1} & \cdots & 0 & 0 \\
0 & t_{3}^{-1} & 0 & \cdots & 0 & 0 \\
t_{2}^{-1} & 0 & 0 & \cdots & 0 & 0
\end{array}\right) \\
& =\left(\begin{array}{cccccc}
t_{2} t_{1} * & t_{3} t_{1} * & t_{4} t_{1} * & \cdots & t_{k} t_{1} * & t_{k+1} t_{1} * \\
t_{2} t_{2} * & \bullet & \bullet & \cdots & \bullet & \bullet \\
t_{2} t_{3} * & t_{3} t_{3} * & \bullet & \cdots & \bullet & \bullet \\
\vdots & \vdots & \vdots & \cdots & \vdots & \vdots \\
t_{2} t_{k} * & t_{3} t_{k} * & t_{4} t_{k} * & \cdots & t_{k} t_{k} * & \bullet \\
t_{2} t_{k+1} * & t_{3} t_{k+1} * & t_{4} t_{k+1} * & \cdots & t_{k} t_{k+1} * & t_{1}^{-1} t_{k+1} * \\
\vdots & \vdots & \vdots & \cdots & \vdots & \vdots \\
t_{2} t_{k+1}^{-1} * & t_{3} t_{k+1}^{-1} * & t_{4} t_{k+1}^{-1} * & \cdots & t_{k} t_{k+1}^{-1} * & t_{1}^{-1} t_{k+1}^{-1} * \\
0 & 0 & 0 & \cdots & 1 & 0 \\
\vdots & \vdots & \vdots & \cdots & \vdots & \vdots \\
0 & 0 & 1 & \cdots & 0 & 0 \\
0 & 1 & 0 & \cdots & 0 & 0 \\
1 & 0 & 0 & \cdots & 0 & 0
\end{array}\right)
\end{aligned}
$$

Writing the weights in additive notation and adding them together yields

$$
k t_{1}+\sum_{i=2}^{k+1}(2 n+2-k) t_{i}
$$

The result follows.

Lemma A.0.4. The localizations of the divisor class $[X($ Div $)]$ at the Schubert point $X(i d)=(1<2<\cdots<k)$ and $w_{0}$ are

$$
\left.[X(\text { Div })]\right|_{X(i d)}=-t_{1}-2\left(t_{2}+t_{2}+\cdots+t_{k}\right)-t_{k+1} \text { and }\left.[X(\text { Div })]\right|_{w_{0}}=0 .
$$


Proof. Mihai gives the following in [47, Prop. 5.3.33].

For $1 \leq k \leq n-1$ we have $\left.\left[S_{D i v_{k}}\right]\right|_{w}=w_{0}\left(t_{1}+\cdots+t_{k}\right)-w\left(t_{1}+\cdots+t_{k}\right)$

$$
\begin{aligned}
{\left.\left[S_{D i v_{n}}\right]\right|_{w} } & =w_{0}\left(t_{1}+\cdots+t_{n+1}\right)-w\left(t_{1}+\cdots+t_{n+1}\right) \\
{\left.\left[S_{D i v_{n+1}}\right]\right|_{w} } & =w_{0}\left(t_{n+1}\right)-w\left(t_{n+1}\right)
\end{aligned}
$$

Then we have

For $1 \leq k \leq n-1$ we have $\left.[X(D i v)]\right|_{w_{0}}=\left.\pi_{k}^{*}\left(\left[S_{D i v_{k}}\right]\right)\right|_{w_{0}}=\left.\left[S_{D i v_{k}}\right]\right|_{w_{0}}=0$

$$
\text { and }\left.[X(D i v)]\right|_{w_{0}}=\left.\pi_{n}^{*}\left(\left[S_{D i v_{n}}\right]\right)\right|_{w_{0}}=\left.\left[S_{D i v_{n}}\right]\right|_{w_{0}}+\left.\left[S_{D i v_{n+1}}\right]\right|_{w_{0}}=0
$$

and

For $1 \leq k \leq n-1$ we have $\left.[X(D i v)]\right|_{X(i d)}=\left.\pi_{k}^{*}\left(\left[S_{D i v_{k}}\right]\right)\right|_{X(i d)}=\left.\left[S_{D i v_{k}}\right]\right|_{X(i d)}$

$$
\begin{aligned}
& =-t_{1}-2\left(t_{2}+t_{2}+\cdots+t_{k}\right)-t_{k+1} \\
\text { and }\left.[X(D i v)]\right|_{X(i d)} & =\left.\pi_{n}^{*}\left(\left[S_{D i v_{n}}\right]\right)\right|_{X(i d)}=\left.\left[S_{D i v_{n}}\right]\right|_{X(i d)}+\left.\left[S_{D i v_{n+1}}\right]\right|_{X(i d)} \\
& =-t_{1}-2\left(t_{2}+t_{2}+\cdots+t_{n}\right)-t_{n+1}
\end{aligned}
$$

The result follows.

Since $\left.[X(D i v)]\right|_{w_{0}}=0$ it follows

$$
c_{1}\left(T_{\mathrm{IG}}\right)=A[X(D i v)]+k t_{1}+\sum_{i=2}^{k+1}(2 n+2-k) t_{i}
$$

for some $A \in \mathrm{H}_{T}^{*}(p t)$.

Then we have

$$
\left.c_{1}\left(T_{\mathrm{IG}}\right)\right|_{X(i d)}=-A\left(t_{1}+2\left(t_{2}+\cdots+t_{k}\right)+t_{k+1}\right)+k t_{1}+\sum_{i=2}^{k+1}(2 n+2-k) t_{i}
$$

Lemma A.0.5. The first Chern class of the tangent bundle localized at the Schubert point $X(i d)=(1<2<\cdots<k)$ is

$$
\left.c_{1}\left(T_{\mathrm{IG}}\right)\right|_{X(i d)}=-(2 n+2-2 k) t_{1}-\sum_{i=2}^{k}(2 n+2-k) t_{i} .
$$

Proof. The Schubert cell around the $T$-fixed point $X(i d)=(1<2<\cdots<k)$ is $C(i d)$. Let $t \in T$. Then 


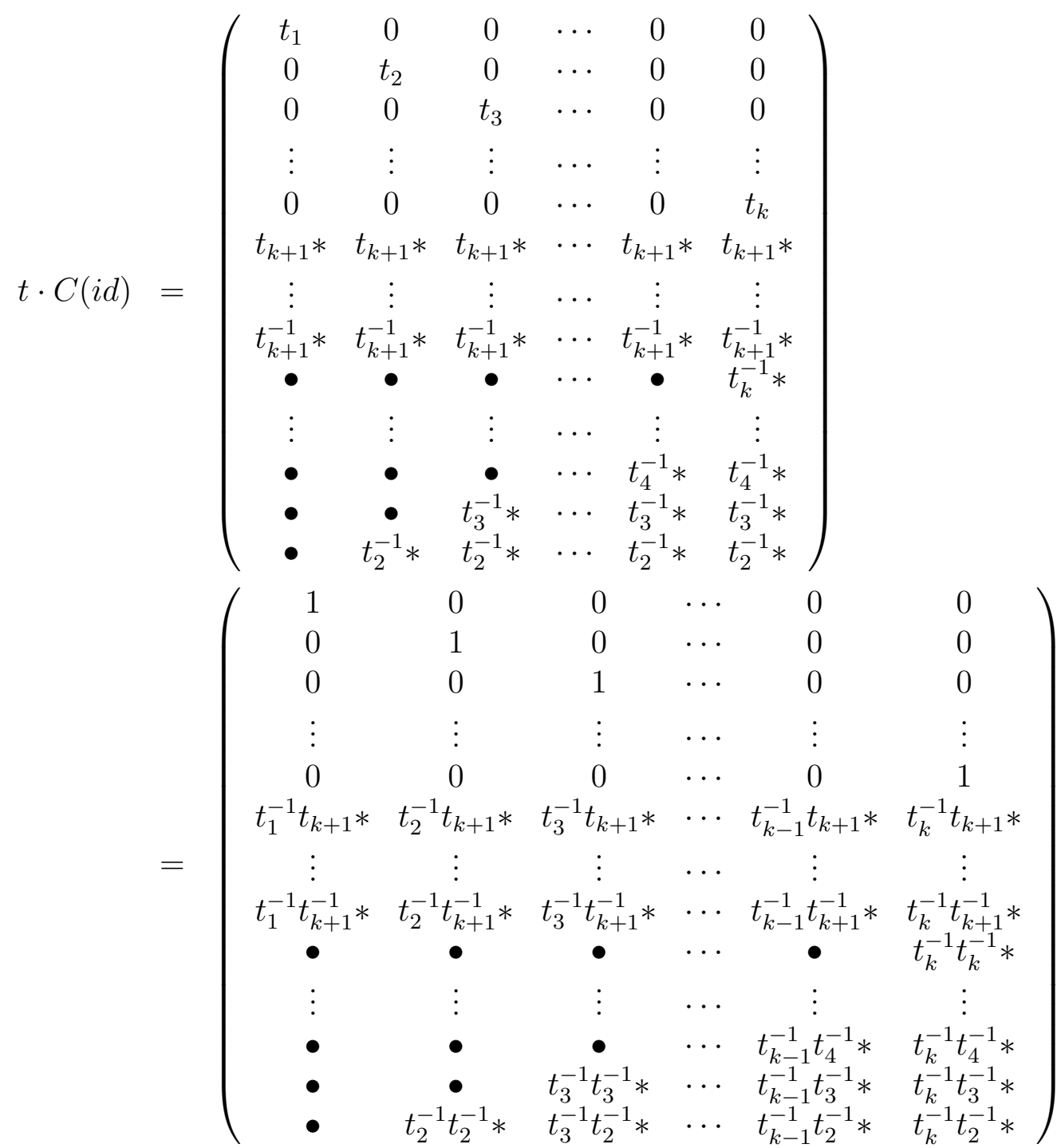

Writing the weights in additive notation and add them together yields

$$
-(2 n+2-2 k) t_{1}-\sum_{i=2}^{k}(2 n+2-k) t_{i}
$$

The result follows.

Proof of Theorem A.0.1. The Lemmas imply a nontrivial identity which is

$$
\begin{aligned}
-(2 n+2-2 k) t_{1}-\sum_{i=2}^{k}(2 n+2-k) t_{i} & =-A\left(t_{1}+2\left(t_{2}+\cdots+t_{k}\right)+t_{k+1}\right) \\
& +k t_{1}+\sum_{i=2}^{k+1}(2 n+2-k) t_{i} .
\end{aligned}
$$


Thus, $A=2 n+2-k$. The result follows. 


\section{Appendix B}

\section{Lines}

The purpose of this Appendix is state that lines in $\operatorname{IG}(k, 2 n+1)$ are also lines in $\operatorname{Gr}(k, 2 n+2)$.

Lemma B.0.1. (a) For any two points $x, y \in \operatorname{IG}(k, 2 n+1)$ there exists at most one line $L$ such that $x, y \in L$. (b) Let $L$ be a line in $\operatorname{IG}(k, 2 n+1)$ passing through two T-fixed points. Then $L$ is T-stable.

Proof. First recall that $\iota: \operatorname{IG}(k, 2 n+1) \hookrightarrow \mathrm{IG}(k, 2 n+2)$ is the natural embedding and $\iota^{*}[Y(1)]=[X(1)]($ see $[55])$. That is, if $L$ is a line in $\operatorname{IG}(k, 2 n+1)$ then $\iota(L)$ is a line in $\mathrm{IG}(k, 2 n+2)$.

Let $j: \operatorname{IG}(k, 2 n+2) \hookrightarrow \operatorname{Gr}(k, 2 n+2)$ be the natural embedding. Let $S_{\mathrm{IG}}$ be the tautological bundle over $\mathrm{IG}(k, 2 n+2)$ and $S_{\mathrm{Gr}}$ be the tautological bundle over $\operatorname{Gr}(k, 2 n+2)$. Since $[Y(1)]$ is the only generator of $H^{2}(\operatorname{IG}(k, 2 n+2))$ then the first Chern class is

$$
c_{1}\left(S_{\mathrm{IG}}^{*}\right)=a[Y(1)]
$$

for some integer $a$. Define $Y_{C}$ to be the Schubert curve of $\operatorname{IG}(k, 2 n+2)$. We want to show that $a=1$ by showing

$$
\int_{\mathrm{IG}(k, 2 n+2)} c_{1}\left(S_{\mathrm{IG}}^{*}\right) \cap\left[Y_{C}\right]=1 .
$$

In coordinates the open cell of $Y_{C}$ is

$$
Y(1<2<3<\cdots<k-1<k+1)^{\circ}=\left(\begin{array}{cccccc}
1 & 0 & 0 & \cdots & 0 & 0 \\
0 & 1 & 0 & \cdots & 0 & 0 \\
0 & 0 & 1 & \cdots & 0 & 0 \\
\vdots & \vdots & \vdots & \ddots & \vdots & \vdots \\
0 & 0 & 0 & \cdots & 1 & 0 \\
0 & 0 & 0 & \cdots & 0 & * \\
0 & 0 & 0 & \cdots & 0 & 1 \\
0 & 0 & 0 & \cdots & 0 & 0 \\
\vdots & \vdots & \vdots & \vdots & \vdots & \vdots \\
0 & 0 & 0 & \cdots & 0 & 0
\end{array}\right) .
$$


Since the open cell of $Y_{C}$ has no dependence on the symplectic form $\omega$ then we can conclude that $j_{*}\left[Y_{C}\right]$ is the Schubert curve for $\operatorname{Gr}(k, 2 n+2)$. We can now use the projection formula to compute

$$
\begin{aligned}
\int_{\mathrm{IG}(k, 2 n+2)} c_{1}\left(S_{\mathrm{IG}}^{*}\right) \cap\left[Y_{C}\right] & =\int_{\mathrm{IG}(k, 2 n+2)} c_{1}\left(j^{*} S_{\mathrm{Gr}}^{*}\right) \cap\left[Y_{C}\right] \\
& =\int_{\mathrm{Gr}(k, 2 n+2)} c_{1}\left(S_{\mathrm{Gr}}^{*}\right) \cap j_{*}\left[Y_{C}\right] \\
& =1 .
\end{aligned}
$$

Therefore, $c_{1}\left(S_{\mathrm{IG}}^{*}\right)=[Y(1)]$. We have shown if $L$ is a line in $\operatorname{IG}(k, 2 n+2)$ then $j(L)$ is a line $\operatorname{Gr}(k, 2 n+2)$. We have also shown that $j \circ \iota: \operatorname{IG}(k, 2 n+1) \hookrightarrow \operatorname{Gr}(k, 2 n+2)$ is the natural embedding. We have shown that if $L$ is a line in $\operatorname{IG}(k, 2 n+1)$ then $(j \circ \iota)(L)$ is a line in $\operatorname{Gr}(k, 2 n+2)$.

It is know for any two points $x, y \in \operatorname{Gr}(k, 2 n+2)$ there exists at most one line $L$ such that $x, y \in L$. It is also known if $L$ is a line in $\operatorname{Gr}(k, 2 n+2)$ passing through two $T_{\mathrm{Gr}}$-fixed points then $T_{\mathrm{Gr}}$-stable where it is $T_{\mathrm{Gr}}=\left(t_{1}, t_{2}, \cdots, t_{2 n+2}\right)$. This completes the proof since $T$ is a subgroup of $T_{\mathrm{Gr}}$. 


\section{Bibliography}

[1] Quantum cohomology at the mittag-leffler institute, Appunti dei Corsi Tenuti da Docenti della Scuola. [Notes of Courses Given by Teachers at the School], Scuola Normale Superiore, Pisa, pp. vi+163.

[2] Dave Anderson, Introduction to equivariant cohomology in algebraic geometry, Contributions to algebraic geometry, EMS Ser. Congr. Rep., Eur. Math. Soc., Zürich, 2012, pp. 71-92. MR 2976939

[3] Alexander Astashkevich and Vladimir Sadov, Quantum cohomology of partial flag manifolds $F_{n_{1} \cdots n_{k}}$, Comm. Math. Phys. 170 (1995), no. 3, 503-528. MR 1337131

[4] Nantel Bergeron and Frank Sottile, A Pieri-type formula for isotropic flag manifolds, Trans. Amer. Math. Soc. 354 (2002), no. 7, 2659-2705. MR 1895198

[5] Aaron Bertram, Quantum Schubert calculus, Adv. Math. 128 (1997), no. 2, 289305. MR 1454400

[6] A. Bial ynicki Birula, Some theorems on actions of algebraic groups, Ann. of Math. (2) 98 (1973), 480-497. MR 0366940

[7] Michel Brion, Lectures on the geometry of flag varieties, Topics in cohomological studies of algebraic varieties, Trends Math., Birkhäuser, Basel, 2005, pp. 33-85. MR 2143072

[8] Anders Buch, Pierre-Emmanuel Chaput, Leonardo C. Mihalcea, and Nicolas Perrin, A chevalley formula for the equivariant quantum $k$-theory of cominuscule varieties, https://arxiv.org/pdf/1604.07500.pdf.

[9] _ Finiteness of cominuscule quantum K-theory, Annales Sci. de L'École Normale Supérieure (2013), no. 46.

[10] Anders Skovsted Buch, Quantum cohomology of Grassmannians, Compositio Math. 137 (2003), no. 2, 227-235. MR 1985005

[11] Q Quantum cohomology of partial flag manifolds, Trans. Amer. Math. Soc. 357 (2005), no. 2, 443-458. MR 2095617 
[12] Anders Skovsted Buch, Andrew Kresch, Kevin Purbhoo, and Harry Tamvakis, The puzzle conjecture for the cohomology of two-step flag manifolds, J. Algebraic Combin. 44 (2016), no. 4, 973-1007. MR 3566227

[13] Anders Skovsted Buch, Andrew Kresch, and Harry Tamvakis, Quantum Pieri rules for isotropic Grassmannians, Invent. Math. 178 (2009), no. 2, 345-405. MR 2545685

[14] _ A Giambelli formula for even orthogonal Grassmannians, J. Reine Angew. Math. 708 (2015), 17-48. MR 3420327

[15] Buch, A. and Kresch, A. and Tamvakis, H., Quantum Giambelli formulas for isotropic Grassmannians, Math. Ann. 354, no. 3, 801-812.

[16] Buch, Anders S. and Mihalcea, Leonardo C., Curve neighborhoods of Schubert varieties, J. Differential Geom. 99 (2015), no. 2, 255-283. MR 3302040

[17] Linda Chen, Quantum cohomology of flag manifolds, Adv. Math. 174 (2003), no. 1, 1-34. MR 1959889

[18] C. Chevalley, Sur les décompositions cellulaires des espaces $G / B$, Algebraic groups and their generalizations: classical methods (University Park, PA, 1991), Proc. Sympos. Pure Math., vol. 56, Amer. Math. Soc., Providence, RI, 1994, With a foreword by Armand Borel, pp. 1-23. MR 1278698

[19] Alessio Corti and Miles Reid, Weighted Grassmannians, Algebraic geometry, de Gruyter, Berlin, 2002, pp. 141-163. MR 1954062

[20] Fukukawa, Y. and Ishida, H. and Masuda, M., The cohomology ring of the GKM graph of a flag manifold of classical type, Kyoto J. Math. 54, no. 3, 653-677.

[21] W. Fulton and R. Pandharipande, Notes on stable maps and quantum cohomology, Algebraic geometry - Santa Cruz 1995, Proc. Sympos. Pure Math., vol. 62, Amer. Math. Soc., Providence, RI, 1997, pp. 45-96. MR 1492534

[22] W. Fulton and C. Woodward, On the quantum product of Schubert classes, J. Algebraic Geom. 13 (2004), no. 4, 641-661. MR 2072765

[23] William Fulton, Young tableaux, London Mathematical Society Student Texts, vol. 35, Cambridge University Press, Cambridge, 1997, With applications to representation theory and geometry. MR 1464693

[24] _ Intersection theory, second ed., Ergebnisse der Mathematik und ihrer Grenzgebiete. 3. Folge. A Series of Modern Surveys in Mathematics [Results in Mathematics and Related Areas. 3rd Series. A Series of Modern Surveys in Mathematics], vol. 2, Springer-Verlag, Berlin, 1998. MR 1644323 
[25] William Fulton and Joe Harris, Representation theory, Graduate Texts in Mathematics, vol. 129, Springer-Verlag, New York, 1991, A first course, Readings in Mathematics. MR 1153249

[26] I. M. Gel'fand and A. V. Zelevinskiü, Models of representations of classical groups and their hidden symmetries, Funktsional. Anal. i Prilozhen. 18 (1984), no. 3, 14-31. MR 757246

[27] Alexander Givental and Bumsig Kim, Quantum cohomology of flag manifolds and Toda lattices, Comm. Math. Phys. 168 (1995), no. 3, 609-641. MR 1328256

[28] Alexander B. Givental, Equivariant Gromov-Witten invariants, Internat. Math. Res. Notices (1996), no. 13, 613-663. MR 1408320

[29] M. Goresky, R. Kottwitz, and R. MacPherson.

[30] T. Graber, Enumerative geometry of hyperelliptic plane curves, J. Algebraic Geom. 10, no. 4, 725-755.

[31] Robin Hartshorne, Algebraic geometry, Springer-Verlag, New York-Heidelberg, 1977, Graduate Texts in Mathematics, No. 52. MR 0463157

[32] James E. Humphreys, Linear algebraic groups, Springer-Verlag, New YorkHeidelberg, 1975, Graduate Texts in Mathematics, No. 21. MR 0396773

[33] _ Reflection groups and Coxeter groups, Cambridge Studies in Advanced Mathematics, vol. 29, Cambridge University Press, Cambridge, 1990. MR 1066460

[34] B. Kim and R. Pandharipande, The connectedness of the moduli space of maps to homogeneous spaces, Symplectic geometry and mirror symmetry (Seoul, 2000), World Sci. Publ., River Edge, NJ, 2001, pp. 187-201. MR 1882330

[35] Bumsig Kim, On equivariant quantum cohomology, Internat. Math. Res. Notices (1996), no. 17, 841-851. MR 1420551

[36] Steven L. Kleiman, The transversality of a general translate, Compositio Math. 28 (1974), 287-297. MR 0360616

[37] Allen Knutson and Terence Tao, Puzzles and (equivariant) cohomology of Grassmannians, Duke Math. J. 119 (2003), no. 2, 221-260. MR 1997946

[38] M. Kontsevich and Yu. Manin, Gromov-Witten classes, quantum cohomology, and enumerative geometry, Comm. Math. Phys. 164 (1994), no. 3, 525-562. MR 1291244

[39] Maxim Kontsevich, Enumeration of rational curves via torus actions, The moduli space of curves (Texel Island, 1994), Progr. Math., vol. 129, Birkhäuser Boston, Boston, MA, 1995, pp. 335-368. MR 1363062 
[40] B. Kostant and S. Kumar, T-equivariant $K$-theory of generalized flag varieties, Proc. Nat. Acad. Sci. U.S.A. 84, no. 13, 4351-4354.

[41] Andrew Kresch and Harry Tamvakis, Quantum cohomology of the Lagrangian Grassmannian, J. Algebraic Geom. 12 (2003), no. 4, 777-810. MR 1993764

[42] _ Quantum cohomology of orthogonal Grassmannians, Compos. Math. 140 (2004), no. 2, 482-500. MR 2027200

[43] Changzheng Li and Leonardo C. Mihalcea, K-theoretic Gromov-Witten invariants of lines in homogeneous spaces, Int. Math. Res. Not. IMRN (2014), no. 17, 4625-4664. MR 3257546

[44] A. Mare and L. Mihalcea, An affine deformation of the quantum cohomology ring of flag manifolds and periodic toda lattice, https://arxiv.org/pdf/1409.3587.pdf.

[45] H. Matsumura, Commutative ring theory, Cambridge Studies in Advanced Mathematics, vol. 8, Cambridge University Press, Cambridge, Translated from the Japanese by M. Reid.

[46] Ion Alexandru Mihai, Odd symplectic flag manifolds, Transform. Groups 12 (2007), no. 3, 573-599. MR 2356323

[47] Mihai, I., Variétés de drapeaux symplectiques impaires, Ph.D. thesis, Institut Fourier, 2005.

[48] L. Mihalcea and R. Shifler, Equivariant quantum cohomology of the odd symplectic grassmannian, In Preparation.

[49] L. C. Mihalcea, Positivity in equivariant quantum schubert calculus, Amer. J. Math. 128, no. 3, 787-803.

[50] Leonardo Mihalcea, Equivariant quantum Schubert calculus, Adv. Math. 203 (2006), no. 1, 1-33. MR 2231042

[51] Leonardo Constantin Mihalcea, On equivariant quantum cohomology of homogeneous spaces: Chevalley formulae and algorithms, Duke Math. J. 140 (2007), no. 2, 321-350. MR 2359822

[52] Alexander I. Molev and Bruce E. Sagan, A Littlewood-Richardson rule for factorial Schur functions, Trans. Amer. Math. Soc. 351 (1999), no. 11, 4429-4443. MR 1621694

[53] David Mumford, The red book of varieties and schemes, expanded ed., Lecture Notes in Mathematics, vol. 1358, Springer-Verlag, Berlin, 1999, Includes the Michigan lectures (1974) on curves and their Jacobians, With contributions by Enrico Arbarello. MR 1748380 
[54] Andrei Okounkov, Quantum immanants and higher Capelli identities, Transform. Groups 1 (1996), no. 1-2, 99-126. MR 1390752

[55] Pech, C., Cohomologie quantique des grassmanniennes symplectiques impaire, Ph.D. thesis, Université de Grenoble.

[56] _ Quantum cohomology of the odd symplectic Grassmannian of lines, J. Algebra 375, 188-215.

[57] Robert A. Proctor, Odd symplectic groups and combinatorics, Lie algebras and related topics (Windsor, Ont., 1984), CMS Conf. Proc., vol. 5, Amer. Math. Soc., Providence, RI, 1986, pp. 357-360. MR 832210

[58] _ Odd symplectic groups, Invent. Math. 92 (1988), no. 2, 307-332. MR 936084

[59] Yongbin Ruan and Gang Tian, A mathematical theory of quantum cohomology, Math. Res. Lett. 1 (1994), no. 2, 269-278. MR 1266766

[60] H. Schubert, Kalkül der abzählenden geometrie, Teubner, Leipzig, 1874.

[61] Frank Sottile, Pieri-type formulas for maximal isotropic Grassmannians via triple intersections, Colloq. Math. 82 (1999), no. 1, 49-63. MR 1736034

[62] Harry Tamvakis, Quantum cohomology of isotropic Grassmannians, Geometric methods in algebra and number theory, Progr. Math., vol. 235, Birkhäuser Boston, Boston, MA, 2005, pp. 311-338. MR 2166090

[63] Harry Tamvakis and Elizabeth Wilson, Double theta polynomials and equivariant Giambelli formulas, Math. Proc. Cambridge Philos. Soc. 160 (2016), no. 2, 353377. MR 3458957

[64] Jesper Funch Thomsen, Irreducibility of $\bar{M}_{0, n}(G / P, \beta)$, Internat. J. Math. 9 (1998), no. 3, 367-376. MR 1625369 